\title{
Quasi-Relativistic Calculation of EPR
}

\section{g-Tensors with Derivatives of the Decoupling \\ Transformation, Gauge-Including Atomic Orbitals, and Magnetic Balance}

\author{
Yannick J. Franzke*,† and Jason M. Yu*,† \\ $\dagger$ Fachbereich Chemie, Philipps-Universität Marburg, 35032 Marburg, Germany \\ $\ddagger$ Department of Chemistry, University of California, Irvine, 1102 Natural Sciences II, \\ Irvine, CA 92697-2025, USA \\ E-mail: yannick.franzke@chemie.uni-marburg.de; yujm1@uci.edu
}

\begin{abstract}
We present an exact two-component (X2C) ansatz for the EPR g-tensor using gaugeincluding atomic orbitals (GIAOs) and a magnetically balanced basis set expansion. In contrast to previous $\mathrm{X} 2 \mathrm{C}$ and "fully" relativistic ansätze for the g-tensor, this implementation results in a gauge-origin invariant formalism. Furthermore, the derivatives of the relativistic decoupling matrix are considered to form the complete analytical derivative of the $\mathrm{X} 2 \mathrm{C}$ Hamiltonian. To reduce the associated computational costs, we apply the diagonal local approximation to the unitary decoupling transformation (DLU) and the (multipole-accelerated) resolution of the identity approximation. The $\mathrm{X} 2 \mathrm{C}$ ansatz is compared to Douglas-Kroll-Hess theory and the zeroth-order regular approximation for 11 diatomic molecules. The impact of the relativistic Hamiltonian, the basis set, and the density functional approximation is subsequently assessed for
\end{abstract}


a set of 17 transition-metal complexes to complement our previous work on the hyperfine coupling constant [DOI: 10.33774/chemrxiv-2021-wnz1v-v2]. In total, 24 basis sets and 22 density functional approximations are considered. The quasi-relativistic $\mathrm{X} 2 \mathrm{C}$ and DLU-X2C Hamiltonians accurately reproduce the results of the parent "fully" relativistic four-component theory when accounting for two-electron picture-change effects with the modified screened nuclear spin-orbit approximation in the respective one-electron integrals and integral derivatives. Generally, the uncontracted Dyall and segmented-contracted Karlsruhe x2c-type basis sets perform well when compared to large even-tempered basis sets. Moreover, (range-separated) hybrid density functional approximations are needed to match the experimental findings. Here, hybrids based on the meta-generalized gradient approximation are not an a priori improvement. Compared to the other computational parameters, the impact of the GIAOs and the magnetic balance on the actual results in standard calculations is less pronounced. Routine calculations of large molecules are possible with widely available and comparably lowcost hardware as demonstrated for $\left[\mathrm{Pt}\left(\mathrm{C}_{6} \mathrm{Cl}_{5}\right)_{4}\right]^{-}$with 3360 basis functions and three spin-(1/2) $\mathrm{La}(\mathrm{II})$ and $\mathrm{Lu}(\mathrm{II})$ compounds. Both approaches based on a common gauge origin and GIAOs using triple- $\zeta$ basis sets lead to a good agreement with the experimental findings. The best agreement is found with hybrid functionals such as PBE0 and $\omega \mathrm{B} 97 \mathrm{X}-\mathrm{D}$

\section{Introduction}

Electron paramagnetic resonance (EPR) spectroscopy is an essential experimental tool for the study of open-shell systems, especially for transition-metal and lanthanide compounds. The EPR spectrum consists of two important parameters-namely, the g-tensor used to indicate the shift relative to the free electron g-factor and the hyperfine coupling (HFC) constant. An understanding of the spectrum often requires quantum chemical studies to interpret the experimental findings. ${ }^{[-17}$ For example, predictions of EPR parameters from 
theory have helped to investigate the electronic origin of hyperfine clock transitions,, 8 as well as validate trends in magnetic anisotropy orientations,, 9 for distinct classes of lanthanide single molecule magnets (SMMs), to name only a few recent applications. These parameters can be compared directly to those extracted from experimental spectra, which is carried out through fitting the data to an effective spin Hamiltonian. 1

From a theoretical point of view, the g-tensor is an intrinsic relativistic property, and spin-orbit coupling is of the utmost importance for accurate calculations. Keystone methods used in relativistic quantum chemistry 10 include the application of the zeroth-order regular approximation $^{20+22}$ (ZORA) to the g-tensor, $23-26$ the two-component (2c) Douglas-Kroll-Hess (DKH) ansatz by Malkin et al.,27 and the "fully" relativistic four-component (4c) DiracKohn-Sham theory by Komorovsky and co-workers. 28 -31 The latter is undoubtedly the most accurate relativistic treatment. However, it also leads to considerable computational costs compared to the quasi-relativistic two-component ansätze. Today, both ZORA and DKH, as well as other approximate quasi-relativistic Hamiltonians, are replaced by (one-electron) exact two-component $(\mathrm{X} 2 \mathrm{C})$ theory $\stackrel{32+38}{38}$ in many quantum chemistry program suites. $.39-48$ The application of analytical derivative theory 4950 to $\mathrm{X} 2 \mathrm{C}$ is rather straightforward compared to the more involved formulation in $\mathrm{DKH} . \underline{5152}$ Complete analytical derivatives of the X2C Hamiltonian are available for geometry derivatives,,$\frac{53+59}{50}$ electric properties,, $60+63$ and various magnetic properties. $\frac{64}{64}$

The application of X2C to EPR parameters was pioneered in the groups of Autschbach $\frac{75 / 76}{76}$ and Kaupp. ${ }^{[7]}$ These works neglect the derivatives of the relativistic decoupling transformation, which may be motivated by reduced computational costs and the minor impact on the HFC constant. ${ }^{[2 \mid 73}$ Yet, their importance is still to be determined explicitly for the g-tensor. Moreover, the restricted kinetic balance $(\mathrm{RKB})$ condition $\frac{78}{78}$ and a common gauge origin (CGO) are employed for the g-tensor in Ref. 77. From a formal point of view, the RKB condition needs to be generalized to the restricted magnetic balance (RMB) condition, ${ }^{79}$ i.e. the vector potential needs to be included in the basis set expansion of the small compo- 
nent. This ensures the exact non-relativistic limit of the Dirac equation in the presence of an external magnetic field and the errors in the orbital energies are on the order of $\mathcal{O}\left(c^{-4}\right)$ with finite basis sets. ${ }^{[79}$ The use of RMB leads to problematic integrals for the HFC constant and the related nuclear magnetic resonance (NMR) coupling constants in X2C. $\frac{69}{}$ Moreover, the impact was found to be of minor importance for derivatives with respect to the nuclear magnetic moments. $\frac{69171}{}$ However, the integrals can be evaluated straightforwardly for magnetic field derivatives. ${ }^{66}-68 \mid 70$ Thus, the RMB can be used for EPR g-tensors similar to NMR shielding constants ${ }^{66}-68170$ and magnetic circular dichroism. .80

As mentioned previously, a common gauge origin is used in both $\mathrm{X} 2 \mathrm{C}^{77}$ and $4 \mathrm{c}^{28-31}$ calculations of the g-tensor so far. Formally, the g-tensor depends on the chosen gauge origin in finite basis set calculations. $\frac{81}{}$ Consequently, the so-called gauge-including atomic orbitals $^{82183}$ (GIAOs) are employed to remove the gauge-origin dependence. This issue is of great importance for NMR shieldings and GIAOs are thus routinely included in their relativistic calculation. $\underline{66-68 / 70 \mid 84}$ In contrast to NMR shieldings, the g-tensor is a global property of the full molecule and the gauge dependence is less pronounced. $\frac{92 \mid 93}{}$ However, GIAOs are still commonly employed in non-relativistic calculations ${ }^{93}$ and their use in relativistic calculations is also desirable. For instance, a two-component gauge-origin invariant ZORA ansatz was implemented by Verma and Autschbach. 26

Therefore, we extend the available X2C framework for EPR g-tensors in four aspects. First, the derivatives of the unitary decoupling transformation are included to arrive at the complete derivative of the $\mathrm{X} 2 \mathrm{C}$ Hamiltonian. Second, the magnetic balance condition ${ }^{[79}$ is employed. Third, gauge-including atomic orbitals ${ }^{82 / 83}$ are used for the basis set expansion. Fourth, the diagonal local approximation to the unitary decoupling transformation $\frac{99}{}$ (DLU) is introduced for g-tensors, as solving the one-electron $\mathrm{X} 2 \mathrm{C}$ response equations for the decoupling derivatives becomes demanding for large molecules, and local approximations are therefore desirable $\mathrm{e}^{100}-103$ to perform routine calculations of large molecules and metal clusters. 104,107 


\section{Theory}

\subsection{Notation}

We use the same notation as in our work on the HFC constant. ${ }^{73} \mathrm{~L}$ and $\mathrm{S}$ refer to the large and small component, respectively. The subscripts + and - indicate the states of the negative- and positive-energy subspace, which are commonly called positronic and electronic states. $\mathbb{M}$ denotes a matrix in the $4 c$ space, while matrices in the space of spin-free onecomponent (1c) basis functions $\left\{\lambda_{\mu}\right\}$ are indicated as $\mathbf{M}$ and matrices in the 2c space $\left(\left\{\phi_{\mu}\right\}\right.$ $\left.=\left\{\lambda_{\mu}\right\} \otimes\{\alpha, \beta\}\right)$ are indicated as $\boldsymbol{M}$. Cartesian coordinates are denoted with the subscripts $u, v$ while the superscript $B$ indicates derivatives that are formed according to

$$
\boldsymbol{h}_{u}^{B}=\left(\frac{\mathrm{d} \boldsymbol{h}}{\mathrm{d} B_{u}}\right)_{B_{u}=0}
$$

We use Gaussian-based atomic units throughout this work.

\subsection{Definition of the EPR g-Tensor}

We use the approach of Malkin et al. for the relativistic g-tensor ${ }^{27 \mid 28}$

$$
g_{u v}=\left.\frac{\mathrm{d}^{2} E}{\mathrm{~d} B_{u} \mathrm{~d} S_{v}}\right|_{\vec{B}=0}=\left.\frac{2 c}{\left\langle\tilde{S}_{v}\right\rangle} \frac{\mathrm{d} E\left(J_{v}, \vec{B}\right)}{\mathrm{d} B_{u}}\right|_{\vec{B}=0}
$$

with the external magnetic field $\vec{B}$ and the effective spin $\tilde{\vec{S}}$. The energy is calculated with the non-collinear approach, $\frac{108}{10}$ where the magnetization vector $\vec{J}$ and the spin $\vec{S}$ are aligned along three orthogonal axes $v$. The effective spin is determined by the configuration of the electronic ground state, and converged orbitals of a 1c calculation are needed to ensure a good starting point. We note in passing that Verma and Autschbach used a generalized collinear ansatz with a chosen spin quantization for the EPR g-tensor ${ }^{26}$ and the HFC tensor $\frac{109}{1 n}$ a ZORA framework. 


\subsection{X2C Hamiltonian in the Presence of a Magnetic Field}

Magnetic perturbations are introduced into the Dirac equation with the principle of minimal coupling or minimal substitution. $\frac{110}{10}$ The linear momentum operator $\hat{\vec{p}}$ is generalized to $\hat{\vec{\pi}}$ according to

$$
\hat{\vec{p}} \longrightarrow \hat{\vec{\pi}}=\hat{\vec{p}}+\frac{1}{c} \hat{\overrightarrow{\mathcal{A}}}
$$

where $\hat{\overrightarrow{\mathcal{A}}}$ denotes the vector potential. For g-tensors, the vector potential of a static and homogeneous external magnetic field reads

$$
\begin{aligned}
\overrightarrow{\mathcal{A}}_{O}(\vec{r}) & =\frac{1}{2} \vec{B} \times \vec{r}_{O} \\
\vec{r}_{O} & =\vec{r}-\vec{R}_{O}
\end{aligned}
$$

$\vec{R}_{O}$ refers to the gauge origin, which bears no physical meaning and the obtained molecular properties should be gauge-origin independent. Therefore, explicit field-dependent basis functions, the so-called gauge-including atomic orbitals or London orbitals, are introduced $^{[2283}$

$$
\begin{aligned}
\lambda_{\mu}(\vec{B}, \vec{r}) & =\exp \left(-\mathrm{i} \Lambda_{\mu O}\right) \lambda_{\mu}(\vec{r}) \\
\Lambda_{\mu O}(\vec{B}, \vec{r}) & =\frac{1}{2 c}\left(\vec{R}_{\mu O} \times \vec{r}\right) \cdot \vec{B}
\end{aligned}
$$

Here, a (real) field-independent basis function $\lambda_{\mu}$ is augmented by a complex phase prefactor. We use the notation $\vec{R}_{\mu O}=\vec{R}_{\mu}-\vec{R}_{O}$, where $\vec{R}_{\mu}$ denotes the atom center of the respective basis function. Note that we use the Coulomb gauge in this work.

The one-electron Dirac-Hamilton operator in the presence of an external magnetic field reads

$$
\hat{h}_{\mathrm{D}}=c \vec{\alpha} \cdot \hat{\vec{\pi}}+\left(\beta-I_{4}\right) c^{2}+\hat{V}
$$


Here, $\alpha$ and $\beta$ are trace-less quantities defined as

$$
\alpha_{i}=\left(\begin{array}{cc}
0_{2} & \sigma_{i} \\
\sigma_{i} & 0_{2}
\end{array}\right), \quad \beta=\left(\begin{array}{cc}
\sigma_{0} & 0_{2} \\
0_{2} & -\sigma_{0}
\end{array}\right)
$$

$\vec{\sigma}=\left(\sigma_{x}, \sigma_{y}, \sigma_{z}\right)$, is a vector consisting of the Pauli spin matrices

$$
\sigma_{x}=\left(\begin{array}{cc}
0 & 1 \\
1 & 0
\end{array}\right), \quad \sigma_{y}=\left(\begin{array}{cc}
0 & -\mathrm{i} \\
\mathrm{i} & 0
\end{array}\right), \quad \sigma_{z}=\left(\begin{array}{cc}
1 & 0 \\
0 & -1
\end{array}\right)
$$

while $\sigma_{0}$ denotes the $(2 \times 2)$ identity matrix. $\hat{V}$ is the electron-nucleus potential and $I_{4}$ is the $(4 \times 4)$ unit matrix. The basis set expansion using the restricted magnetic balance condition reads $^{79}$

$$
\begin{aligned}
\left|\psi_{i}^{L}\right\rangle & =\sum_{\mu} C_{\mu i}^{L} e^{-\mathrm{i} \Lambda_{\mu O}}\left|\phi_{\mu}\right\rangle \\
\left|\psi_{i}^{S}\right\rangle & =\sum_{\mu} C_{\mu i}^{S} e^{-\mathrm{i} \Lambda_{\mu O} O} \frac{1}{2 c} \vec{\sigma} \cdot\left(\hat{\vec{p}}+\frac{1}{c} \hat{\overrightarrow{\mathcal{A}}}\right)\left|\phi_{\mu}\right\rangle
\end{aligned}
$$

where we used the identities of Ref. 79 to move the GIAO prefactor to the left of the generalized momentum in Eq. 12, This basis set expansion results in the Dirac equation in a matrix representation according to $66-68 \mid 70$

$$
\mathbb{D} \mathbb{C}=\mathbb{M} \mathbb{C E}
$$

In the super-matrix form, this reads

$$
\left(\begin{array}{cc}
\boldsymbol{V} & \boldsymbol{\Pi}^{\dagger} \\
\boldsymbol{\Pi} & \left(\frac{1}{4 c^{2}} \boldsymbol{W}-\boldsymbol{T}\right)
\end{array}\right)\left(\begin{array}{cc}
\boldsymbol{C}_{-}^{\mathrm{L}} & \boldsymbol{C}_{+}^{\mathrm{L}} \\
\boldsymbol{C}_{-}^{\mathrm{S}} & \boldsymbol{C}_{+}^{\mathrm{S}}
\end{array}\right)=\left(\begin{array}{cc}
\boldsymbol{S} & \mathbf{0}_{2} \\
\mathbf{0}_{2} & \frac{1}{2 c^{2}} \boldsymbol{T}
\end{array}\right)\left(\begin{array}{cc}
\boldsymbol{C}_{-}^{\mathrm{L}} & \boldsymbol{C}_{+}^{\mathrm{L}} \\
\boldsymbol{C}_{-}^{\mathrm{S}} & \boldsymbol{C}_{+}^{\mathrm{S}}
\end{array}\right)\left(\begin{array}{ll}
\boldsymbol{\epsilon}_{-} & \mathbf{0}_{2} \\
\boldsymbol{0}_{2} & \boldsymbol{\epsilon}_{+}
\end{array}\right)
$$

The overlap matrix $\boldsymbol{S}$ and the potential matrix $\boldsymbol{V}$ are well known from non-relativistic 
quantum chemistry. These matrices are block-diagonal in the $(2 \times 2)$ superspace and are given by

$$
\begin{aligned}
& \boldsymbol{S}=\left(\begin{array}{ll}
\mathbf{S} & 0 \\
0 & \mathbf{S}
\end{array}\right), \mathbf{S}_{\mu \nu}=\left\langle\lambda_{\mu}\left|e^{i \Lambda_{\mu \nu}}\right| \lambda_{\nu}\right\rangle \\
& \boldsymbol{V}=\left(\begin{array}{cc}
\mathbf{V} & 0 \\
0 & \mathbf{V}
\end{array}\right), \mathbf{V}_{\mu \nu}=\left\langle\lambda_{\mu}\left|e^{i \Lambda_{\mu \nu}} \hat{V}\right| \lambda_{\nu}\right\rangle
\end{aligned}
$$

All other matrices are not block-diagonal anymore and read

$$
\begin{aligned}
\boldsymbol{\Pi}_{\mu \nu}^{\dagger} & =\frac{1}{2}\left\langle\phi_{\mu}\left|e^{i \Lambda_{\mu \nu}}\left[\vec{\sigma} \cdot\left(\hat{\vec{p}}+\frac{1}{c} \hat{\overrightarrow{\mathcal{A}}}\right)\right]\left[\vec{\sigma} \cdot\left(\hat{\vec{p}}+\frac{1}{c} \hat{\overrightarrow{\mathcal{A}}}_{\nu}\right)\right]\right| \phi_{\nu}\right\rangle \\
\boldsymbol{\Pi}_{\mu \nu} & =\frac{1}{2}\left\langle\phi_{\mu}\left|e^{i \Lambda_{\mu \nu}}\left[\vec{\sigma} \cdot\left(\hat{\vec{p}}+\frac{1}{c} \hat{\overrightarrow{\mathcal{A}}}_{\nu}\right)\right]\left[\vec{\sigma} \cdot\left(\hat{\vec{p}}+\frac{1}{c} \hat{\overrightarrow{\mathcal{A}}}_{\nu}\right)\right]\right| \phi_{\nu}\right\rangle \\
\boldsymbol{W}_{\mu \nu} & =\left\langle\phi_{\mu}\left|e^{i \Lambda_{\mu \nu}}\left[\vec{\sigma} \cdot\left(\hat{\vec{p}}+\frac{1}{c} \hat{\overrightarrow{\mathcal{A}_{\nu}}}\right)\right] \hat{V}\left[\vec{\sigma} \cdot\left(\hat{\vec{p}}+\frac{1}{c} \hat{\overrightarrow{\mathcal{A}_{\nu}}}\right)\right]\right| \phi_{\nu}\right\rangle \\
\boldsymbol{T}_{\mu \nu} & =\frac{1}{2}\left\langle\phi_{\mu}\left|e^{i \Lambda_{\mu \nu}}\left[\vec{\sigma} \cdot\left(\hat{\vec{p}}+\frac{1}{c} \hat{\overrightarrow{\mathcal{A}}}_{\nu}\right)\right]\left[\vec{\sigma} \cdot\left(\hat{\vec{p}}+\frac{1}{c} \hat{\overrightarrow{\mathcal{A}}}_{\nu}\right)\right]\right| \phi_{\nu}\right\rangle
\end{aligned}
$$

Here, the generalized momentum matrix $\boldsymbol{\Pi}$ and the small-small block of the metric $\boldsymbol{T}$ are identical due to the magnetically balanced basis set expansion. In a restricted kinetic balanced basis, ${ }^{[78}$ these matrices differ as $\boldsymbol{T}$ becomes independent of the vector potential. Note that the structure of these matrices is similar to those of NMR shielding constants. $\frac{[66-68 \mid 70}{6}$

The four-component Dirac Hamiltonian can be reduced to a two-component form with a unitary transformation as suggested by Foldy and Wouthuysen 111

$$
\mathbb{U}^{\dagger} \mathbb{D} \mathbb{U}=\left(\begin{array}{cc}
\boldsymbol{h}^{+} & \mathbf{0}_{2} \\
\mathbf{0}_{2} & \boldsymbol{h}^{-}
\end{array}\right) \quad \text { with } \quad \mathbb{U}^{\dagger} \mathbb{U}=\mathbb{U}^{\dagger}=\mathbb{I}_{4}
$$

Heully et al. later derived the explicit expression of the unitary matrix. ${ }^{112}$ In X2C, this unitary transformation is carried out for the matrix representation of the Dirac Hamiltonian in one step. Therefore, the X2C Hamiltonian $\frac{32-38}{38}$ in the presence of an external magnetic 
field is then given by

$$
\boldsymbol{h}^{+}=\boldsymbol{R}^{\dagger} \boldsymbol{L} \boldsymbol{R}
$$

with the matrix representation of the normalized elimination of the small component (NESC) operator $[131116$

$$
\boldsymbol{L}=\boldsymbol{V}+\boldsymbol{X}^{\dagger} \boldsymbol{\Pi}+\boldsymbol{\Pi}^{\dagger} \boldsymbol{X}+\boldsymbol{X}^{\dagger}\left(\frac{1}{4 c^{2}} \boldsymbol{W}-\boldsymbol{T}\right) \boldsymbol{X}
$$

Note that all one-electron integrals explicitly depend on the external magnetic field due to the use of GIAOs. Moreover, $\boldsymbol{\Pi}, \boldsymbol{T}$, and $\boldsymbol{W}$ include a dependence on the magnetic field due to the restricted magnetic balance condition. The generalized momentum matrix $\boldsymbol{\Pi}$ is the only matrix, where the dependence also arises due to the generalized momentum operator $\hat{\vec{\pi}}$ and its vector potential $\hat{\overrightarrow{\mathcal{A}}}$. In X2C, the decoupling matrix $\boldsymbol{X}$ is computed based on a diagonalization of the Dirac matrix $\mathbb{D}$. The decoupling matrix reads

$$
\boldsymbol{X}=\boldsymbol{C}_{+}^{\mathrm{S}}\left(\boldsymbol{C}_{+}^{\mathrm{L}}\right)^{-1}
$$

Additionally, the relativistic metric $\mathbb{M}$ needs to be transformed to the non-relativistic metric $\boldsymbol{S}$. This introduces the renormalization matrix $\boldsymbol{R}$, which allows one to integrate the resulting $\mathrm{X} 2 \mathrm{C}$ Hamiltonian into the existing non-relativistic $1 \mathrm{c}$ or $2 \mathrm{c}$ infrastructure. The renormalization matrix is calculated as ${ }^{37}$

$$
\begin{aligned}
\boldsymbol{R} & =\boldsymbol{S}^{-1 / 2}\left(\boldsymbol{S}^{-1 / 2} \tilde{\boldsymbol{S}} \boldsymbol{S}^{-1 / 2}\right)^{-1 / 2} \boldsymbol{S}^{1 / 2} \\
\tilde{\boldsymbol{S}} & =\boldsymbol{S}+\frac{1}{2 c^{2}} \boldsymbol{X}^{\dagger} \boldsymbol{T} \boldsymbol{X}
\end{aligned}
$$

using the metric of the large component $\tilde{\boldsymbol{S}}$, for which the relation

$$
C_{L+}^{\dagger} \tilde{S} C_{L+}=I
$$

holds. This one-electron Hamiltonian is then commonly combined with the non-relativistic 
two-electron interaction. Herein, we account for the missing two-electron picture-change correction with the modified screened nuclear spin-orbit (mSNSO) ansatz, $\underline{57|61| 117 / 118}$ as this significantly improves the results for EPR parameters. $\stackrel{73777}{77}$

\subsection{Gauge-Invariant Formalism for EPR g-Tensors}

The energy derivatives are now formed in the same manner as done in non-relativistic quantum chemistry. Thus, no derivatives of the density matrix are required.1191120 Carrying out the energy derivative for the g-tensor in Eq. 2 consqeuently leads to working equations similar to two-component geometry gradients $\underline{57 / 58 / 121}$

$$
\frac{\mathrm{d} E}{\mathrm{~d} B_{u}}=\operatorname{tr}\left[\boldsymbol{P}\left(\vec{J}_{v}\right) \boldsymbol{h}_{u}^{B}\right]+\frac{1}{2} \operatorname{tr}\left[\boldsymbol{G}\left(\vec{J}_{v}\right) \boldsymbol{\Gamma}_{u}^{B}\left(\vec{J}_{v}\right)\right]+E_{\mathrm{XC}, u}^{B}\left(\overrightarrow{J_{v}}\right)-\operatorname{tr}\left[\boldsymbol{Z}\left(\vec{J}_{v}\right) \boldsymbol{S}_{u}^{B}\right]
$$

Here, $\boldsymbol{P}$ and $\boldsymbol{G}$ are the one and two-electron density matrix, while $\boldsymbol{Z}$ is the energy-weighted

density matrix. $\frac{119 \mid 120}{h}$ and $\boldsymbol{\Gamma}$ are the one-electron Hamiltonian and the two-electron integrals, respectively. $E_{\mathrm{XC}}$ is the exchange-correlation energy in density functional theory (DFT). The one-electron density matrix $\boldsymbol{P}$ reads

$$
\boldsymbol{P}=\left(\begin{array}{ll}
\mathbf{P}^{\alpha \alpha} & \mathbf{P}^{\alpha \beta} \\
\mathbf{P}^{\beta \alpha} & \mathbf{P}^{\beta \beta}
\end{array}\right)
$$

with the one-component matrices

$$
\begin{aligned}
\operatorname{Re}\left(P_{\mu \nu}^{\sigma_{1} \sigma_{2}}\right) & =\sum_{i} n_{i}\left[\operatorname{Re}\left(c_{\mu i}^{\sigma_{1}}\right) \operatorname{Re}\left(c_{\nu i}^{\sigma_{2}}\right)+\operatorname{Im}\left(c_{\mu i}^{\sigma_{1}}\right) \operatorname{Im}\left(c_{\nu i}^{\sigma_{2}}\right)\right] \\
\operatorname{Im}\left(P_{\mu \nu}^{\sigma_{1} \sigma_{2}}\right) & =\sum_{i} n_{i}\left[-\operatorname{Re}\left(c_{\mu i}^{\sigma_{1}}\right) \operatorname{Im}\left(c_{\nu i}^{\sigma_{2}}\right)+\operatorname{Im}\left(c_{\mu i}^{\sigma_{1}}\right) \operatorname{Re}\left(c_{\nu i}^{\sigma_{2}}\right)\right]
\end{aligned}
$$

$n_{i}$ is the occupation number of the corresponding two-component spinor and the superscript $\sigma$ denotes the Kramers index $(\sigma=\alpha, \beta) . c_{\mu i}$ are the spinor coefficients from a self-consistent field $(\mathrm{SCF})$ procedure. The two-electron density matrix is formed similarly. $\frac{121}{1}$ Note that 
these matrices are constructed from the spinors with $\vec{J}$ aligned along the $v$ axis. As $\boldsymbol{S}_{u}^{B}$ is an antisymmetric and purely imaginary matrix, which is diagonal in the $(2 \times 2)$ superspace, we only need the respective $\alpha \alpha$ and $\beta \beta$ blocks of the energy-weighted density matrix, given by the spinor coefficients and energies, according to

$$
\operatorname{Im}\left[\mathbf{Z}_{\mu \nu}^{\sigma \sigma}\right]=\sum_{i} n_{i}\left[-\operatorname{Re}\left(c_{\mu i}^{\sigma}\right) \epsilon_{i} \operatorname{Im}\left(c_{\nu i}^{\sigma}\right)+\operatorname{Im}\left(c_{\mu i}^{\sigma}\right) \epsilon_{i} \operatorname{Re}\left(c_{\nu i}^{\sigma}\right)\right]
$$

$n_{i}$ is the occupation number. Therefore, the last term in Eq. 28 is simplified to

$$
\operatorname{tr}\left(\boldsymbol{Z} \boldsymbol{S}_{u}^{B}\right)=-\operatorname{tr}\left(\mathrm{i}\left\{\operatorname{Im}\left[\mathbf{Z}^{\alpha \alpha}\right]+\operatorname{Im}\left[\mathbf{Z}^{\beta \beta}\right]\right\} \mathbf{S}_{u}^{B}\right)
$$

Note that the imaginary part of the energy-weighted density matrix is not needed for relativistic geometry gradients. However, it also arises for geometry gradients in finite magnetic fields. ${ }^{122}$ An important point is that all terms in Eq. 28 except for the one with $\boldsymbol{h}_{u}^{B}$ arise solely due to the basis set expansion with GIAOs. Note that the computation of the two-electron integral derivatives is described in Refs. 123-125 and their generalization to a two-component framework is carried out as described in, e.g., Refs. 121 and 126. Further note that the derivatives of the two-electron integrals in Eq. 28 are known from the right-hand side of the coupled-perturbed Hartree-Fock (CPHF) or Kohn-Sham (CPKS) equations for twocomponent NMR shielding tensors. $\frac{68|88| 90|91| 127}{6}$ The crucial part for a relativistic treatment is the derivative of the one-electron Hamiltonian, which is also very similar to the perturbed density contribution or paramagnetic contribution to the NMR shielding tensor. $\underline{66-68|70| 128}$

Generally, the X2C Hamiltonian derivatives are given by

$$
\boldsymbol{h}_{u}^{+, B}=\boldsymbol{R}_{u}^{\dagger, B} \boldsymbol{L} \boldsymbol{R}+\boldsymbol{R}^{\dagger} \boldsymbol{L}_{u}^{B} \boldsymbol{R}+\boldsymbol{R}^{\dagger} \boldsymbol{L} \boldsymbol{R}_{u}^{B}
$$


where the derivative of the NESC matrix reads

$$
\begin{aligned}
\boldsymbol{L}_{u}^{B}= & \boldsymbol{V}_{u}^{B}+\boldsymbol{\Pi}_{u}^{\dagger, B} \boldsymbol{X}+\boldsymbol{\Pi}^{\dagger} \boldsymbol{X}_{u}^{B}+\boldsymbol{X}_{u}^{\dagger, B} \boldsymbol{\Pi}+\boldsymbol{X}^{\dagger} \boldsymbol{\Pi}_{u}^{B} \\
& +\boldsymbol{X}_{u}^{\dagger, B}\left(\frac{1}{4 c^{2}} \boldsymbol{W}-\boldsymbol{T}\right) \mathbf{X}+\boldsymbol{X}^{\dagger}\left(\frac{1}{4 c^{2}} \boldsymbol{W}_{u}^{B}-\boldsymbol{T}_{u}^{B}\right) \boldsymbol{X}+\boldsymbol{X}^{\dagger}\left(\frac{1}{4 c^{2}} \boldsymbol{W}-\boldsymbol{T}\right) \boldsymbol{X}_{u}^{B}
\end{aligned}
$$

Thus, the one-electron integral derivatives of all matrices involved in the NESC matrix are needed. Additionally, the derivative of the overlap matrix is required for $\boldsymbol{R}_{u}^{B}$ (vide infra) The derivatives of the potential and the overlap are

$$
\begin{aligned}
&\left(\frac{\partial \mathbf{V}_{\mu \nu}}{B_{u}}\right)_{0}=\frac{\mathrm{i}}{2 c}\left\langle\lambda_{\mu}\left|\left(\vec{R}_{\mu \nu} \times \hat{\vec{r}}\right)_{u} \hat{V}\right| \lambda_{\nu}\right\rangle \\
&\left(\frac{\partial \mathbf{S}_{\mu \nu}}{B_{u}}\right)_{0}=\frac{\mathrm{i}}{2 c}\left\langle\lambda_{\mu}\left|\left(\vec{R}_{\mu \nu} \times \hat{\vec{r}}\right)_{u}\right| \lambda_{\nu}\right\rangle
\end{aligned}
$$

These derivatives are purely imaginary and antisymmetric. Moreover, they are identical to the one-component non-relativistic integral derivatives. The other derivatives are not block-diagonal in the $2 \mathrm{c}$ space and read $\underline{66}-68$

$$
\begin{aligned}
& \left(\frac{\partial \boldsymbol{T}_{\mu \nu}}{\partial B_{u}}\right)_{0}=\frac{\mathrm{i}}{4 c} \sigma_{0}\left\langle\lambda_{\mu}\left|\left(\vec{R}_{\mu \nu} \times \hat{\vec{r}}\right)_{u} \hat{\vec{p}}^{2}\right| \lambda_{\nu}\right\rangle+\frac{1}{2 c} \sigma_{0}\left\langle\lambda_{\mu}\left|\left(\hat{\vec{r}}_{\nu} \times \hat{\vec{p}}\right)_{u}\right| \lambda_{\nu}\right\rangle+\frac{1}{2 c} \sigma_{u}\left\langle\lambda_{\mu} \mid \lambda_{\nu}\right\rangle \\
& \left(\frac{\partial \boldsymbol{\Pi}_{\mu \nu}^{\dagger}}{\partial B_{u}}\right)_{0}=\frac{\mathrm{i}}{4 c} \sigma_{0}\left\langle\lambda_{\mu}\left|\left(\vec{R}_{\mu \nu} \times \hat{\vec{r}}\right)_{u} \hat{\vec{p}}^{2}\right| \lambda_{\nu}\right\rangle+\frac{1}{2 c} \sigma_{0}\left\langle\lambda_{\mu}\left|\left(\hat{\vec{r}}_{\nu} \times \hat{\vec{p}}\right)_{u}\right| \lambda_{\nu}\right\rangle+\frac{1}{2 c} \sigma_{u}\left\langle\lambda_{\mu} \mid \lambda_{\nu}\right\rangle \\
& \left(\frac{\partial \boldsymbol{W}_{\mu \nu}}{\partial B_{u}}\right)_{0}=\frac{\mathrm{i}}{2 c} \sigma_{0}\left\langle\lambda_{\mu}\left|\left(\vec{R}_{\mu \nu} \times \hat{\vec{r}}\right)_{u} \hat{\vec{p}} \cdot \hat{V} \hat{\vec{p}}\right| \lambda_{\nu}\right\rangle+\frac{1}{2 c} \sigma_{0}\left\langle\lambda_{\mu}\left|\left(\hat{\vec{r}}{ }_{\nu} \times \hat{\vec{p}}\right)_{u} \hat{V}+\hat{V}(\hat{\vec{r}} \times \hat{\vec{p}})_{u}\right| \lambda_{\nu}\right\rangle \\
& +\frac{1}{2 c}\left\langle\lambda_{\mu}\left|\left(\vec{R}_{\mu \nu} \times \hat{\vec{r}}\right)_{u} \mathrm{i} \vec{\sigma} \cdot(\hat{\vec{p}} \times \hat{V} \hat{\vec{p}})\right| \lambda_{\nu}\right\rangle+\frac{1}{c} \sigma_{u}\left\langle\lambda_{\mu}|\hat{V}| \lambda_{\nu}\right\rangle \\
& +\left\langle\lambda_{\mu}\left|\frac{\mathrm{i}}{2 c} \sigma_{u}(\hat{\vec{p}} \hat{V}) \cdot \hat{\vec{r}}_{\nu}-\frac{\mathrm{i}}{2 c}(\hat{\vec{p}} \hat{V})_{u}\left(\vec{\sigma} \cdot \hat{\vec{r}}_{\nu}\right)\right| \lambda_{\nu}\right\rangle
\end{aligned}
$$

The expressions for the derivatives of $\boldsymbol{T}$ and $\boldsymbol{\Pi}$ are obtained by making use of the commutator for $\hat{\vec{r}}$ and $\hat{\vec{p}}$. Note that the first-order Dirac matrix is still Hermitian. The spin-free part of all matrices is antisymmetric and purely imaginary. The spin-dependent contributions of the relativistically modified potential $\boldsymbol{W}$ and its derivatives are rescaled in the (modified) 


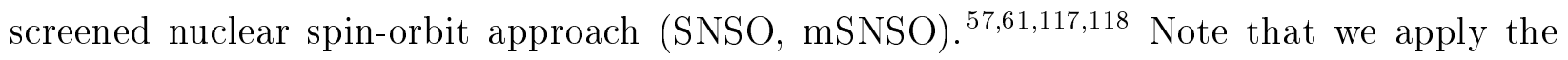
SNSO and mSNSO to the integrals directly. 129

The derivatives of the decoupling matrix $\boldsymbol{X}_{u}^{B}$ are obtained using one-electron response theory as reviewed in the appendix of Ref. 70. We refer to this reference for a detailed derivation and overview of related approaches. The working equations are

$$
\boldsymbol{X}_{u}^{B}=\left(\boldsymbol{C}_{-}^{S}-\boldsymbol{X} \mathbf{C}_{-}^{L}\right)\left(\boldsymbol{U}_{u}^{B}\right)_{-+} \boldsymbol{C}_{+}^{L, \dagger} \tilde{\boldsymbol{S}}
$$

with the orbital rotation matrix

$$
\left(\boldsymbol{U}_{u,-+}^{B}\right)_{k l}=\frac{\left(\tilde{\boldsymbol{D}}_{u,-+}^{B}\right)_{k l}-\left(\tilde{\boldsymbol{M}}_{u,-+}^{B}\right)_{k l}\left(\boldsymbol{E}_{++}\right)_{l l}}{\left(\boldsymbol{E}_{++}\right)_{l l}-\left(\boldsymbol{E}_{--}\right)_{k k}}
$$

The derivatives of the Dirac matrix and the metric of Eq. 14 are transformed to the basis of the unperturbed solutions according to

$$
\begin{aligned}
\tilde{\boldsymbol{D}}_{u,-+}^{B}= & \boldsymbol{C}_{-}^{L, \dagger} \boldsymbol{V}_{u}^{B} \boldsymbol{C}_{+}^{L}+\boldsymbol{C}_{-}^{L, \dagger} \boldsymbol{\Pi}_{u}^{\dagger, B} \boldsymbol{C}_{+}^{S}+\boldsymbol{C}_{-}^{S, \dagger} \boldsymbol{\Pi}_{u}^{B} \boldsymbol{C}_{+}^{L} \\
& +\frac{1}{4 c^{2}} \boldsymbol{C}_{-}^{S, \dagger} \boldsymbol{W}_{u}^{B} \boldsymbol{C}_{+}^{S}-\boldsymbol{C}_{-}^{S, \dagger} \boldsymbol{T}_{u}^{B} \boldsymbol{C}_{+}^{S} \\
\tilde{\boldsymbol{M}}_{u,-+}^{B}= & \boldsymbol{C}_{-}^{L, \dagger} \boldsymbol{S}_{u}^{B} \boldsymbol{C}_{+}^{L}+\frac{1}{2 c^{2}} \boldsymbol{C}_{-}^{S, \dagger} \boldsymbol{T}_{u}^{B} \boldsymbol{C}_{+}^{S}
\end{aligned}
$$

The derivatives of the renormalization matrix are calculated by solving the Sylvester matrix equation

$$
\boldsymbol{R} \boldsymbol{R}_{u}^{B}+\boldsymbol{R}_{u}^{B} \boldsymbol{R}=\tilde{\boldsymbol{S}}^{-1}\left(\mathbf{S}_{u}^{B}-\tilde{\boldsymbol{S}}_{u}^{B} \boldsymbol{R} \boldsymbol{R}\right)
$$

using a generalized eigenvalue decomposition method. ${ }^{56}$ The derivative of $\tilde{\boldsymbol{S}}$ is given by

$$
\tilde{\boldsymbol{S}}_{u}^{B}=\boldsymbol{S}_{u}^{B}+\frac{1}{2 c^{2}} \boldsymbol{X}_{u}^{\dagger, B} \boldsymbol{T} \boldsymbol{X}+\frac{1}{2 c^{2}} \boldsymbol{X}^{\dagger} \boldsymbol{T}_{u}^{B} \boldsymbol{X}+\frac{1}{2 c^{2}} \boldsymbol{X}^{\dagger} \boldsymbol{T} \boldsymbol{X}_{u}^{B}
$$

These Sylvester matrix equations are obtained by differentiating the quadratic expression 
for the renormalization matrix ${ }^{116}$

$$
\boldsymbol{R} \boldsymbol{R}=\tilde{\boldsymbol{S}}^{-1} \boldsymbol{S}
$$

with respect to the magnetic field. The simplification of this formalism with a common gauge origin and the RKB condition is discussed in the appendix A. There, we also outline how our EPR implementation can be used for paramagnetic NMR (pNMR) shielding tensors.

\subsection{Local Approximation to the X2C Hamiltonian}

All X2C steps are carried out in the uncontracted or primitive basis set. Thus, the $\mathcal{O}\left(N^{3}\right)$ scaling of the diagonalization may limit the applicability of $\mathrm{X} 2 \mathrm{C}$ for very large molecular systems. ${ }^{101}$ Here, $N$ denotes the number of basis functions. Therefore, local approximations are commonly introduced. $\frac{100}{}$ Herein, we chose the diagonal local approximation to the unitary decoupling transformation. ${ }^{99}$ In DLU, the unitary decoupling transformation matrix $\mathbb{U}$ is approximated as a direct sum of atomic contributions according to

$$
\begin{aligned}
\boldsymbol{U}^{\mathrm{LL}} & =\bigoplus_{A} \boldsymbol{U}_{A A}^{\mathrm{LL}}=\bigoplus_{A} \boldsymbol{R}_{A A} \\
\boldsymbol{U}^{\mathrm{SL}} & =\bigoplus_{A} \boldsymbol{U}_{A A}^{\mathrm{SL}}=\bigoplus_{A} \boldsymbol{X}_{A A} \boldsymbol{R}_{A A}
\end{aligned}
$$

Note that the (relativistically modified) potential still includes the sum over all nuclei. We denote an atomic diagonal block $A A$ and a general block $A B$, where the atomic blocks are formed according to the atom centers of the basis functions of the respective matrix elements. Therefore, the Hamiltonian is simplified to

$$
\begin{aligned}
\boldsymbol{h}_{A B}^{+}= & \boldsymbol{R}_{A A}^{\dagger}\left(\boldsymbol{V}_{A B}+\boldsymbol{\Pi}_{A B}^{\dagger} \boldsymbol{X}_{B B}+\boldsymbol{X}_{A A}^{\dagger} \boldsymbol{\Pi}_{A B}\right) \boldsymbol{R}_{B B} \\
& +\boldsymbol{R}_{A A}^{\dagger}\left(\boldsymbol{X}_{A A}^{\dagger}\left[\frac{1}{4 c^{2}} \boldsymbol{W}_{A B}-\boldsymbol{T}_{A B}\right] \boldsymbol{X}_{B B}\right) \boldsymbol{R}_{B B}
\end{aligned}
$$

Analytical derivatives are formed with the product rule. Thus, only the atomic diagonal block of $\boldsymbol{X}$ and $\boldsymbol{R}$ as well as their derivatives are required. However, both the atomic 
diagonal and atomic off-diagonal blocks of the integral derivatives are needed according to

$$
\begin{aligned}
\boldsymbol{h}_{A B}^{+, B}= & \boldsymbol{R}_{A A, u}^{\dagger, B} \boldsymbol{L}_{A B} \boldsymbol{R}_{B B}^{\dagger}+\boldsymbol{R}_{A A}^{\dagger} \boldsymbol{L}_{A B} \boldsymbol{R}_{B B, u}^{B} \\
& +\boldsymbol{R}_{A A}^{\dagger}\left(\boldsymbol{V}_{A B, u}^{B}+\boldsymbol{\Pi}_{A B, u}^{\dagger, B} \boldsymbol{X}_{B B}+\boldsymbol{X}_{A A}^{\dagger} \boldsymbol{\Pi}_{A B, u}^{B}\right) \boldsymbol{R}_{B B} \\
& +\boldsymbol{R}_{A A}^{\dagger}\left(\boldsymbol{\Pi}_{A B}^{\dagger} \boldsymbol{X}_{B B, u}^{B}+\boldsymbol{X}_{A A, u}^{\dagger, B} \boldsymbol{\Pi}_{A B}\right) \boldsymbol{R}_{B B} \\
& +\boldsymbol{R}_{A A}^{\dagger}\left(\boldsymbol{X}_{A A, u}^{\dagger, B}\left[\frac{1}{4 c^{2}} \boldsymbol{W}_{A B}-\boldsymbol{T}_{A B}\right] \boldsymbol{X}_{B B}\right) \boldsymbol{R}_{B B} \\
& +\boldsymbol{R}_{A A}^{\dagger}\left(\boldsymbol{X}_{A A}^{\dagger}\left[\frac{1}{4 c^{2}} \boldsymbol{W}_{A B}-\boldsymbol{T}_{A B}\right] \boldsymbol{X}_{B B, u}^{B}\right) \boldsymbol{R}_{B B} \\
& +\boldsymbol{R}_{A A}^{\dagger}\left(\boldsymbol{X}_{A A}^{\dagger}\left[\frac{1}{4 c^{2}} \boldsymbol{W}_{A B, u}^{B}-\boldsymbol{T}_{A B, u}^{B}\right] \boldsymbol{X}_{B B}\right) \boldsymbol{R}_{B B}
\end{aligned}
$$

\section{Implementation}

The calculation of EPR g-tensors with a common gauge origin is implemented into the ridft


SCF iterations. The gauge-invariant ansatz employing GIAOs is implemented into the mpshift module. $\frac{124|125| 136+138}{11}$ The spin-independent integral derivatives using GIAOs are taken from Ref. 70. All new integrals are implemented using a combination of Gauss-Hermite and Gauss-Rys integration ${ }^{[39-142}$ as done previously for other magnetic ${ }^{7173174}$ and electric properties. ${ }^{143}$ The finite nucleus model is available throughout using the finite nucleus exponents of Ref. 144. The (modified) screened-nuclear spin-orbit (SNSO, mSNSO) approximation is available for the spin-dependent integral (derivatives) of the relativistically modified potential $\boldsymbol{W} \cdot \frac{57611117118}{1}$ The response and Sylvester matrix equations are solved by a generalization of the existing one-component routines ${ }^{70}$ as done previously for the HFC constant ${ }^{73}$ and NMR coupling constants. ${ }^{171}$ As our previous works, the implementation is fully integral direct. We use $c=137.0359990840$ a.u ${ }^{145}$ for the speed of light in atomic units. Additional contributions from point charges or the conductor-like screening model ${ }^{[146] 147}$ (COSMO) arise due to the use of GIAOs and are treated without relativistic picture-change correction. Thus, we use the available non-relativistic implementation ${ }^{[125}$ for these terms. 
The ridft module supports the (multipole-accelerated) resolution of the identity approximation (MARI- $J$, RI- $J$, and RI- $K$ ) throughout. .1261148 Additionally, the seminumerical exchange approximation is available. 130131 The mpshift module only supports MARI- $J$ and RI- $J$ for integral derivatives. $\frac{125}{125}$ Note that these do not require changes for a two-component ansatz as the Coulomb term depends on the total density ${ }^{126}$ The analytical exchange integral derivatives with respect to the magnetic fields ${ }^{124}$ are extended to a two-component framework as done previously for energies ${ }^{126}$ and energy gradients. ${ }^{[21]}$ Therefore, the integral routines are able to process the eight density matrices. For the contraction with the density matrix, we construct the symmetric and antisymmetric linear combinations. $\frac{121}{12}$

Density functional approximations are available up to the fourth rung of Jacob's ladder. $\frac{149}{151}$ The kinetic energy density $\tau$ is generalized using the external magnetic field as suggested by Maximoff and Scuseria. ${ }^{\frac{152]}{15}}$ Interfaces to Libxc ${ }^{\frac{153}{155}}$ and XCFun ${ }^{\frac{156}{156}}$ are provided. For this work, the existing one-component implementations ${ }^{125 \mid 136157}$ are extended like done previously for the SCF energy. ${ }^{126}$ Thus, the non-collinear exchange-correlation energy depends on the total density matrix $\mathbf{M}_{0}$ and the three spin vector density matrices $\mathbf{M}_{i}(i=x, y, z)$. These are defined as $\frac{1081158}{10}$

$$
\begin{aligned}
& \mathbf{M}_{0}=\operatorname{Re}\left(\mathbf{P}^{\alpha \alpha}\right)+\operatorname{Re}\left(\mathbf{P}^{\beta \beta}\right) \\
& \mathbf{M}_{x}=\operatorname{Re}\left(\mathbf{P}^{\alpha \beta}\right)+\operatorname{Re}\left(\mathbf{P}^{\beta \alpha}\right) \\
& \mathbf{M}_{y}=\operatorname{Im}\left(\mathbf{P}^{\alpha \beta}\right)-\operatorname{Im}\left(\mathbf{P}^{\beta \alpha}\right) \\
& \mathbf{M}_{z}=\operatorname{Re}\left(\mathbf{P}^{\alpha \alpha}\right)-\operatorname{Re}\left(\mathbf{P}^{\beta \beta}\right)
\end{aligned}
$$

The XC potential follows as

$$
\boldsymbol{V}_{\mathrm{XC}}\left[M_{0}(\vec{r}), M_{i}(\vec{r})\right]=\frac{\delta E_{\mathrm{XC}}\left[M_{0}(\vec{r}), \rho_{i}(\vec{r})\right]}{\delta M_{0}(\vec{r})}+\vec{\sigma}_{i} \frac{\delta E_{\mathrm{XC}}\left[M_{0}(\vec{r}), M_{i}(\vec{r})\right]}{\delta M_{i}(\vec{r})}
$$

and the existing one-component implementation ${ }^{\frac{125|136| 157}{1}}$ can be extended straightforwardly 
based on the existing energy routines. $\frac{126}{12}$

All algebraic operations, transformations, and integral evaluations are parallelized using the OpenMP scheme ${ }^{159 \mid 160}$ and we use the Intel ${ }^{\circledR}$ Math Kernel Library (Intel MKL) throughout this work. Preparation and post-processing scripts are available based on our previous endeavors for the hyperfine coupling matrix. ${ }^{73}$ Three independent calculations based on converged unrestricted Kohn-Sham (UKS) or Hartree-Fock (UHF) orbitals are needed to obtain the complete EPR g-matrix. This matrix is then transformed into its principal axis system, where the g-matrix is diagonal, either through constructing the rank-2 tensor $\left(\boldsymbol{g g}^{\mathrm{T}}\right)$ and subsequently diagonalizing, or by diagonalization of the symmetric part, which is given by $1 / 2\left(\boldsymbol{g}+\boldsymbol{g}^{\mathrm{T}}\right)$. We implemented both ansätze using Python scripts.

\section{Computational Details}

To begin, we compare our newly developed X2C implementation to established two-component DKH and ZORA approaches. A small set of 11 molecules, i.e. RhC, PdH, ZnH, CdH, $\mathrm{HgH}$, $\mathrm{ZnF}, \mathrm{CdF}, \mathrm{HgF}, \mathrm{ZnAg}, \mathrm{CdAg}$, and $\mathrm{HgAg}$, is selected. This set was previously studied with DKH and a common gauge origin in Ref. 27 as well as with ZORA and GIAOs in Ref. 161. For consistency, we use the bond lengths collected in the latter reference. Both the DKH and ZORA calculations are performed with tailored basis sets and the BP86 functional. $162 \mid 163$ Note that the ZORA study is carried out with Slater-type orbitals. Consequently, we also apply basis sets optimized for X2C-namely the x2c-QZVPall-2c basis set ${ }^{164}$ herein. Additionally, we employ the uncontracted Dyall-VTZ basis set for $\mathrm{Rh}, \mathrm{Pd}, \mathrm{Ag}, \mathrm{Cd}$, and $\mathrm{Hg}, 165$ while the decontracted pcS-2 basis is used for H and F. ${ }^{169}$ For Zn, the decontracted pcSseg-2 bases $^{[170}$ are chosen. Results with this basis set combination are listed in the Supporting Information. Additionally, results with the PBE0 $0^{171 \mid 172}$ and $\omega \mathrm{B} 97 \mathrm{X}-\mathrm{D}^{173}$ functionals as well as results with the RKB and RMB condition using a common gauge origin are provided in the Supporting Information. The impact of the structure on the g-tensor is also studied in 
the Supporting Information. Tight SCF thresholds of $10^{-8} \mathrm{E}_{\mathrm{h}}$ and fine grids $\frac{174}{176}$ (grid 4a) are applied. The shift of the principal components of the g-tensor is given relative to the free electron in parts per thousand (ppt), $\Delta \mathrm{g}=\left(g-g_{e}\right) \cdot 1000$.

Furthermore, we perform benchmark calculations to assess the three parameters of a quantum chemical DFT calculation of the g-tensor-namely the Hamiltonian, the basis set, and the density functional approximation (DFA). This complements our previous work on the HFC constant. ${ }^{73}$ First, we consider the 17 transition-metal complexes of Ref. 29, namely $\left[\mathrm{MoNCl}_{4}\right]^{2-},\left[\mathrm{MoOF}_{4}\right]^{-},\left[\mathrm{MoOCl}_{4}\right]^{-},\left[\mathrm{MoOF}_{5}\right]^{2-},\left[\mathrm{MoOBr}_{5}\right]^{2-},\left[\mathrm{WOCl}_{4}\right]^{-},\left[\mathrm{WOF}_{5}\right]^{2-}$, $\left[\mathrm{WOBr}_{5}\right]^{2-},\left[\mathrm{TcNF}_{4}\right]^{-},\left[\mathrm{TcNCl}_{4}\right]^{-},\left[\mathrm{TcNBr}_{4}\right]^{-},\left[\mathrm{ReNF}_{4}\right]^{-},\left[\mathrm{ReNCl}_{4}\right]^{-},\left[\mathrm{ReNBr}_{4}\right]^{-},\left[\mathrm{ReOBr}_{4}\right]$, $\left[\mathrm{ReOF}_{5}\right]^{-}$, and $\left[\mathrm{OsOF}_{5}\right]$, and compare our X2C and DLU-X2C results to available 4c reference data. In line with that reference, we use the RKB condition for the basis set expansion and a common gauge origin placed at the heavy atom center. Decontracted IGLO-III bases are employed for the light elements, namely $\mathrm{N}, \mathrm{F}$, and $\mathrm{Cl}, \stackrel{177}{1}$ while the Dyall-VTZ basis set is used for Br ${ }^{\frac{165[168}{16}}$ and the Dyall-TZ bases for Mo, Tc, W, Re, and Os. ${ }^{166-168}$ The PBE0 $\frac{171 \mid 172}{16}$ functional is chosen with very large grids (grid 5a). $\frac{174-176}{16}$ Furthermore, the mSNSO approximation is applied and an SCF convergence threshold of $10^{-9} \mathrm{E}_{\mathrm{h}}$ is selected. Results without the mSNSO approximation are presented in the Supporting Information. There, we also discuss the impact of diffuse basis functions using the Sapporo-DKH3-QZP-2012-diffuse bases. $\frac{178 \mid 179}{}$ All two-component calculations are performed with converged UKS orbitals. In this work, "PCC" indicates that the derivatives of the decoupling and the renormalization matrices are neglected. Thus, the computational algorithm becomes similar to ordinary picture-change corrections of expectation values. $\frac{143}{2}$ Like Ref. 29 , we use the shift $\Delta \mathrm{g}$ in ppt relative to the g-factor of the free electron. The impact of the balance condition and the gauge-invariant formalism is assessed for the same set of molecules using the described computational settings.

Second, the importance of the basis set is studied. To do so, the basis sets in Tab. 1 are considered. The x2c-SVPall-2c-s and x2c-TZVPall-2c-s bases are obtained by combining 
the extensions for NMR shieldings of Ref. 176 with the 2c extensions of Ref. 132. Note that the comparably flat $p$ and $d$ functions of the NMR extensions were removed to avoid linear dependencies, as these functions were optimized to serve as a minimal version of the $2 \mathrm{c}$ extensions. $\frac{176}{176}$ For convenience, the basis sets are given as part of the Supporting Information. We measure the errors with respect to the large even-tempered basis set of Ref. 164. Note that $\left[\mathrm{TcNCl}_{4}\right]^{-}(4 \mathrm{ppt})$ and $\left[\mathrm{WOF}_{5}\right]^{2-}$ are excluded form the statistical evaluation. Only the DLU-X2C Hamiltonian using the RMB-GIAO approach is discussed in the main text and we refer to the Supporting Information for the results with the CGO ansatz.

Table 1: Considered basis sets and basis set combinations for the light (N, O, F, Cl) and heavy elements (Br, Mo, Tc, W, Re, Os). The suffix "-unc" denotes that the bases are used in the decontracted form. NBF is the total number of spherical AO basis functions. The even-tempered reference basis set ${ }^{176}$ uses 26085 basis functions for the molecular test set (average: 1534). "Avg." denotes the average number of basis functions for a molecule.

\begin{tabular}{|c|c|c|c|c|c|c|}
\hline Basis Set & Light Elements & Ref. & Heavy Elements & Ref. & NBF & Avg. \\
\hline 1 & IGLO-II-unc & 177 & Dyall-VDZ-unc & $165-168$ & 6421 & 378 \\
\hline 2 & IGLO-III-unc & 177 & Dyall-VTZ-unc & $165-168$ & 9176 & 540 \\
\hline 3 & pcS-1-unc & 169 & Dyall-VDZ-unc & $165-168$ & 6742 & 397 \\
\hline 4 & pcS-2-unc & 169 & Dyall-VTZ-unc & $165-168$ & 10140 & 596 \\
\hline 5 & pcS-3-unc & 169 & Dyall-VQZ-unc & $165-168$ & 15217 & 895 \\
\hline 6 & cc-pVDZ-unc & 180181 & Dyall-VDZ-unc & $165-168$ & 6440 & 379 \\
\hline 7 & cc-pVTZ-unc & 180181 & Dyall-VTZ-unc & $165-168$ & 9425 & 554 \\
\hline 8 & cc-pVQZ-unc & $180 \quad 181$ & Dyall-VQZ-unc & $165-168$ & 11634 & 684 \\
\hline 9 & Sapporo-DZP-2012 & 178 & Sapporo-DKH3-DZP-2012 & 178179 & 3369 & 198 \\
\hline 10 & Sapporo-TZP-2012 & 178 & Sapporo-DKH3-TZP-2012 & 178179 & 6213 & 367 \\
\hline 11 & Sapporo-QZP-2012 & 178 & Sapporo-DKH3-QZP-2012 & 178,179 & 9804 & 577 \\
\hline 12 & Jorge-DZP-DKH & 182 & Jorge-DZP-DKH & $182-184$ & 3085 & 181 \\
\hline 13 & Jorge-TZP-DKH & 182 & Jorge-TZP-DKH & $182-184$ & 5038 & 296 \\
\hline 14 & x2c-SVPall-2c & 132 & x2c-SVPall-2c & 132 & 3315 & 195 \\
\hline 15 & x2c-TZVPall-2c & 132 & x2c-TZVPall-2c & 132 & 5095 & 300 \\
\hline 16 & x2c-QZVPall-2c & 164 & x2c-QZVPall-2c & 164 & 8799 & 518 \\
\hline 17 & x2c-SVPall-2c-s & 132176 & x2c-SVPall-2c-s & 132176 & 3671 & 216 \\
\hline 18 & $\mathrm{x} 2 \mathrm{c}-\mathrm{TZVPall}-2 \mathrm{c}-\mathrm{s}$ & 132176 & $\mathrm{x} 2 \mathrm{c}-\mathrm{TZVPall}-2 \mathrm{c}-\mathrm{s}$ & 132176 & 5427 & 319 \\
\hline 16 & $\mathrm{x} 2 \mathrm{c}-\mathrm{QZVPall}-2 \mathrm{c}-\mathrm{s}$ & 164 & x2c-QZVPall-2c-s & 164 & 8946 & 526 \\
\hline 20 & x2c-SVPall-2c-unc & 132 & x2c-SVPall-2c-unc & 132 & 7614 & 448 \\
\hline 21 & x2c-TZVPall-2c-unc & 132 & x2c-TZVPall-2c-unc & 132 & 9104 & 536 \\
\hline 22 & x2c-QZVPall-2c-unc & 164 & x2c-QZVPall-2c-unc & 164 & 14366 & 845 \\
\hline 23 & ANO-R-unc & 185 & ANO-R-unc & 185 & 15764 & 927 \\
\hline 24 & ANO-RCC-unc & 186 & ANO-RCC-unc & 186,187 & 15757 & 927 \\
\hline
\end{tabular}


Third, we study common density functional approximations. As representatives for pure

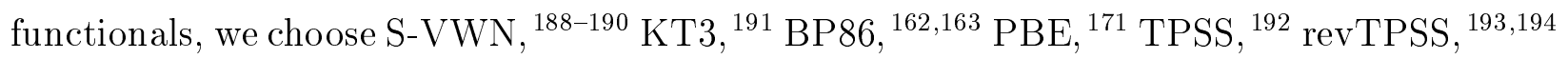
and $r^{2}$ SCAN. ${ }^{195 / 196}$ BH\&HLYP, $163\left[1971198\right.$ B3LYP, ${ }^{1971199}$ PBE0, ${ }^{171172]}$ a modified version of PBE0 including $40 \%$ of Hartree-Fock (HF) exchange (PBE0-40HF), ${ }_{291171172}$ B97, 200 B972, 201 TPSSh, ${ }^{202}$ revTPSSh, $\frac{193194}{2}$ and TPSS0 ${ }^{2021203}$ are selected to cover frequently used global hybrid functionals. For common range-separated hybrids, we employ the CAM-


$\mathrm{D}^{1173}$ functionals. Libxc ${ }^{153}-155$ is used for the range-separated hybrid functionals, KT3, revTPSS(h), TPSS0, $\mathrm{r}^{2} \mathrm{SCAN}$, and B97 throughout this work. We use XCFun ${ }^{156}$ for PBE040HF. Additionally, Hartree-Fock calculations are performed. COSMO is employed with the default parameters to compensate the negative charge ${ }^{146 \mid 147}$ and the x2c-QZVPall-2c basis set is chosen for consistency with our previous studies on the HFC constant. ${ }^{[73}$ Note that COSMO introduces additional integral derivatives due to the GIAOs. Errors are measured with respect to the experimental findings ${ }^{211}[222]$ collected in Ref. 29 ,

Fourth, we apply the mSNSO-DLU-X2C Hamiltonian and the three ansätze to the larger complex $\left[\mathrm{Pt}\left(\mathrm{C}_{6} \mathrm{Cl}_{5}\right)_{4}\right]^{-}$. We chose the position of $\mathrm{Pt}$ as the common gauge origin for the RKB-CGO and RMB-CGO approaches. The structure is taken from Ref. 29 and we employ COSMO with the default parameters. ${ }^{[146[147 / 223}$ BH\&HLYP,,$[163[1971198$ B3LYP,, 1971199 PBE0,$\stackrel{1711172}{ }$ PBE0-40HF, ${ }^{291171172}$ TPSSh, ${ }^{202}$ TPSS0, ${ }^{202203}$ CAM-B3LYP, ${ }^{204}$ CAM-QPT-00, ${ }^{205}$ CAM-QTP-02, ${ }^{206}$ HSE06, $207 \sqrt[209]{2 C-\omega P B E, ~} \stackrel{210}{2}$ and $\omega$ B97X-D ${ }^{[173}$ are considered. Fine grids (grid 4a) are used for the numerical integration of the XC parts. $\frac{174}{176}$ The uncontracted Dyall-VTZ basis ${ }^{\sqrt{224}}$ is combined with the decontracted pcS-2 basis. ${ }^{169}$ We use the universal (uncontracted) x2c-type fitting bases ${ }^{132 \mid 164}$ for the RI- $J$ approximation in the 2c calculations. ${ }^{126}$ Furthermore, the $\mathrm{x} 2 \mathrm{c}-\mathrm{QZVP}$ all-2c basis ${ }^{164}$ is applied together with the tailored auxiliary basis. ${ }^{1321164}$ An SCF threshold of $10^{-8} \mathrm{E}_{\mathrm{h}}$ is selected. In total, the number of basis functions in the spherical atomic orbital representation amounts to 3360 (Dyall-VTZunc/pcS-2-unc) and 3003 (x2c-QZVPall-2c, 4720 primitive functions). 
In Sec. 7, we study the three lanthanide molecules $\left[\mathrm{La}(\mathrm{OAr} *)_{3}\right]^{-},\left[\mathrm{Lu}\left(\mathrm{NR}_{2}\right)_{3}\right]^{-}$, and $\left[\mathrm{Lu}\left(\mathrm{OAr}^{*}\right)_{3}\right]^{-}\left(\mathrm{OAr}^{*}=2,6-\mathrm{Ad}_{2}-4-\mathrm{t}-\mathrm{Bu}-\mathrm{C}_{6} \mathrm{H}_{2} \mathrm{O}, \mathrm{Ad}=\right.$ adamantyl, $\mathrm{t}-\mathrm{Bu}=$ tert-butyl, $\mathrm{R}=$ $\mathrm{SiMe}_{3}$ with $\mathrm{Me}=$ methyl) of Ref. 8 using the structures provided therein. The g-tensors are calculated with the x2c-SVPall-2c basis set for the light elements ${ }^{[132}$ and the $\mathrm{x} 2 \mathrm{c}-$ TZVPPall$2 c^{\frac{132}{132}}$ or $\mathrm{x} 2 \mathrm{c}-\mathrm{QZVP}$ all-2c basis $\frac{164}{164}$ for $\mathrm{La}$ and Lu. The mSNSO-DLU-X2C Hamiltonian is applied and we employ the TPSS, ${ }^{192} \mathrm{r}^{2} \mathrm{SCAN},{ }^{1951196} \mathrm{PBE} 0, \stackrel{1711172}{ } \mathrm{PBE} 0-40 \mathrm{HF},{ }^{291171172} \mathrm{CAM}-$

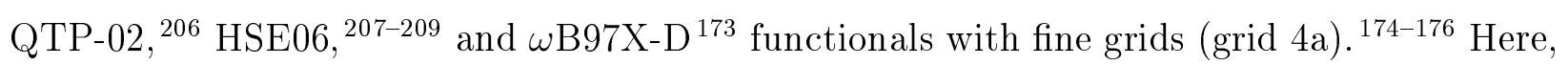
we apply COSMO to treat the molecular environment in solution. $[146[147$ Thus, we use the respective parameters for tetrahydrofuran (THF), which are $\epsilon_{r}=7.520$, a solvation radius of $1.30 \AA$, and a refractive index of 1.4050 . The SCF procedure is considered converged with an energy threshold of $10^{-7} \mathrm{E}_{\mathrm{h}}$ and criterion of $10^{-7}$ a.u. for the root mean square of the density matrix. The common gauge origin is placed at the La or Lu atom.

\section{Comparison to other Two-Component Approaches for Diatomic Molecules}

The $\Delta \mathrm{g}$ shifts of diatomic molecules at various two-component levels are shown in Tab. 2. Our gauge-invariant X2C framework is compared to the non-collinear DKH approach of Malkin et al. ${ }^{[27}$ and the ZORA Hamiltonian. ${ }^{[161}$ All 11 molecules feature a Kramers doublet ground state. Overall, the $\mathrm{X} 2 \mathrm{C}$ and DKH Hamiltonian lead to qualitatively similar results. For $\mathrm{HgH}$, the largest absolute deviations are observed with $14.6 \mathrm{ppt}\left(\Delta \mathrm{g}_{\perp}\right)$. The ZORA results show a larger deviation towards $\mathrm{X} 2 \mathrm{C}$ or DKH. Here, the deviation amounts to more than $60 \mathrm{ppt}$ for $\mathrm{PdH}$. This behavior can be rationalized by the closer formal relationship of $\mathrm{X} 2 \mathrm{C}$ and $\mathrm{DKH}$ as

well as the use of Gaussian-type orbitals. Note that the ZORA implementation ${ }^{231161}$ utilizes Slater-type orbitals, and thus the basis sets cannot be compared directly. Moreover, both the $\mathrm{X} 2 \mathrm{C}$ and DKH ansatz make use of three non-collinear calculations to assemble the complete g-tensor. Notably, this framework includes spin polarization. In contrast to DKH, magnetic 
Table 2: Principal components of the $\Delta \mathrm{g}$ shift in ppt for a set of diatomic molecules compiled in Ref. 161. Our gauge-invariant mSNSO-X2C approach is compared to the DKH ansatz with a common gauge origin of Ref. 27 and the ZORA formalism using GIAOs of Ref. 161. These corresponding results are taken from these references. Note that all calculations use tailored basis sets for the respective Hamiltonian and the BP86 functional. X2C results with the uncontracted Dyall-VTZ/pcS-2 basis and more sophisticated functionals are given in the Supporting Information. $\|$ and $\perp$ refer to the components of the g-tensor parallel and perpendicular to the bond axis. Experimental (Expt.) results are taken from Ref. 226.

\begin{tabular}{|c|c|c|c|c|c|c|c|c|}
\hline \multirow[b]{2}{*}{ Molecule } & \multicolumn{2}{|c|}{ X2C-GIAO } & \multicolumn{2}{|c|}{ DKH-CGO } & \multicolumn{2}{|c|}{ ZORA-GIAO } & \multicolumn{2}{|c|}{ Expt. } \\
\hline & $\Delta g_{\|}$ & $\Delta g_{\perp}$ & $\Delta g_{\|}$ & $\Delta g_{\perp}$ & $\Delta g_{\|}$ & $\Delta g_{\perp}$ & $\Delta g_{\|}$ & $\Delta g_{\perp}$ \\
\hline $\mathrm{RhC}$ & -3.0 & 53.6 & -0.6 & 56.9 & -2.0 & 50.3 & 1.6 & 51.8 \\
\hline $\mathrm{PdH}$ & -22.6 & 230.2 & -16.5 & 224.2 & -27.7 & 294.8 & -37.3 & 290.9 \\
\hline $\mathrm{ZnH}$ & -2.5 & -23.6 & -0.3 & -19.1 & -0.4 & -24.9 & -2.0 & -17.1 \\
\hline $\mathrm{CdH}$ & -4.1 & -62.1 & -1.9 & -59.8 & -2.4 & -71.7 & -5.3 & -49.9 \\
\hline $\mathrm{HgH}$ & -25.0 & -221.9 & -25.9 & -236.5 & -30.0 & -251.8 & -26.3 & -174.3 \\
\hline $\mathrm{ZnF}$ & -2.3 & -9.2 & -0.3 & -6.7 & -0.3 & -6.8 & -0.3 & -6.3 \\
\hline $\mathrm{CdF}$ & -2.7 & -22.4 & -0.8 & -21.2 & -0.9 & -21.4 & -1.3 & -17.3 \\
\hline $\mathrm{HgF}$ & -13.3 & -50.0 & -11.6 & -54.1 & -14.0 & -66.1 & -9.3 & -41.3 \\
\hline $\mathrm{ZnAg}$ & -2.2 & -20.6 & -0.2 & -19.0 & -0.3 & -21.8 & 0.2 & -11.8 \\
\hline $\mathrm{CdAg}$ & -3.1 & -46.9 & -1.1 & -45.9 & -1.3 & -51.3 & -0.9 & -31.2 \\
\hline $\mathrm{HgAg}$ & -10.7 & -131.8 & -10.0 & -140.9 & -10.4 & -143.8 & -6.5 & -88.7 \\
\hline
\end{tabular}

properties can be formulated straightforwardly in X2C. For instance, a back transformation of the DKH wave function to the 4c picture is applied to (approximately) arrive at the DKHtransformed expression of the first-order Zeeman operator in Ref. 27. A detailed discussion of magnetic fields in DKH and the associated difficulties is provided in Ref. 225.

All calculations utilize the generalized gradient approximation (GGA) functional BP86 and therefore a comparison with the experimental findings reveals notable deviations for some molecules. For instance, all methods fail to predict the correct sign of the $\Delta \mathrm{g}_{\|}$shift for RhC. Also for PdH, HgH, and $\mathrm{HgAg}$, the experimental results are not accurately reproduced. As shown by further studies in the Supporting Information, this can be reduced by choosing more sophisticated functionals such as PBE0 and $\omega$ B97X-D, while the uncontracted DyallVTZ/pcS-2 bases lead to very similar results. Further improvements are obtained when optimizing the structure at the same level of theory as for the calculation of the g-tensor. 
Especially for $\mathrm{PdH}$, this protocol results in a major improvement. The components of the gtensor change to $-33.2 \mathrm{ppt}$ and $315.4 \mathrm{ppt}$, which is much closer to the experimental findings of $-37.3 \mathrm{ppt}$ and $290.9 \mathrm{ppt}$.

\section{Assessment for Transition-Metal Complexes}

In this section, we first assess the accuracy of the relativistic Hamiltonian in Sec. 6.1. Then, the importance of the basis set is discussed in Sec. 6.2. Lastly, various density functional approximations are considered in Sec. 6.3. We only discuss the RMB-GIAO approach for the basis set and the density functional approximation in the main text. We refer to the Supporting Information for the other approaches.

\subsection{Comparison to Four-Component Data and Hamiltonian Studies}

The X2C and DLU-X2C Hamiltonian are further assessed by comparison to the 4c Hamiltonian for the 17 transition-metal complexes in Tab. 3. First, the quasi-relativistic Hamiltonians accurately reproduce the $4 \mathrm{c}$ results. Even for the W, Re, and Os compounds, the deviation of the isotropic $\Delta \mathrm{g}$ shift towards the $4 \mathrm{c}$ results amounts to at most $4 \mathrm{ppt}$. For the Mo and Tc complexes, the deviation is below 2 ppt and often even below 1 ppt. Similar trends are observed for the three principal components. We discuss the impact of the mSNSO approach on the results in detail in the Supporting Information. Here, we just state that it results in a substantial improvement. Generally, the calculated g-tensors are in good agreement with the experimental findings for the Mo, W, and Os complexes. Here, the deviation amounts to at most $10 \mathrm{ppt}$ for $\left[\mathrm{MoOF}_{4}\right]^{-}$and $15 \mathrm{ppt}$ for both $\left[\mathrm{WOBr}_{5}\right]^{2-}$ and $\left[\mathrm{OsOF}_{5}\right]$. Given the absolute value of the isotropic $\Delta \mathrm{g}$ shift for these complexes the Dyall-TZ/IGLOIII basis set and the PBE0 functional may already be considered sufficient. However, larger deviations are observed for the Re complexes with $60 \mathrm{ppt}$ for $\left[\mathrm{ReOBr}_{4}\right]$ and about $80 \mathrm{ppt}$ for $\left[\mathrm{ReOF}_{5}\right]^{-}$. These are errors of about $60 \%$ for $\left[\mathrm{ReOBr}_{4}\right]$ and $30 \%$ for $\left[\mathrm{ReOF}_{5}\right]^{-}$. 
Table 3: Principal components of the g-tensor relative to that of the free electron in ppt. Noncollinear 4c results are taken from the Supporting Information of Ref. 29. "PCC" denotes that the derivatives of $\boldsymbol{X}$ and $\boldsymbol{R}$ are neglected. Experimental (Expt.) results ${ }^{211}$ were collected in Ref. 29. All calculations utilize a common gauge origin and the RKB condition. Results with the Sapporo-DKH3-QZP-2012-diffuse bases are listed in the Supporting Information.

\begin{tabular}{|c|c|c|c|c|c|c|c|c|c|}
\hline Molecule & Hamiltonian & $\Delta g_{\text {iso }}$ & $\Delta g_{\|}$ & $\Delta g_{\perp}$ & Molecule & Hamiltonian & $\Delta g_{\text {iso }}$ & $\Delta g_{\|}$ & $\Delta g_{\perp}$ \\
\hline \multirow[t]{6}{*}[\mathrm{MoNCl}_{4}]{$^{2-}$} & X2C RKB PCC & -43.4 & -88.9 & -20.7 & \multirow[t]{6}{*}[\mathrm{TcNF}_{4}]{$^{-}$} & X2C RKB PCC & -41.5 & -77.6 & -23.4 \\
\hline & $\mathrm{X} 2 \mathrm{C} \mathrm{RKB}$ & -43.9 & -89.5 & -21.1 & & X2C RKB & -42.0 & -78.3 & -23.8 \\
\hline & DLU RKB PCC & -43.4 & -88.9 & -20.8 & & DLU RKB PCC & -41.5 & -77.7 & -23.4 \\
\hline & DLU RKB & -44.0 & -89.5 & -21.3 & & DLU RKB & -42.1 & -78.4 & -23.9 \\
\hline & 4c RKB & -43 & -90 & -20 & & 4c RKB & -41 & -78 & -23 \\
\hline & Expt. & -44 & -96 & -18 & & Expt. & -44 & -107 & -12 \\
\hline \multirow[t]{6}{*}[\mathrm{MoOF}_{4}]{$^{-}$} & X2C RKB PCC & -75.9 & -94.2 & -66.8 & \multirow[t]{6}{*}[\mathrm{TcNCl}_{4}]{$^{-}$} & X2C RKB PCC & 5.7 & 26.2 & -4.6 \\
\hline & X2C RKB & -76.4 & -94.8 & -67.2 & & X2C RKB & 5.2 & 25.5 & -4.9 \\
\hline & DLU RKB PCC & -75.9 & -94.1 & -66.9 & & DLU RKB PCC & 5.7 & 26.2 & -4.5 \\
\hline & DLU RKB & -76.6 & -94.9 & -67.4 & & DLU RKB & 5.2 & 25.6 & -5.0 \\
\hline & 4c RKB & -77 & -96 & -67 & & 4c RKB & 7 & 27 & -3 \\
\hline & Expt. & -87 & -108 & -77 & & Expt. & 0 & 6 & -2 \\
\hline \multirow[t]{6}{*}[\mathrm{MoOCl}_{4}]{$^{-}$} & $\mathrm{X} 2 \mathrm{C}$ RKB PCC & -42.3 & -16.9 & -55.0 & \multirow[t]{6}{*}[\mathrm{TcNBr}_{4}]{$^{-}$} & $\mathrm{X} 2 \mathrm{C}$ RKB PCC & 79.6 & 178.4 & 30.1 \\
\hline & X2C RKB & -42.7 & -17.5 & -55.4 & & X2C RKB & 79.0 & 177.7 & 29.7 \\
\hline & DLU RKB PCC & -42.4 & -16.9 & -55.1 & & DLU RKB PCC & 79.6 & 178.5 & 30.2 \\
\hline & DLU RKB & -42.9 & -17.6 & -55.6 & & DLU RKB & 78.9 & 177.6 & 29.5 \\
\hline & 4c RKB & -42 & -17 & -55 & & 4c RKB & 81 & 180 & 31 \\
\hline & Expt. & -49 & -37 & -56 & & Expt. & 69 & 145 & 32 \\
\hline \multirow{6}{*}[\mathrm{MoOF}_{5}]{$^{2-}$} & X2C RKB PCC & -104.5 & -101.6 & -105.9 & \multirow[t]{6}{*}[\mathbf{ReNF}_{4}]{$^{-}$} & X2C RKB PCC & -188.5 & -329.0 & -118.2 \\
\hline & X2C RKB & -104.9 & -102.2 & -106.3 & & X2C RKB & -189.2 & -330.1 & -118.8 \\
\hline & DLU RKB PCC & -104.5 & -101.5 & -105.9 & & DLU RKB PCC & -188.4 & -328.8 & -118.1 \\
\hline & DLU RKB & -105.1 & -102.3 & -106.5 & & DLU RKB & -189.4 & -330.1 & -119.0 \\
\hline & 4c RKB & -106 & -104 & -108 & & 4c RKB & -186 & -326 & -116 \\
\hline & Expt. & -104 & -128 & -91 & & Expt. & -206 & -353 & -132 \\
\hline \multirow[t]{6}{*}[\mathrm{MoOBr}_{5}]{$^{2-}$} & $\mathrm{X} 2 \mathrm{C}$ RKB PCC & -7.2 & 103.8 & -62.7 & \multirow[t]{6}{*}[\mathrm{ReNCl}_{4}]{$^{-}$} & $\mathrm{X} 2 \mathrm{C}$ RKB PCC & -75.2 & -72.7 & -76.4 \\
\hline & $\mathrm{X} 2 \mathrm{C} \mathrm{RKB}$ & -7.7 & 103.1 & -63.1 & & $\mathrm{X} 2 \mathrm{C} \mathrm{RKB}$ & -76.0 & -73.8 & -77.0 \\
\hline & DLU RKB PCC & -7.4 & 103.7 & -62.9 & & DLU RKB PCC & -75.1 & -72.7 & -76.3 \\
\hline & DLU RKB & -8.2 & 102.7 & -63.7 & & DLU RKB & -75.9 & -73.7 & -77.0 \\
\hline & 4c RKB & -7 & 104 & -63 & & 4c RKB & -74 & -72 & -74 \\
\hline & Expt. & -9 & 87 & -57 & & Expt. & -78 & -87 & -73 \\
\hline \multirow{6}{*}[\mathrm{WOCl}_{4}]{$^{-}$} & $\mathrm{X} 2 \mathrm{C}$ RKB PCC & -201.2 & -180.8 & -211.4 & \multirow[t]{6}{*}[\mathbf{ReNBr}_{4}]{$^{-}$} & X2C RKB PCC & 10.1 & 109.3 & -39.5 \\
\hline & X2C RKB & -201.9 & -181.8 & -212.0 & & X2C RKB & 9.2 & 108.1 & -40.2 \\
\hline & DLU RKB PCC & -201.1 & -180.7 & -211.3 & & DLU RKB PCC & 10.2 & 109.3 & -39.4 \\
\hline & DLU RKB & -201.9 & -181.7 & -211.9 & & DLU RKB & 9.1 & 108.0 & -40.4 \\
\hline & 4c RKB & -198 & -179 & -208 & & 4c RKB & 11 & 109 & -38 \\
\hline & Expt. & -229 & -209 & -239 & & Expt. & 3 & 67 & -29 \\
\hline \multirow[t]{6}{*}[\mathrm{WOF}_{5}]{$^{2-}$} & $\mathrm{X} 2 \mathrm{C}$ RKB PCC & -391.5 & -455.8 & -359.4 & \multirow[t]{6}{*}[\mathbf{ReOBr}_{4}]{} & $\mathrm{X} 2 \mathrm{C} \mathrm{RKB} \mathrm{PCC}$ & -39.4 & 230.8 & -174.5 \\
\hline & $\mathrm{X} 2 \mathrm{C} \mathrm{RKB}$ & -392.2 & -456.9 & -359.9 & & $\mathrm{X} 2 \mathrm{C} \mathrm{RKB}$ & -40.2 & 229.7 & -175.2 \\
\hline & DLU RKB PCC & -391.4 & -455.7 & -359.3 & & DLU RKB PCC & -39.3 & 230.9 & -174.4 \\
\hline & DLU RKB & -392.4 & -457.0 & -360.1 & & DLU RKB & -40.3 & 229.5 & -175.3 \\
\hline & 4c RKB & -388 & -451 & -356 & & 4c RKB & -37 & 231 & -172 \\
\hline & Expt. & -368 & -443 & -330 & & Expt. & -98 & 171 & -232 \\
\hline \multirow[t]{6}{*}[\mathbf{WOBr}_{5}]{$^{2-}$} & $\mathrm{X} 2 \mathrm{C}$ RKB PCC & -190.3 & -82.1 & -244.5 & \multirow[t]{6}{*}[\mathbf{ReOF}_{5}]{$^{-}$} & $\mathrm{X} 2 \mathrm{C}$ RKB PCC & -351.5 & -317.8 & -368.4 \\
\hline & $\mathrm{X} 2 \mathrm{C}$ RKB & -191.1 & -83.2 & -245.1 & & $\mathrm{X} 2 \mathrm{C}$ RKB & -352.3 & -318.9 & -369.0 \\
\hline & DLU RKB PCC & -190.3 & -82.1 & -244.4 & & DLU RKB PCC & -351.4 & -317.6 & -368.4 \\
\hline & DLU RKB & -191.5 & -83.5 & -245.4 & & DLU RKB & -352.4 & -318.9 & -369.2 \\
\hline & $4 c$ RKB & -187 & -81 & -240 & & 4c RKB & -348 & -314 & -365 \\
\hline & Expt. & -172 & -99 & -206 & & Expt. & -269 & -282 & -262 \\
\hline \multirow[t]{6}{*}[\mathrm{OsOF}_{5}]{} & X2C RKB PCC & -312.2 & -182.7 & -376.9 & & & & & \\
\hline & X2C RKB & -313.0 & -183.8 & -377.5 & & & & & \\
\hline & DLU RKB PCC & -312.1 & -182.6 & -376.9 & & & & & \\
\hline & DLU RKB & -313.1 & -183.8 & -377.7 & & & & & \\
\hline & 4c RKB & -309 & -180 & -374 & & & & & \\
\hline & Expt. & -324 & -197 & -387 & & & & & \\
\hline
\end{tabular}


Second, the error introduced by the DLU scheme is negligible. For the Mo and Tc complexes, the error is typically about $0.1 \mathrm{ppt}$. The maximum error is $0.4 \mathrm{ppt}$ for the isotropic $\Delta \mathrm{g}$ shift of $\left[\mathrm{WOBr}_{5}\right]^{2-}$, compared to an absolute value of $190 \mathrm{ppt}$. The errors of the principal components are very similar. Thus, no error cancellation is observed and the DLU scheme is a robust approximation. This confirms our previous findings for the HFC constant. $\stackrel{73}{73}$

Third, the impact of the decoupling derivatives typically amounts to about $1 \mathrm{ppt}$ for the heavier compounds. This may validate the previous $\mathrm{X} 2 \mathrm{C}$ implementation for the EPR g-tensor in Ref. 77. Notably, the error introduced by neglecting the decoupling derivatives is clearly larger than the error introduced by the local approximation and the derivatives can therefore routinely be included in DLU-X2C calculations with almost no computational overhead compared to a DLU-X2C energy calculation. For these complexes, the calculation of the g-tensor with the DLU-X2C Hamiltonian including derivatives takes a few seconds only. The computational costs are considered in detail in Sec. 6.4.

The three different approaches to the EPR g-tensor, i.e. RKB-CGO, RMB-CGO, and RMB-GIAO, are compared in Tab. 4. The DLU error is negligible in all cases, which further demonstrates the broad applicability of this approximation. Overall, the impact of the RMB and the GIAOs on the actual results is comparably small. As expected, the largest absolute deviations are found for the heavy $\mathrm{W}, \mathrm{Re}$, and Os compounds. There, deviations of about 2-3 ppt are observed. For $\left[\mathrm{ReNBr}_{4}\right]^{-}$, this amounts to a relative change of about $17 \%$, while it amounts to only $1 \%$ for $\left[\mathrm{ReOF}_{5}\right]^{-}$. The small impact of the GIAOs can be rationalized by analyzing the individual terms for the EPR g-tensor in Eq. 28. The contribution of the one-electron potential and the two-electron integrals compensate each other to a large extent. The main advantage of GIAOs is that the working equations become gauge-origin invariant. The price to pay for this physical constraint is the evaluation of the two-electron integralsespecially the HF exchange integrals. For instance, the calculation of the g-tensor of $\left[\mathrm{OsOF}_{5}\right]$ with 499 basis functions (spherical atomic orbital representation) takes about 8.8 minutes with the RMB-GIAO ansatz. Here, the computation of the one-electron terms requires 6 
Table 4: Comparison of two-component Hamiltonians for the principal components of the $\Delta$ g-tensor in ppt. Experimental (Expt.) results ${ }^{211} 222$ were collected in Ref. 29.

\begin{tabular}{|c|c|c|c|c|c|c|c|c|c|}
\hline Molecule & Hamiltonian & $\Delta g_{\text {iso }}$ & $\Delta g_{\|}$ & $\Delta g_{\perp}$ & Molecule & Hamiltonian & $\Delta g_{\text {iso }}$ & $\Delta g_{\|}$ & $\Delta g_{\perp}$ \\
\hline \multirow[t]{7}{*}[\mathrm{MoNCl}_{4}]{$^{2-}$} & X2C RKB & -43.9 & -89.5 & -21.1 & \multirow[t]{7}{*}[\mathrm{TcNF}_{4}]{$^{-}$} & X2C RKB & -42.0 & -78.3 & -23.8 \\
\hline & $\mathrm{X} 2 \mathrm{C} \mathrm{RMB}$ & -43.2 & -88.8 & -20.4 & & $\mathrm{X} 2 \mathrm{C} \mathrm{RMB}$ & -41.2 & -77.5 & -23.1 \\
\hline & X2C GIAO & -42.4 & -88.0 & -19.7 & & X2C GIAO & -40.8 & -76.6 & -22.9 \\
\hline & DLU RKB & -44.0 & -89.5 & -21.3 & & DLU RKB & -42.1 & -78.4 & -23.9 \\
\hline & DLU RMB & -43.2 & -88.8 & -20.5 & & DLU RMB & -41.3 & -77.6 & -23.1 \\
\hline & DLU GIAO & -42.5 & -88.0 & -19.8 & & DLU GIAO & -40.9 & -76.8 & -22.9 \\
\hline & Expt. & -44 & -96 & -18 & & Expt. & -44 & -107 & -12 \\
\hline \multirow{7}{*}[\mathrm{MoOF}_{4}]{$^{-}$} & X2C RKB & -76.4 & -94.8 & -67.2 & \multirow[t]{7}{*}[\mathrm{TcNCl}_{4}]{$^{-}$} & X2C RKB & 5.2 & 25.5 & -4.9 \\
\hline & X2C RMB & -75.7 & -94.0 & -66.5 & & X2C RMB & 5.9 & 26.3 & -4.2 \\
\hline & X2C GIAO & -75.2 & -93.3 & -66.1 & & X2C GIAO & 6.5 & 27.4 & -3.9 \\
\hline & DLU RKB & -76.6 & -94.9 & -67.4 & & DLU RKB & 5.2 & 25.6 & -5.0 \\
\hline & DLU RMB & -75.7 & -94.0 & -66.6 & & DLU RMB & 5.9 & 26.2 & -4.2 \\
\hline & DLU GIAO & -75.2 & -93.4 & -66.2 & & DLU GIAO & 6.5 & 27.4 & -3.9 \\
\hline & Expt. & -87 & -108 & -77 & & Expt. & 0 & 6 & -2 \\
\hline \multirow[t]{7}{*}[\mathrm{MoOCl}_{4}]{$^{-}$} & X2C RKB & -42.7 & -17.5 & -55.4 & \multirow[t]{7}{*}[\mathrm{TcNBr}_{4}]{$^{-}$} & X2C RKB & 79.0 & 177.7 & 29.7 \\
\hline & X2C RMB & -42.0 & -16.8 & -54.7 & & X2C RMB & 79.9 & 178.6 & 30.6 \\
\hline & X2C GIAO & -41.4 & -15.9 & -54.2 & & X2C GIAO & 80.6 & 179.3 & 31.3 \\
\hline & DLU RKB & -42.9 & -17.6 & -55.6 & & DLU RKB & 78.9 & 177.6 & 29.5 \\
\hline & DLU RMB & -42.1 & -16.8 & -54.8 & & DLU RMB & 79.9 & 178.6 & 30.6 \\
\hline & DLU GIAO & -41.5 & -15.9 & -54.3 & & DLU GIAO & 80.6 & 179.3 & 31.2 \\
\hline & Expt. & -49 & -37 & -56 & & Expt. & 69 & 145 & 32 \\
\hline \multirow{7}{*}[\mathrm{MoOF}_{5}]{$^{2-}$} & X2C RKB & -104.9 & -102.2 & -106.3 & \multirow[t]{7}{*}[\mathrm{ReNF}_{4}]{$^{-}$} & X2C RKB & -189.2 & -330.1 & -118.8 \\
\hline & X2C RMB & -104.2 & -101.4 & -105.5 & & X2C RM & -188.7 & -329.5 & -118.3 \\
\hline & X2C GIAO & -103.4 & -100.5 & -104.9 & & X2C GIAO & -187.2 & -326.5 & -117.5 \\
\hline & DLU RKB & -105.1 & -102.3 & -106.5 & & DLU RKB & -189.4 & -330.1 & -119.0 \\
\hline & DLU RMB & -104.2 & -101.4 & -105.6 & & DLU RMB & -188.7 & -329.5 & -118.3 \\
\hline & DLU GIAO & -103.5 & -100.6 & -104.9 & & DLU GIAO & -187.2 & -326.5 & -117.5 \\
\hline & Expt. & -104 & -128 & -91 & & Expt. & -206 & -353 & -132 \\
\hline \multirow{7}{*}[\mathrm{MoOBr}_{5}]{$^{2-}$} & $\mathrm{X} 2 \mathrm{C}$ RKB & -7.7 & 103.1 & -63.1 & \multirow{7}{*}[\mathrm{ReNCl}_{4}]{$^{-}$} & $\mathrm{X} 2 \mathrm{C} \mathrm{RKB}$ & -76.0 & -73.8 & -77.0 \\
\hline & $\mathrm{X} 2 \mathrm{C} \mathrm{RMB}$ & -6.7 & 104.1 & -62.1 & & X2C RMB & -75.5 & -73.3 & -76.6 \\
\hline & X2C GIAO & -6.5 & 104.7 & -62.1 & & X2C GIAO & -74.5 & -72.2 & -75.6 \\
\hline & DLU RKB & -8.2 & 102.7 & -63.7 & & DLU RKB & -75.9 & -73.7 & -77.0 \\
\hline & DLU RMB & -7.0 & 104.0 & -62.4 & & DLU RMB & -75.5 & -73.4 & -76.6 \\
\hline & DLU GIAO & -6.7 & 104.6 & -62.4 & & DLU GIAO & -74.4 & -72.2 & -75.6 \\
\hline & Expt. & -9 & 87 & -57 & & Expt. & -78 & -87 & -73 \\
\hline \multirow{7}{*}[\mathrm{WOCl}_{4}]{$^{-}$} & X2C RKB & -201.9 & -181.8 & -212.0 & \multirow{7}{*}[\operatorname{ReNBr}_{4}]{$^{-}$} & $\mathrm{X} 2 \mathrm{C} \mathrm{RKB}$ & 9.2 & 108.1 & -40.2 \\
\hline & X2C RMB & -201.4 & -181.3 & -211.5 & & X2C RMB & 9.8 & 108.7 & -39.7 \\
\hline & X2C GIAO & -200.6 & -180.4 & -210.6 & & X2C GIAO & 10.8 & 110.7 & -39.1 \\
\hline & DLU RKB & -201.9 & -181.7 & -211.9 & & DLU RKB & 9.1 & 108.0 & -40.4 \\
\hline & DLU RMB & -201.4 & -181.3 & -211.5 & & DLU RMB & 9.8 & 108.6 & -39.6 \\
\hline & DLU GIAO & -200.5 & -180.4 & -210.6 & & DLU GIAO & 10.8 & 110.7 & -39.1 \\
\hline & Expt. & -229 & -209 & -239 & & Expt. & 3 & 67 & -29 \\
\hline$\left[\mathbf{W O F}_{5}\right]^{2-}$ & X2C RKB & -392.2 & -456.9 & -359.9 & {$\left[\mathrm{ReOBr}_{4}\right]$} & $\mathrm{X} 2 \mathrm{C} \mathrm{RKB}$ & -40.2 & 229.7 & -175.2 \\
\hline & X2C RMB & -391.7 & -456.3 & -359.4 & & X2C RMB & -39.7 & 230.3 & -174.6 \\
\hline & X2C GIAO & -389.2 & -451.8 & -358.0 & & X2C GIAO & -39.3 & 230.7 & -174.2 \\
\hline & DLU RKB & -392.4 & -457.0 & -360.2 & & DLU RKB & -40.3 & 229.5 & -175.3 \\
\hline & DLU RMB & -391.8 & -456.4 & -359.4 & & DLU RMB & -39.7 & 230.2 & -174.6 \\
\hline & DLU GIAO & -389.3 & -451.8 & -358.0 & & DLU GIAO & -39.2 & 230.7 & -174.2 \\
\hline & Expt. & -368 & -443 & -330 & & Expt. & -98 & 171 & -232 \\
\hline$\left[\mathbf{W O B r}_{5}\right]^{2-}$ & $\mathrm{X} 2 \mathrm{C}$ RKB & -191.1 & -83.2 & -245.1 & {$\left[\mathbf{R e O F}_{5}\right]^{-}$} & $\mathrm{X} 2 \mathrm{C}$ RKB & -352.3 & -318.9 & -369.0 \\
\hline & X2C RMB & -190.5 & -82.6 & -244.5 & & X2C RMB & -351.7 & -318.3 & -368.5 \\
\hline & X2C GIAO & -190.1 & -79.3 & -245.5 & & X2C GIAO & -350.3 & -315.1 & -367.8 \\
\hline & DLU RKB & -191.5 & -83.5 & -245.4 & & DLU RKB & -352.4 & -318.9 & -369.2 \\
\hline & DLU RMB & -190.6 & -82.7 & -244.5 & & DLU RMB & -351.8 & -318.3 & -368.5 \\
\hline & DLU GIAO & -190.1 & -79.3 & -245.5 & & DLU GIAO & -350.3 & -315.1 & -367.8 \\
\hline & Expt. & -172 & -99 & -206 & & Expt. & -269 & -282 & -262 \\
\hline$\left[\mathrm{OsOF}_{5}\right]$ & $\mathrm{X} 2 \mathrm{C}$ RKB & -313.0 & -183.8 & -377.5 & & & & & \\
\hline & $\mathrm{X} 2 \mathrm{C} \mathrm{RMB}$ & -312.4 & -183.3 & -376.9 & & & & & \\
\hline & X2C GIAO & -311.8 & -181.7 & -376.8 & & & & & \\
\hline & DLU RKB & -313.1 & -183.8 & -377.7 & & & & & \\
\hline & DLU RMB & -312.4 & -183.2 & -376.9 & & & & & \\
\hline & DLU GIAO & -311.8 & -181.7 & -376.8 & & & & & \\
\hline & Expt. & -324 & -197 & -387 & & & & & \\
\hline
\end{tabular}


seconds. In contrast, only 4 and 5 seconds are required for the RKB-CGO and RMB-CGO approach, respectively. Timings are measured with the DLU-X2C Hamiltonian and a single thread of an Intel ${ }^{\circledR}$ Xeon ${ }^{\circledR}$ Gold $6212 \mathrm{U}$ CPU @ 2.40 GHz. For this purpose, the code was compiled with Intel ${ }^{\circledR}$ Fortran Compiler 19.0.1.144. Compared to the SCF procedure, this computational effort is negligible with all approaches for the g-tensor.

\subsection{Basis Set Study}

The results of the relativistic all-electron basis set study on the 15 aforementioned transitionmetal complexes are summarized in Figure 1. For each basis set, the mean absolute percentwise deviation (MAPD) and the corresponding standard deviation (STD) were computed over the sum of the 15 compounds. The MAPD is calculated as follows:

$$
\operatorname{MAPD}=\sum_{i=1}^{N=15} \frac{\left|\Delta g_{i, \text { iso }}^{\mathrm{test}}-\Delta g_{i, \text { iso }}^{\mathrm{ref}}\right|}{\left|\Delta g_{i, \text { iso }}^{\mathrm{ref}}\right|}
$$

where $\Delta g_{i, \text { iso }}^{\text {test }}$ and $\Delta g_{i, \text { iso }}^{\text {ref }}$ denote the isotropic g-shift for the test basis set and the reference basis set, respectively.

From the figure, it is clear that the Sapporo-type basis sets perform consistently worse than the others included in this study, which starkly contrasts with its good performance for the HFC constant observed in our previous work for the same set of compounds. ${ }^{[3]}$ Even the quadruple- $\zeta$ analog of the Sapporo basis sets results in an MAPD and STD of over 9.32\%, which is far larger than that of the pcS-3/Dyall-VQZ (1.10\%), cc-pVQZ/Dyall-VQZ (1.80\%), and x2c-QZVPall-2c (1.98\%) configurations. Thus, the results are more similar to the NMR shielding studies, in which the x2c-type and Dyall bases also outperformed the Sapporo basis sets. $164 \mid 176$

Conversely, the triple- $\zeta$ quality basis sets aside from the Sapporo-DKH3-TZP-2012 configurations achieve accurate results when compared to the reference values, with MAPDs of less than $3 \%$. In particular, going to the quadrupole- $\zeta$ quality analog did not significantly 
Figure 1: Assessment of various basis sets compared to an even-tempered reference for 15 of the 17 transition-metal complexes. $\left[\mathrm{TcNCl}_{4}\right]^{-}$is omitted due to a small absolute $\Delta \mathrm{g}$ shift (4 ppt) and $\left[\mathrm{WOF}_{5}\right]^{2-}$ is neglected in the statistical evaluation due to convergence issues for the alignment of spin $x$ and $y$ with the ET basis. MAPD and STD denote the mean absolute percent-wise error and its standard deviation. For brevity, the suffix "unc" is omitted for the combinations of the Dyall basis and the ANO basis sets. See Tab. 1 for further details on the basis sets and their size. Note that we excluded the Jorge basis sets due to large errors of more than $90 \%$.

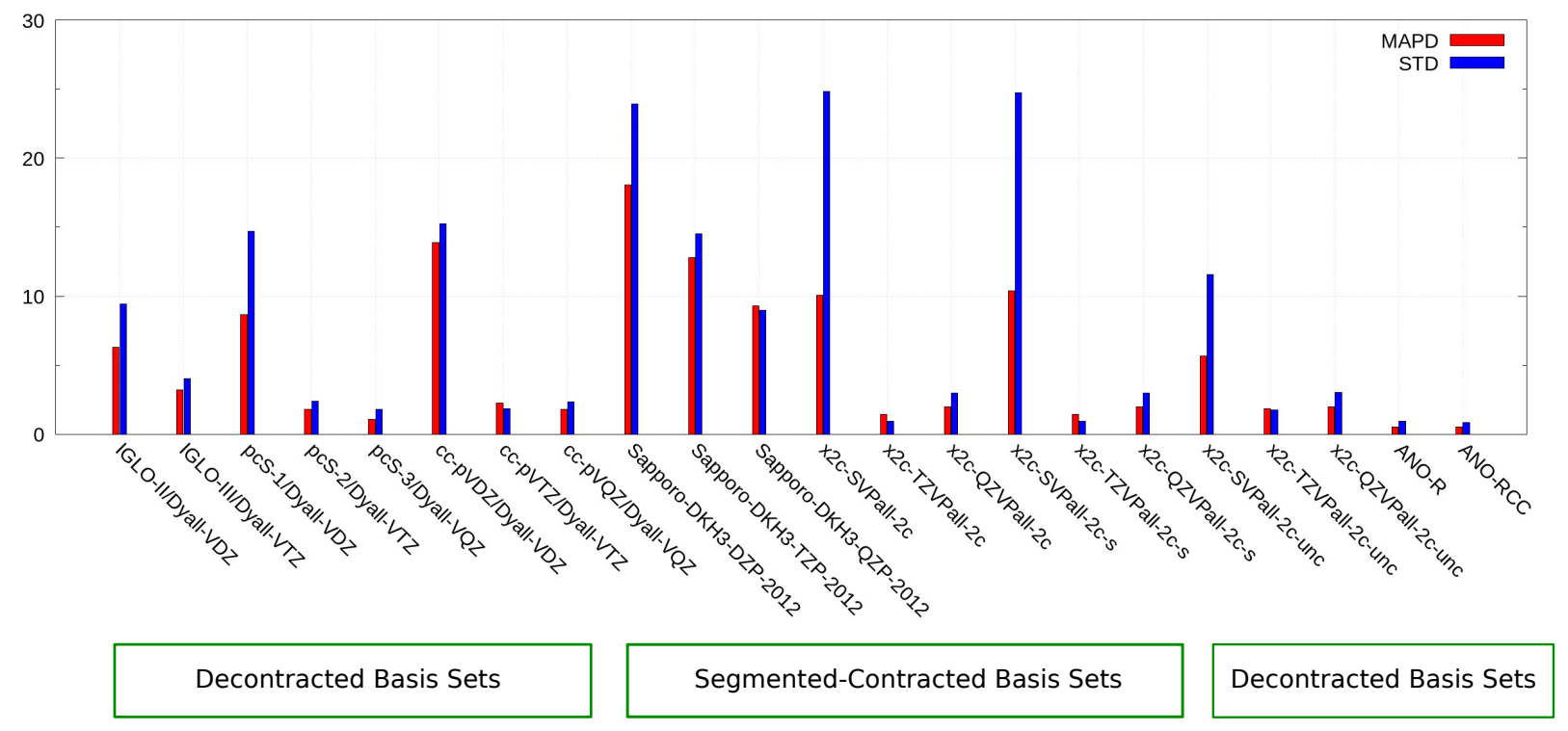

change the MAPD and STD for all basis sets. This also holds true when comparing the decontracted and contracted x2c-XZVPall-2c $(\mathrm{X}=\mathrm{T}, \mathrm{Q})$ basis sets. The ANO-R and ANO-RCC basis sets achieve the lowest MAPD and STD of all configurations studied, at 0.52/0.94 and $0.53 / 0.87$, respectively.

In summary, these results indicate that triple- $\zeta$ quality basis sets are sufficient for accurate calculations of the g-tensor when using the cc-pVTZ/Dyall-VTZ, x2c-TZVPall-2c, and x2cTZVPall-2c-s basis sets for these species. In particular, quadrupole- $\zeta$ or even decontracted basis sets are unnecessary if the x2c-TZVPall-2c basis set is used, which significantly reduces the total basis set dimension (see also Tab. 1). The quadruple- $\zeta$ basis sets may be used for benchmark calculations. 


\subsection{Study of Density Functional Approximations}

The impact of frequently used density functionals of various rungs is shown in Fig. 2 for the

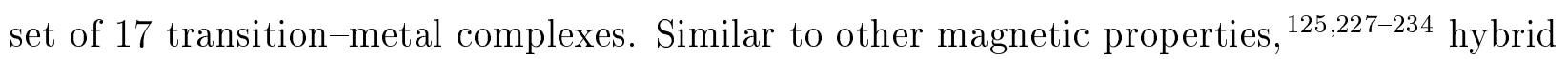
functionals usually lead to an improvement over pure density functionals. Yet, the errors of the hybrid functionals are systematically larger than those found previously for the EPR HFC constant. Specifically, the errors for the g-tensor range from roughly $10 \%$ to less than $30 \%$, whereas errors for the HFC constant span from $5 \%$ to less than $25 \% \cdot \frac{.73}{}$

Considering the Laplacian or the (generalized) kinetic energy in the functional approximation does not reduce the errors. Here, we observe just the opposite, and the performance of all meta-GGAs is notably inferior to that of GGAs. Particularly, the performance of $\mathrm{r}^{2} \mathrm{SCAN}$ deteriorates substantially, in contrast to the considerable improvement over GGAs that ${ }^{2}$ SCAN gave for HFC constants. ${ }^{[73}$

The errors of GGA hybrid functionals cover a range from 15\% to $20 \%$. The B97 family and PBE0 perform best within this class. Note that B97 and B3LYP performed poorly

Figure 2: Assessment of density functional approximations compared to the experimental findings for 15 of the 17 transition-metal complexes. $\left[\mathrm{ReNBr}_{4}\right]^{-}$and $\left[\mathrm{TcNCl}_{4}\right]^{-}$are neglected in the statistical evaluation. MAPD and STD denote the mean absolute percent-wise error and its standard deviation.




for the HFC constants. TPSSh and revTPSSh do not include a sufficient amount of HF exchange as shown by the reduced errors of TPSS0 with $25 \%$ of exchange instead of $10 \%$. The performance of the latter is on par with B3LYP and BH\&HLYP. Range-separation or the Coulomb attenuating method do not achieve consistent improvements; whereas CAMB3LYP reduced the errors compared to its parent B3LYP, CAM-QTP-00 shows the largest errors among all hybrid functionals. PBE0, LC- $\omega$ PBE, and $\omega$ B97X-D show very similar errors as for the HFC constant. The MAPDs amount to about $15 \%$ and we recommend these functionals for an error-balanced calculation of EPR spectra.

\subsection{Application to $\left[\mathrm{Pt}\left(\mathrm{C}_{6} \mathrm{Cl}_{5}\right)_{4}\right]^{-}$}

To illustrate the computational costs, we apply the X2C and the DLU-X2C Hamiltonian to $\left[\mathrm{Pt}\left(\mathrm{C}_{6} \mathrm{Cl}_{5}\right)_{4}\right]^{-}$, described by more than 3000 basis functions. The $\Delta \mathrm{g}$ shifts are listed in Tab. 5. In line with the previous study of the density functional approximations, we only consider hybrid density functionals.

Generally, the uncontracted Dyall-VTZ/pcS-2 and the segmented-contracted x2c-QZVPall2c basis sets lead to very similar results with the RKB and RMB condition using a common gauge origin as well as the RMB-GIAO ansatz. Notably, the magnetic-field derivatives of COSMO do not lead to substantial changes. We note that an improved relativistic description with COSMO formally requires further generalizations in two aspects. First, the parameters such as the solvation radii and the grids for the COSMO surface are not optimized for relativistic all-electron calculations. $\frac{146 \mid 147}{}$ Second, we do not apply the relativistic picture-change correction to COSMO. Such a correction is less straightforward to implement than the corresponding correction to expectation values since the COSMO surface is optimized in the SCF iterations. $\frac{146[147}{11}$

Overall, the CAM-QTP functionals perform remarkably well for the isotropic shift and the three principal components. Note that this performance of CAM-QTP-00 is in stark contrast to the findings in Sec. 6.3 and the accuracy of CAM-QTP-00 significantly depends 
Table 5: Principal components of the $\Delta \mathrm{g}$ shift for $\left[\mathrm{Pt}\left(\mathrm{C}_{6} \mathrm{Cl}_{5}\right)_{4}\right]^{-}$in ppt. RKB and RMB refer to restricted kinetic and magnetic balance, while $\mathrm{CGO}$ and GIAO denote a common gauge origin and gauge-including atomic orbitals, respectively. We use the mSNSO-DLU-X2C Hamiltonian. Experimental (Expt.) results are taken from Ref. 235.

\begin{tabular}{|c|c|c|c|c|c|c|c|c|c|}
\hline \multirow[b]{2}{*}{ Functional } & \multirow[b]{2}{*}{ Hamiltonian } & \multicolumn{4}{|c|}{ Dyall-VTZ-unc/pcS-2-unc } & \multicolumn{4}{|c|}{ x2c-QZVPall-2c } \\
\hline & & $\Delta \mathrm{g}_{\text {iso }}$ & $\Delta \mathrm{g}_{11}$ & $\Delta \mathrm{g}_{22}$ & $\Delta \mathrm{g}_{33}$ & $\Delta \mathrm{g}_{\text {iso }}$ & $\Delta \mathrm{g}_{11}$ & $\Delta \mathrm{g}_{22}$ & $\Delta \mathrm{g}_{33}$ \\
\hline \multirow[t]{3}{*}{ BH\&HLYP } & RKB CGO & 553 & -372 & 953 & 1078 & 549 & -374 & 949 & $\overline{1072}$ \\
\hline & RMB CGO & 554 & -371 & 954 & 1079 & 550 & -372 & 950 & 1072 \\
\hline & RMB GIAO & 553 & -371 & 953 & 1079 & 550 & -372 & 950 & 1072 \\
\hline \multirow[t]{3}{*}{ B3LYP } & RKB CGO & 425 & -409 & 648 & 1002 & 420 & -412 & 676 & 997 \\
\hline & RMB CGO & 427 & -408 & 685 & 1003 & 422 & -410 & 678 & 998 \\
\hline & RMB GIAO & 426 & -408 & 683 & 1004 & 422 & -410 & 678 & 998 \\
\hline \multirow[t]{3}{*}{ PBE0 } & RKB CGO & 462 & -350 & 787 & 948 & 454 & -354 & 779 & 938 \\
\hline & RMB CGO & 463 & -349 & 788 & 949 & 456 & -352 & 780 & 939 \\
\hline & RMB GIAO & 462 & -349 & 787 & 949 & 456 & -352 & 780 & 939 \\
\hline \multirow[t]{3}{*}{ PBE0-40HF } & RKB CGO & 513 & -337 & 888 & 988 & 510 & -341 & 885 & 986 \\
\hline & RMB CGO & 514 & -337 & 890 & 989 & 511 & -339 & 886 & 987 \\
\hline & RMB GIAO & 513 & -337 & 888 & 989 & 511 & -339 & 886 & 987 \\
\hline \multirow[t]{3}{*}{ TPSSh } & RKB CGO & 326 & -355 & 581 & 751 & 317 & -359 & 576 & 734 \\
\hline & RMB CGO & 327 & -354 & 582 & 752 & 319 & -357 & 578 & 736 \\
\hline & RMB GIAO & 333 & -351 & 588 & 762 & 325 & -354 & 585 & 745 \\
\hline \multirow[t]{3}{*}{ TPSS0 } & RKB CGO & 435 & -310 & 752 & 864 & 430 & -313 & 747 & 856 \\
\hline & RMB CGO & 436 & -309 & 753 & 865 & 430 & -313 & 747 & 856 \\
\hline & RMB GIAO & 441 & -307 & 759 & 873 & 437 & -310 & 755 & 865 \\
\hline \multirow[t]{3}{*}{ CAM-B3LYP } & RKB CGO & 535 & -397 & 919 & 1083 & 534 & -399 & 917 & 1084 \\
\hline & RMB CGO & 536 & -396 & 921 & 1084 & 535 & -398 & 918 & 1085 \\
\hline & RMB GIAO & 536 & -396 & 919 & 1084 & 535 & -398 & 918 & 1085 \\
\hline \multirow[t]{3}{*}{ CAM-QTP-00 } & RKB CGO & 601 & -382 & 1034 & 1150 & 603 & -384 & 1035 & 1158 \\
\hline & RMB CGO & 602 & -381 & 1036 & 1151 & 604 & -382 & 1036 & 1158 \\
\hline & RMB GIAO & 601 & -381 & 1034 & 1150 & 604 & -382 & 1036 & 1158 \\
\hline \multirow[t]{3}{*}{ CAM-QTP-02 } & RKB CGO & 614 & -413 & 1057 & 1198 & 609 & -416 & 1056 & 1186 \\
\hline & RMB CGO & 615 & -413 & 1058 & 1199 & 610 & -415 & 1057 & 1187 \\
\hline & RMB GIAO & 614 & -413 & 1056 & 1198 & 610 & -415 & 1057 & 1187 \\
\hline \multirow[t]{3}{*}{ HSE06 } & RKB CGO & 446 & -364 & 759 & 943 & 443 & -367 & 754 & 941 \\
\hline & RMB CGO & 447 & -363 & 760 & 945 & 444 & -365 & 755 & 942 \\
\hline & RMB GIAO & 447 & -363 & 758 & 945 & 444 & -365 & 755 & 943 \\
\hline \multirow[t]{3}{*}{ LC- $\omega$ PBE } & RKB CGO & 534 & -349 & 940 & 1011 & 531 & -353 & 938 & 1008 \\
\hline & RMB CGO & 535 & -348 & 942 & 1012 & 532 & -351 & 939 & 1008 \\
\hline & RMB GIAO & 534 & -348 & 940 & 1011 & 532 & -351 & 939 & 1009 \\
\hline \multirow[t]{3}{*}{$\omega \mathrm{B} 97 \mathrm{X}-\mathrm{D}$} & RKB CGO & 529 & -398 & 893 & 1093 & 528 & -401 & 891 & 1094 \\
\hline & RMB CGO & 530 & -397 & 894 & 1094 & 529 & -399 & 892 & 1095 \\
\hline & RMB GIAO & 530 & -397 & 893 & 1094 & 529 & -399 & 892 & 1095 \\
\hline Expt. & & 594 & -400 & 1005 & 1177 & 594 & -400 & 1005 & 1177 \\
\hline
\end{tabular}


on the molecule. $\omega \mathrm{B} 97 \mathrm{X}-\mathrm{D}$ reproduces one of the three components very well but loses some ground for the other components. Generally, range-separated functionals and Becke's half and half functional (BH\&HLYP) yield the most accurate results for this complex. This is in line with previous studies on the HFC constant $\stackrel{29 / 73}{ }$ and also similar to results for other magnetic properties.125/227-234 TPSSh and HSE06 are not sufficient as they fail to deliver accurate results for $\Delta g_{22}$ and $\Delta g_{33}$. Again, this may be explained by the fact that TPSSh only includes $10 \%$ of HF exchange. Consequently, TPSS0 significantly improves upon TPSSh. Moreover, we previously observed that the results with HSE06 deteriorate for the HFC tensor of larger molecules. $\frac{73}{3}$

We discuss the computational costs for the PBE0 functional. First, a two-component SCF calculation with the uncontracted Dyall-VTZ/pcS-2 basis set (3360 basis functions in the spherical representation) takes between 5.5 and 8 hours on 12 threads of an Intel ${ }^{\circledR}$ Xeon ${ }^{\circledR}$ Gold $6212 \mathrm{U}$ CPU @ 2.40 GHz. This requires 69-109 iterations. The calculation of the g-tensor with a common gauge origin only takes 17 seconds with the DLU scheme and the RKB condition. This is increased to 22 seconds with the RMB condition. The RMBGIAO approach requires 1.0 hours. Here, the one-electron terms need 47 seconds, while the computation of the Coulomb and the HF exchange integrals amounts to 57.5 minutes. The derivatives of the exchange-correlation energy are evaluated in 2 minutes. Thus, efficient and robust approximations to the HF exchange integrals such as an extension of the twocomponent seminumerical exchange approximation for SCF energies $\frac{130 \mid 131}{10}$ magnetic-field derivatives are desirable. Matters are similar for the x2c-QZVPall-2c basis set, which leads to 3003 basis functions in total (4720 primitive basis functions). Here, the SCF procedure requires 26.5 to 41.9 hours for $64-106$ SCF iterations, see also Ref. 73. The RKB-CGO and RMB-CGO calculations of the g-tensor take 1.0 and 1.5 minutes, respectively. This is increased to 4.2 hours with the RMB-GIAO ansatz, for which the one-electron parts need 2.7 minutes and the two-electron integrals require the remaining $98 \%$ of the computational time (4.1 hours). The RI- $J$ and the XC terms only take 2.5 and 7.1 minutes, respectively. 
Again, the HF exchange integrals dominate the computational costs for this approach. The acceleration of the DLU scheme is significant as the formal scaling of the one-electron Hamiltonian (derivatives) is reduced form $\mathcal{O}\left(N^{3}\right)$ to $\mathcal{O}\left(N^{2}\right)$, where $N$ measures the system-size. For the RKB-CGO and the RMB-CGO approach, the full X2C Hamiltonian needs 64.8 and 69.7 minutes, whereas the wall time for RMB-GIAO is 75 minutes. Therefore, the DLU scheme leads to a speed-up by a factor of 29-61 for the one-electron part and the maximum error for the principle components amounts to about $5 \mathrm{ppt}$ for both x2c-QZVPall-2c and the Dyall bases. The errors of isotropic the $\Delta \mathrm{g}$ shift are $1.5 \mathrm{ppt}$ and $2.2 \mathrm{ppt}$. The speed-up decreases from RKB-CGO to RMB-GIAO as more integral derivatives need to be evaluated and all blocks of the integrals are required for the DLU scheme to consider the interatomic off-diagonal corrections. For pure density functionals, the computation time of the full X2C Hamiltonian is much larger than that of the RI- $J$ integrals and the $\mathrm{XC}$ terms. Therefore, the $\mathrm{X} 2 \mathrm{C}$ step will dominate the corresponding computational costs for pure density functionals.

In conclusion, calculations of large molecules are possible with limited computational resources and the convergence of the SCF procedure is the time-determining part of the computational protocol. Therefore, the GIAO-RMB ansatz can be routinely used for largescale calculations.

\section{Application to Lanthanide Molecules}

We recently demonstrated that our implementation of the hyperfine coupling constant within the one-electron exact two-component theory framework accurately reproduces the experimental HFC constants of the three lanthanide complexes $\left[\mathrm{La}\left(\mathrm{OAr}^{*}\right)_{3}\right]^{-},\left[\mathrm{Lu}\left(\mathrm{NR}_{2}\right)_{3}\right]^{-}$, and $\left[\mathrm{Lu}\left(\mathrm{OAr}^{*}\right)_{3}\right]^{-}$of Ref. $\underline{8}$, which represent potential molecular qubit materials. ${ }^{73}$

To supplement this study and demonstrate that the spin Hamiltonian parameters for this class of important compounds can be accurately predicted by the present method, we calculate the g-tensors for the experimental HFC constants of the three lanthanide complexes 
Table 6: Principal components of the g-tensor for the three spin- $\frac{1}{2} \mathrm{La}(\mathrm{II})$ and $\mathrm{Lu}(\mathrm{II})$ molecules $\left.\left[\mathrm{La}\left(\mathrm{OAr}^{*}\right)_{3}\right]^{-}, \mathrm{Lu}\left(\mathrm{NR}_{2}\right)_{3}\right]^{-}$, and $\left[\mathrm{Lu}\left(\mathrm{OAr}^{*}\right)_{3}\right]^{-}\left(\mathrm{OAr}^{*}=2,6-\mathrm{Ad}_{2}-4-\mathrm{t}-\mathrm{Bu}-\mathrm{C}_{6} \mathrm{H}_{2} \mathrm{O}\right.$, $\mathrm{Ad}=$ adamantyl, $\mathrm{t}-\mathrm{Bu}=$ tert-butyl, $\mathrm{R}=\mathrm{SiMe}_{3}$ with $\mathrm{Me}=$ methyl).$\stackrel{8}{\mathrm{~B}} \mathrm{RB}$ and $\mathrm{RMB}$ refer to restricted kinetic and magnetic balance, while CGO and GIAO denote a common gauge origin and gauge-including atomic orbitals, respectively. We use the mSNSO-DLU-X2C Hamiltonian. The x2c-TZVPPall-2c/x2c-SVPall-2c basis sets are employed. We refer to the Supporting Information with the results obtained using the x2c-QZVPall-2c/x2c-SVPall-2c basis sets. Experimental (Expt.) results are taken from Ref. 8. Th experimental uncertainties are 0.002 .

\begin{tabular}{|c|c|c|c|c|c|c|c|c|c|c|}
\hline \multirow[b]{2}{*}{ Functional } & \multirow[b]{2}{*}{ Hamiltonian } & \multicolumn{3}{|c|}{$\left[\mathrm{La}\left(\mathrm{OAr}^{*}\right)_{3}\right]^{-}$} & \multicolumn{3}{|c|}{$\left[\mathrm{Lu}\left(\mathrm{NR}_{2}\right)_{3}\right]^{-}$} & \multicolumn{3}{|c|}{$\left[\mathrm{Lu}\left(\mathrm{OAr}^{*}\right)_{3}\right]^{-}$} \\
\hline & & $\mathrm{g}_{11}$ & $\mathrm{~g}_{22}$ & $\mathrm{~g}_{33}$ & $\mathrm{~g}_{11}$ & $\mathrm{~g}_{22}$ & $\mathrm{~g}_{33}$ & $\mathrm{~g}_{11}$ & $\mathrm{~g}_{22}$ & $\mathrm{~g}_{33}$ \\
\hline \multirow[t]{3}{*}{ TPSS } & RKB CGO & 1.897 & 1.897 & 1.998 & 1.885 & 1.885 & 1.995 & 1.907 & 1.907 & 1.995 \\
\hline & RMB CGO & 1.897 & 1.897 & 1.998 & 1.886 & 1.886 & 1.996 & 1.909 & 1.909 & 1.998 \\
\hline & RMB GIAO & 1.895 & 1.895 & 1.998 & 1.884 & 1.884 & 1.997 & 1.909 & 1.909 & 1.998 \\
\hline \multirow{3}{*}{$\mathrm{r}^{2} \mathrm{SCAN}$} & RKB CGO & 1.905 & 1.905 & 1.996 & 1.890 & 1.890 & 1.996 & 1.908 & 1.908 & 1.996 \\
\hline & RMB CGO & 1.907 & 1.908 & 1.998 & 1.891 & 1.891 & 1.998 & 1.911 & 1.911 & 1.998 \\
\hline & RMB GIAO & 1.902 & 1.903 & 1.998 & 1.887 & 1.887 & 1.998 & 1.908 & 1.908 & 1.999 \\
\hline \multirow[t]{3}{*}{ PBE0 } & RKB CGO & 1.886 & 1.886 & 1.995 & 1.886 & 1.886 & 1.996 & 1.906 & 1.906 & 1.996 \\
\hline & B CGO & 1.888 & 1.888 & 1.997 & 1.887 & 1.887 & 1.997 & .909 & 909 & 1.998 \\
\hline & RMB GIAO & 1.888 & 1.8 & 1.997 & 1.887 & 1.887 & 1.997 & 1.909 & 909 & 1.998 \\
\hline \multirow[t]{3}{*}{ PBE0-40HF } & RKB CGO & 1.893 & 1.894 & 1.997 & 1.891 & 1.891 & 1.996 & 1.912 & 1.917 & 1.997 \\
\hline & $\mathrm{GO}$ & 1.895 & 1.8 & 1.9 & 1.892 & 1.892 & 1.998 & 1.915 & 1.920 & 1.999 \\
\hline & RMB GIAO & 1.894 & 1.895 & 1.9 & 1.892 & 1.892 & 1.998 & 1.914 & 1.919 & 1.999 \\
\hline \multirow[t]{3}{*}{ CAM-QTP-02 } & RKB CGO & 1.890 & 1.892 & 1.997 & 1.878 & 1.878 & 1.996 & 1.900 & 1.901 & 1.996 \\
\hline & RMB CGO & 1.892 & 1.8 & 1.9 & 1.879 & 1.8 & 1.998 & 1.903 & 1.904 & 1.999 \\
\hline & RMB GIAO & 1.891 & 1.893 & 1.9 & 1.879 & 1.879 & 1.998 & 1.902 & 1.903 & 1.999 \\
\hline \multirow[t]{3}{*}{ HSE06 } & RKB CGO & 1.914 & 1.916 & 1.998 & 1.889 & 1.889 & 1.996 & 1.917 & 1.918 & 1.997 \\
\hline & RMB CGO & 1.916 & 1.918 & 1.999 & 1.890 & 1.890 & 1.998 & 1.919 & 1.921 & 1.999 \\
\hline & RMB GIAO & 1.913 & 1.915 & 1.999 & 1.890 & 1.890 & 1.998 & 1.917 & 1.919 & 1.999 \\
\hline \multirow{3}{*}{$\omega \mathrm{B} 97 \mathrm{X}-\mathrm{D}$} & RKB CGO & 1.879 & 1.880 & 1.996 & 1.875 & 1.877 & 1.996 & 1.900 & 1.901 & 1.996 \\
\hline & RMB CGO & 1.881 & 1.882 & 1.997 & 1.876 & 1.879 & 1.998 & 1.903 & 1.904 & 1.998 \\
\hline & RMB GIAO & 1.879 & 1.881 & 1.997 & 1.876 & 1.878 & 1.998 & 1.902 & 1.903 & 1.998 \\
\hline Expt. & & 1.876 & 1.886 & 2.000 & 1.882 & 1.898 & 2.000 & 1.915 & 1.915 & 2.000 \\
\hline
\end{tabular}

$\left[\mathrm{La}\left(\mathrm{OAr}^{*}\right)_{3}\right]^{-},\left[\mathrm{Lu}\left(\mathrm{NR}_{2}\right)_{3}\right]^{-}$, and $\left[\mathrm{Lu}\left(\mathrm{OAr}^{*}\right)_{3}\right]^{-}$using the RKB-CGO, RMB-CGO, and RMBGIAO approaches for a selection of density functionals evaluated in Section 6.3. Results obtained using the $\mathrm{x} 2 \mathrm{c}-\mathrm{TZVPP}$ all-2c/x2c-SVPall-2c basis set combination are provided in Table 6, whereas the results using the $\mathrm{x} 2 \mathrm{c}-\mathrm{QZVPall}-2 \mathrm{c} / \mathrm{x} 2 \mathrm{c}-\mathrm{SVPall}-2 \mathrm{c}$ basis sets are given as Supporting Information.

While in some cases the RMB GIAO ansatz yielded modest improvements to the predicted g-tensor components in $\left[\mathrm{La}\left(\mathrm{OAr}^{*}\right)_{3}\right]^{-},\left[\mathrm{Lu}\left(\mathrm{NR}_{2}\right)_{3}\right]^{-}$, and $\left[\mathrm{Lu}(\mathrm{OAr})_{3}\right]^{-}$, the overall differences 
between the three ansätze remained small, as observed in previous results reported above. In the following discussion, we therefore compare RMB GIAO results with experimental results, and errors in g-tensor components are computed as shifts in ppt by: $\Delta \mathrm{g}=\left(g-g_{\text {expt }}\right) \cdot 1000$.

$\left[\mathrm{La}\left(\mathrm{OAr}^{*}\right)_{3}\right]^{-}$appeared to be a particularly difficult case for the majority of the density functionals tested, with errors in $\mathrm{g}_{11}$ exceeding $15 \mathrm{ppt}$ for all functionals but $\omega \mathrm{B} 97 \mathrm{X}-\mathrm{D}$ and PBE0. In particular, HSE06 performed the worst for $\left[\mathrm{La}\left(\mathrm{OAr}^{*}\right)_{3}\right]^{-}$with errors in $\mathrm{g}_{11}$ and $\mathrm{g}_{22}$ of approximately $37 \mathrm{ppt}$ and $19 \mathrm{ppt}$, respectively, exceeding those of both $\mathrm{r}^{2} \mathrm{SCAN}$ and TPSS. The performance of all functionals were more consistent between themselves for complex $\left[\mathrm{Lu}\left(\mathrm{NR}_{2}\right)_{3}\right]^{-}$, with errors less than $20 \mathrm{ppt}$ for all principal components of the g-tensor. The $\mathrm{g}_{22}$ component gave rise to the largest errors, which were universally underestimated by all functionals but worst captured by $\omega$ B97X-D and CAM-QTP-02, giving 19 ppt error in each case. For compound $\left[\mathrm{Lu}\left(\mathrm{OAr}^{*}\right)_{3}\right]^{-}$, the $\omega \mathrm{B} 97 \mathrm{X}-\mathrm{D}$ and CAM-QTP-02 functionals again performed the worst for $\mathrm{g}_{11}$ and $\mathrm{g}_{22}$ with errors of over $12 \mathrm{ppt}$. In contrast, the hybrids and pure functionals performed well with errors of less than 8 ppt for both components. Surprisingly, HSE06 yielded the most accurate g-tensor for this compound, despite the fact that it performed the worst for $\left[\mathrm{La}\left(\mathrm{OAr}^{*}\right)_{3}\right]^{-}$. These observations also hold for predictions of the g-tensor obtained using the x2c-QZVPall-2c/x2c-SVPall-2c basis sets, which is included in the Supporting Information. This confirms the findings of Sec. 6.2 and shows that triple- $\zeta$ basis sets are sufficient for the calculation of the g-tensor.

The results indicate that accurate g-tensors for the three Ln compounds can be obtained using local exact two-component theory. Errors in the principal components of the g-tensor do not exceed $40 \mathrm{ppt}$ for all functionals tested. However, due to the inconsistent accuracy of the range-separated hybrids between $\left[\mathrm{La}\left(\mathrm{OAr}^{*}\right)_{3}\right]^{-},\left[\mathrm{Lu}\left(\mathrm{NR}_{2}\right)_{3}\right]^{-}$, and $\left[\mathrm{Lu}(\mathrm{OAr})_{3}\right]^{-}$, hybrid functionals may be better suited for general application to these types of complexes. 


\section{Conclusions}

A gauge-origin invariant formulation of exact two-component (X2C) theory for EPR g-tensors has been presented. This includes the complete derivative of the X2C Hamiltonian in the presence of an external magnetic field. Gauge-including atomic orbitals (GIAOs) and the restricted magnetic balance $(\mathrm{RMB})$ condition are applied for the basis set expansion of the Dirac-Hamilton operator. Our implementation supports the finite nucleus model throughout, is fully integral direct, and parallelized using OpenMP. Furthermore, we apply the diagonal local approximation to the unitary decoupling transformation (DLU) to further reduce the computational costs. Together with our previously presented implementation of the EPR hyperfine coupling (HFC) constant, $\frac{73}{73}$ routine calculations are now possible for large molecules with several thousand basis functions.

Explicit comparisons between the four-component Dirac-Kohn-Sham (DKS) approach and $\mathrm{X} 2 \mathrm{C} / \mathrm{DLU}-\mathrm{X} 2 \mathrm{C}$ show that the two-component ansatz accurately reproduces the results of the "fully" relativistic theory when accounting for the two-electron picture-change error with the modified screened nuclear spin-orbit (mSNSO) approximation. The error introduced by DLU is negligible and considerably smaller than the error introduced by neglecting the decoupling derivatives as done in previous implementations. ${ }^{77}$ We assess the importance of GIAOs and the RMB condition for transition-metal complexes and lanthanide compounds. Here, the impact is found to be rather small for complexes consisting of one metal center, where the position of the common gauge origin is straightforward. The computational effort is dominated by the self-consistent field ( $\mathrm{SCF}$ ) procedure. Therefore, the gauge-invariant ansatz does not notably increase the computational costs as demonstrated for $\left[\mathrm{Pt}\left(\mathrm{C}_{6} \mathrm{Cl}_{5}\right)_{4}\right]^{-}$ with more than 3000 basis functions.

In addition, we carry out extensive benchmark studies with 24 basis sets and 22 density functional approximations. This complements our previous study for the HFC constant ${ }^{73}$ and reveals some notable differences for a computational treatment of the EPR g-tensor and HFC constant. For the g-tensor, triple- $\zeta$ basis sets such as the Sapporo, Dyall, or the Karlsruhe 
x2c-type bases are generally sufficient, whereas the HFC constant requires larger basis sets or even fully decontracted bases. Therefore, we recommend the uncontracted Dyall-VTZ or segmented-contracted x2c-QZVPall-2c basis set for an error-balanced description of the two EPR parameters. This is especially important for relativistic calculations of the pseudocontact shift for paramagnetic NMR spectra as this term consists of both the g-tensor and the HFC tensor.

Hybrid density functional approximations are required for accurate results. Compared to the HFC constant, a smaller amount of HF exchange is advantageous as evident from the performance of PBE0 and its modification with $40 \%$ of Harteee-Fock (HF) exchange. TPSSh and revTPSSh (10\% HF exchange) are not sufficient, whereas TPSS0 (25\% HF exchange) delivers good results. Thus, functionals with around $25 \%$ of HF exchange are a reasonable choice for the g-tensor. Range-separated hybrids are not a consistent improvement over conventional global hybrid functionals. CAM-B3LYP and $\omega$ B97-X perform best for the set of 17 transition-metal complexes, whereas CAM-QTP-00 leads to comparably large errors. The performance of the latter significantly depends on the molecular system, e.g., CAMQTP-00 is among the top performers for the larger complex $\left[\mathrm{Pt}\left(\mathrm{C}_{6} \mathrm{Cl}_{5}\right)_{4}\right]^{-}$. Overall, PBE0, LC- $\omega \mathrm{PBE}$, and $\omega \mathrm{B} 97-\mathrm{X}$ yield similar errors for the g-tensor and the HFC tensor.

Finally, the applicability of our implementation is demonstrated for the three spin- $(1 / 2)$ lanthanide compounds $\left[\mathrm{La}\left(\mathrm{OAr}^{*}\right)_{3}\right]^{-},\left[\mathrm{Lu}\left(\mathrm{NR}_{2}\right)_{3}\right]^{-}$, and $\left[\mathrm{Lu}\left(\mathrm{OAr}^{*}\right)_{3}\right]^{-}$, whose magnetic properties suggest design principles for engineering qubits using molecular spins. The principal components of the g-tensor reproduce experimental results well using x2c-TZVPPall-2c basis sets for the Ln atom and x2c-SVPall-2c basis sets for the ligand atoms. Unlike for the hyperfine coupling constant, the accuracy of range-separated hybrid functionals was inconsistent between the three Ln complexes, and thus hybrid functionals appear to provide more balanced accuracy for Ln species. 


\section{A Appendix}

\section{A.1 Simplification with Common Gauge Origin}

Without GIAOs, no derivatives of the two-electron integrals will arise and the g-tensor reduces to

$$
\frac{\mathrm{d} E}{\mathrm{~d} B_{u}}=\operatorname{tr}\left[\boldsymbol{P}\left(\overrightarrow{J_{v}}\right) \boldsymbol{h}_{u}^{B}\right]
$$

Note that we use the generalized kinetic energy density ${ }^{152}$ for meta-generalized gradient approximations only with the GIAO-based approach. Thus, only the derivative of the oneelectron Hamiltonian is required and this derivative is directly contracted with the SCF density matrix as done for the hyperfine coupling tensor. $\frac{73 / 75}{77}$ Neglecting GIAOs in the relativistic $4 \mathrm{c}$ basis set expansion leads to ${ }^{79}$

$$
\begin{aligned}
\left|\psi_{i}^{L}\right\rangle & =\sum_{\mu} c_{\mu i}^{L}\left|\phi_{\mu}\right\rangle \\
\left|\psi_{i}^{S}\right\rangle & =\sum_{\mu} c_{\mu i}^{S} \frac{1}{2 c} \vec{\sigma} \cdot\left(\hat{\vec{p}}+\frac{1}{c} \hat{\overrightarrow{\mathcal{A}}}\right)\left|\phi_{\mu}\right\rangle
\end{aligned}
$$

where $O$ refers to the common gauge origin (CGO), which is usually placed at the center of the heaviest atom or the center of mass. Then, the overlap matrix $\boldsymbol{S}$ and the potential matrix $\boldsymbol{V}$ are given by

$$
\begin{aligned}
& \boldsymbol{S}=\left(\begin{array}{ll}
\mathbf{S} & 0 \\
0 & \mathbf{S}
\end{array}\right), \mathbf{S}_{\mu \nu}=\left\langle\lambda_{\mu} \mid \lambda_{\nu}\right\rangle \\
& \boldsymbol{V}=\left(\begin{array}{cc}
\mathbf{V} & 0 \\
0 & \mathbf{V}
\end{array}\right), \mathbf{V}_{\mu \nu}=\left\langle\lambda_{\mu}|\hat{V}| \lambda_{\nu}\right\rangle
\end{aligned}
$$

All other matrices are still not block-diagonal and read

$$
\boldsymbol{\Pi}_{\mu \nu}^{\dagger}=\frac{1}{2}\left\langle\phi_{\mu}\left|\left[\vec{\sigma} \cdot\left(\hat{\vec{p}}+\frac{1}{c} \hat{\overrightarrow{\mathcal{A}}}_{O}\right)\right]\left[\vec{\sigma} \cdot\left(\hat{\vec{p}}+\frac{1}{c} \hat{\overrightarrow{\mathcal{A}}}_{O}\right)\right]\right| \phi_{\nu}\right\rangle
$$




$$
\begin{aligned}
\boldsymbol{\Pi}_{\mu \nu} & =\frac{1}{2}\left\langle\phi_{\mu}\left|\left[\vec{\sigma} \cdot\left(\hat{\vec{p}}+\frac{1}{c} \hat{\overrightarrow{\mathcal{A}}}_{O}\right)\right]\left[\vec{\sigma} \cdot\left(\hat{\vec{p}}+\frac{1}{c} \hat{\overrightarrow{\mathcal{A}}}_{O}\right)\right]\right| \phi_{\nu}\right\rangle \\
\boldsymbol{W}_{\mu \nu} & =\left\langle\phi_{\mu}\left|\left[\vec{\sigma} \cdot\left(\hat{\vec{p}}+\frac{1}{c} \hat{\overrightarrow{\mathcal{A}}}_{O}\right)\right] \hat{V}\left[\vec{\sigma} \cdot\left(\hat{\vec{p}}+\frac{1}{c} \hat{\overrightarrow{\mathcal{A}}}_{O}\right)\right]\right| \phi_{\nu}\right\rangle, \\
\boldsymbol{T}_{\mu \nu} & =\frac{1}{2}\left\langle\phi_{\mu}\left|\left[\vec{\sigma} \cdot\left(\hat{\vec{p}}+\frac{1}{c} \hat{\overrightarrow{\mathcal{A}}}_{O}\right)\right]\left[\vec{\sigma} \cdot\left(\hat{\vec{p}}+\frac{1}{c} \hat{\overrightarrow{\mathcal{A}}}_{O}\right)\right]\right| \phi_{\nu}\right\rangle
\end{aligned}
$$

All matrices are Hermitian. Note that the derivatives of the overlap and the potential matrix with respect to the magnetic field will be zero. So, the response equations of Sec. 2.4 are simplified. The derivatives of $\boldsymbol{T}, \boldsymbol{\Pi}$, and $\boldsymbol{W}$ are still non-zero and given by

$$
\begin{aligned}
\left(\frac{\partial \boldsymbol{T}_{\mu \nu}}{\partial B_{u}}\right)_{0}= & \frac{1}{2 c} \sigma_{0}\left\langle\lambda_{\mu}\left|\left(\hat{\vec{r}}_{O} \times \hat{\vec{p}}\right)_{u}\right| \lambda_{\nu}\right\rangle+\frac{1}{2 c} \sigma_{u}\left\langle\lambda_{\mu} \mid \lambda_{\nu}\right\rangle \\
\left(\frac{\partial \boldsymbol{\Pi}_{\mu \nu}^{\dagger}}{\partial B_{u}}\right)_{0}= & \frac{1}{2 c} \sigma_{0}\left\langle\lambda_{\mu}\left|\left(\hat{\vec{r}}_{O} \times \hat{\vec{p}}\right)_{u}\right| \lambda_{\nu}\right\rangle+\frac{1}{2 c} \sigma_{u}\left\langle\lambda_{\mu} \mid \lambda_{\nu}\right\rangle \\
\left(\frac{\partial \boldsymbol{W}_{\mu \nu}}{\partial B_{u}}\right)_{0}= & +\frac{1}{2 c} \sigma_{0}\left\langle\lambda_{\mu}\left|\left(\hat{\vec{r}}_{O} \times \hat{\vec{p}}\right)_{u} \hat{V}+\hat{V}\left(\hat{\vec{r}}{ }_{O} \times \hat{\vec{p}}\right)_{u}\right| \lambda_{\nu}\right\rangle \\
& +\left\langle\lambda_{\mu}\left|\frac{\mathrm{i}}{2 c} \sigma_{u}(\hat{\vec{p}} \hat{V}) \cdot \hat{\vec{r}}_{O}-\frac{\mathrm{i}}{2 c}(\hat{\vec{p}} \hat{V})_{u}\left(\vec{\sigma} \cdot \hat{\vec{r}}_{O}\right)\right| \lambda_{\nu}\right\rangle \\
& +\frac{1}{c} \sigma_{u}\left\langle\lambda_{\mu}|\hat{V}| \lambda_{\nu}\right\rangle
\end{aligned}
$$

Note that for the position vector $\vec{r}$ the subscript $\mu$ is replaced by the common gauge origin O compared to Sec. 2.4. The derivative of the X2C Hamiltonian becomes

$$
\boldsymbol{h}_{u}^{+, B}=\boldsymbol{R}_{u}^{\dagger, B} \boldsymbol{L} \boldsymbol{R}+\boldsymbol{R}^{\dagger} \boldsymbol{L}_{u}^{B} \boldsymbol{R}+\boldsymbol{R}^{\dagger} \boldsymbol{L} \boldsymbol{R}_{u}^{B}
$$

where the derivative of the NESC matrix reads

$$
\begin{aligned}
\boldsymbol{L}_{u}^{B}= & \boldsymbol{\Pi}_{u}^{\dagger, B} \boldsymbol{X}+\boldsymbol{\Pi}^{\dagger} \boldsymbol{X}_{u}^{B}+\boldsymbol{X}_{u}^{\dagger, B} \boldsymbol{\Pi}+\boldsymbol{X}^{\dagger} \boldsymbol{\Pi}_{u}^{B} \\
+ & \boldsymbol{X}_{u}^{\dagger, B}\left(\frac{1}{4 c^{2}} \boldsymbol{W}-\boldsymbol{T}\right) \mathbf{X}+\boldsymbol{X}^{\dagger}\left(\frac{1}{4 c^{2}} \boldsymbol{W}_{u}^{B}-\boldsymbol{T}_{u}^{B}\right) \boldsymbol{X}+\boldsymbol{X}^{\dagger}\left(\frac{1}{4 c^{2}} \boldsymbol{W}-\boldsymbol{T}\right) \boldsymbol{X}_{u}^{B}
\end{aligned}
$$

Compared to the GIAO-based equation, only the derivative of the potential matrix $\boldsymbol{V}$ is omitted. 
As the derivative of $\boldsymbol{T}$ is still non-zero, the derivative of the $4 \mathrm{c}$ metric $\mathbb{M}$ is also non-zero in RMB. Thus, the working equations for the derivative of $\boldsymbol{X}$ are

$$
\boldsymbol{X}_{u}^{B}=\left(\boldsymbol{C}_{-}^{S}-\boldsymbol{X} \mathbf{C}_{-}^{L}\right)\left(\boldsymbol{U}_{u}^{B}\right)_{-+} \boldsymbol{C}_{+}^{L, \dagger} \tilde{\boldsymbol{S}}
$$

with the orbital rotation matrix

$$
\left(\boldsymbol{U}_{u,-+}^{B}\right)_{k l}=\frac{\left(\tilde{\boldsymbol{D}}_{u,-+}^{B}\right)_{k l}-\left(\tilde{\boldsymbol{M}}_{u,-+}^{B}\right)_{k l}\left(\boldsymbol{E}_{++}\right)_{l l}}{\left(\boldsymbol{E}_{++}\right)_{l l}-\left(\boldsymbol{E}_{--}\right)_{k k}}
$$

The derivatives of the Dirac matrix and the metric of the Dirac equation are transformed to the basis of the unperturbed solutions according to

$$
\begin{aligned}
\tilde{\boldsymbol{D}}_{u,-+}^{B}= & \boldsymbol{C}_{-}^{L, \dagger} \boldsymbol{\Pi}_{u}^{\dagger, B} \boldsymbol{C}_{+}^{S}+\boldsymbol{C}_{-}^{S, \dagger} \boldsymbol{\Pi}_{u}^{B} \boldsymbol{C}_{+}^{L} \\
& +\frac{1}{4 c^{2}} \boldsymbol{C}_{-}^{S, \dagger} \boldsymbol{W}_{u}^{B} \boldsymbol{C}_{+}^{S}-\boldsymbol{C}_{-}^{S, \dagger} \boldsymbol{T}_{u}^{B} \boldsymbol{C}_{+}^{S} \\
\tilde{\boldsymbol{M}}_{u,-+}^{B}= & \frac{1}{2 c^{2}} \boldsymbol{C}_{-}^{S, \dagger} \boldsymbol{T}_{u}^{B} \boldsymbol{C}_{+}^{S}
\end{aligned}
$$

Furthermore, the derivatives of the renormalization matrix are calculated by solving a simplified version of the Sylvester matrix equation according to

$$
\boldsymbol{R} \boldsymbol{R}_{u}^{B}+\boldsymbol{R}_{u}^{B} \boldsymbol{R}=-\tilde{\boldsymbol{S}}^{-1} \tilde{\boldsymbol{S}}_{u}^{B} \boldsymbol{R} \boldsymbol{R}
$$

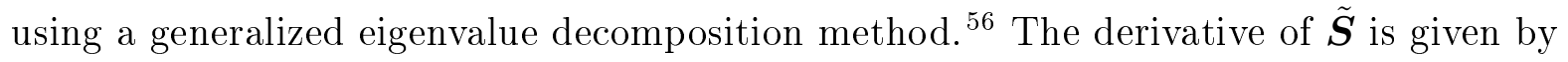

$$
\tilde{\boldsymbol{S}}_{u}^{B}=\frac{1}{2 c^{2}} \boldsymbol{X}_{u}^{\dagger, B} \boldsymbol{T} \boldsymbol{X}+\frac{1}{2 c^{2}} \boldsymbol{X}^{\dagger} \boldsymbol{T}_{u}^{B} \boldsymbol{X}+\frac{1}{2 c^{2}} \boldsymbol{X}^{\dagger} \boldsymbol{T} \boldsymbol{X}_{u}^{B}
$$




\section{A.2 Simplification with Kinetic Balance}

Further approximations are possible, i.e. the generalized momentum operator can be neglected like in $4 \mathrm{c}$ approaches. ${ }^{28129}$ Thus, the basis set expansion reads ${ }^{78}$

$$
\begin{aligned}
\left|\psi_{i}^{L}\right\rangle & =\sum_{\mu} c_{\mu i}^{L}\left|\phi_{\mu}\right\rangle \\
\left|\psi_{i}^{S}\right\rangle & =\sum_{\mu} c_{\mu i}^{S} \frac{1}{2 c} \vec{\sigma} \cdot \hat{\vec{p}}\left|\phi_{\mu}\right\rangle
\end{aligned}
$$

The Dirac equation in the supermatrix form consists of the block-diagonal matrices

$$
\begin{aligned}
& \boldsymbol{S}=\left(\begin{array}{ll}
\mathbf{S} & 0 \\
0 & \mathrm{~S}
\end{array}\right), \mathbf{S}_{\mu \nu}=\left\langle\lambda_{\mu} \mid \lambda_{\nu}\right\rangle \\
& \boldsymbol{V}=\left(\begin{array}{ll}
\mathbf{V} & 0 \\
0 & \mathbf{V}
\end{array}\right), \mathbf{V}_{\mu \nu}=\left\langle\lambda_{\mu}|\hat{V}| \lambda_{\nu}\right\rangle \\
& \boldsymbol{T}=\left(\begin{array}{ll}
\mathbf{T} & 0 \\
0 & \mathbf{T}
\end{array}\right), \mathbf{T}_{\mu \nu}=\frac{1}{2}\left\langle\phi_{\mu}\left|\hat{p}^{2}\right| \phi_{\nu}\right\rangle
\end{aligned}
$$

and the general matrices

$$
\begin{aligned}
\boldsymbol{\Pi}_{\mu \nu}^{\dagger} & =\frac{1}{2}\left\langle\phi_{\mu}\left|\left[\vec{\sigma} \cdot\left(\hat{\vec{p}}+\frac{1}{c} \hat{\overrightarrow{\mathcal{A}}}_{O}\right)\right](\vec{\sigma} \cdot \hat{\vec{p}})\right| \phi_{\nu}\right\rangle \\
\boldsymbol{W}_{\mu \nu} & =\left\langle\phi_{\mu}|(\vec{\sigma} \cdot \hat{\vec{p}}) \hat{V}(\vec{\sigma} \cdot \hat{\vec{p}})| \phi_{\nu}\right\rangle
\end{aligned}
$$

Only the generalized momentum matrix $\boldsymbol{\Pi}$ depends on the vector potential. Therefore, the derivative of the $\mathrm{X} 2 \mathrm{C}$ Hamiltonian is considerably simplified to

$$
\boldsymbol{h}_{u}^{+, B}=\boldsymbol{R}_{u}^{\dagger, B} \boldsymbol{L} \boldsymbol{R}+\boldsymbol{R}^{\dagger} \boldsymbol{L}_{u}^{B} \boldsymbol{R}+\boldsymbol{R}^{\dagger} \boldsymbol{L} \boldsymbol{R}_{u}^{B}
$$


where the derivative of the NESC matrix reads

$$
\begin{aligned}
\boldsymbol{L}_{u}^{B}= & \boldsymbol{\Pi}_{u}^{\dagger, B} \boldsymbol{X}+\boldsymbol{\Pi}^{\dagger} \boldsymbol{X}_{u}^{B}+\boldsymbol{X}_{u}^{\dagger, B} \boldsymbol{\Pi}+\boldsymbol{X}^{\dagger} \boldsymbol{\Pi}_{u}^{B} \\
+ & \boldsymbol{X}_{u}^{\dagger, B}\left(\frac{1}{4 c^{2}} \boldsymbol{W}-\boldsymbol{T}\right) \mathbf{X}+\boldsymbol{X}^{\dagger}\left(\frac{1}{4 c^{2}} \boldsymbol{W}-\boldsymbol{T}\right) \boldsymbol{X}_{u}^{B}
\end{aligned}
$$

Here, the one-electron integral derivative of the generalized momentum matrix is given by

$$
\begin{aligned}
\left(\frac{\partial \boldsymbol{\Pi}_{\mu \nu}^{\dagger}}{\partial B_{u}}\right)_{0} & =\frac{1}{4 c}\left\langle\phi_{\mu}\left|\left(\hat{\vec{r}}_{O} \times \vec{\sigma}\right)_{u} \vec{\sigma} \cdot \hat{\vec{p}}\right| \phi_{\nu}\right\rangle \\
& =\frac{1}{4 c} \sigma_{0}\left\langle\lambda_{\mu}\left|\left(\hat{\vec{r}}_{O} \times \hat{\vec{p}}\right)_{u}\right| \lambda_{\nu}\right\rangle+\frac{\mathrm{i}}{4 c}\left\langle\lambda_{\mu}\left|\left[\vec{\sigma} \cdot \hat{\vec{r}}_{O}\right] \hat{p}_{u}-\sigma_{u}\left[\hat{\vec{r}}_{O} \cdot \hat{\vec{p}}\right]\right| \lambda_{\nu}\right\rangle
\end{aligned}
$$

Thus, only the prefactor of the scalar part is changed compared to the RMB integral of this derivative. The explicit working equations for the decoupling derivatives read

$$
\boldsymbol{X}_{u}^{B}=\left(\boldsymbol{C}_{-}^{S}-\boldsymbol{X} \boldsymbol{C}_{-}^{L}\right)\left(\boldsymbol{U}_{u}^{B}\right)_{-+} \boldsymbol{C}_{+}^{L, \dagger} \tilde{\boldsymbol{S}}
$$

with the orbital rotation matrix

$$
\left(\boldsymbol{U}_{u,-+}^{B}\right)_{k l}=\frac{\left(\tilde{\boldsymbol{D}}_{u,-+}^{B}\right)_{k l}}{\left(\boldsymbol{E}_{++}\right)_{l l}-\left(\boldsymbol{E}_{--}\right)_{k k}}
$$

as the derivatives of the metric vanish. The derivatives of the Dirac matrix are transformed to the basis of the unperturbed solutions according to

$$
\tilde{\boldsymbol{D}}_{u,-+}^{B}=\boldsymbol{C}_{-}^{L, \dagger} \boldsymbol{\Pi}_{u}^{\dagger, B} \boldsymbol{C}_{+}^{S}+\boldsymbol{C}_{-}^{S, \dagger} \boldsymbol{\Pi}_{u}^{B} \boldsymbol{C}_{+}^{L}
$$

Furthermore, the derivatives of the renormalization matrix are calculated by solving a simplified version of the Sylvester matrix equation according to

$$
\boldsymbol{R} \boldsymbol{R}_{u}^{B}+\boldsymbol{R}_{u}^{B} \boldsymbol{R}=-\tilde{\boldsymbol{S}}^{-1} \tilde{\boldsymbol{S}}_{u}^{B} \boldsymbol{R} \boldsymbol{R}
$$


using a generalized eigenvalue decomposition method. $\frac{56}{}$ The derivative of $\tilde{\boldsymbol{S}}$ is given by

$$
\tilde{\boldsymbol{S}}_{u}^{B}=\frac{1}{2 c^{2}} \boldsymbol{X}_{u}^{\dagger, B} \boldsymbol{T} \boldsymbol{X}+\frac{1}{2 c^{2}} \boldsymbol{X}^{\dagger} \boldsymbol{T} \boldsymbol{X}_{u}^{B}
$$

Thus, the response and Sylvester matrix equations feature the same structural form as for the hyperfine coupling tensor. ${ }^{73}$

\section{A.3 Pseudo-Contact Shift for paramagnetic NMR}

The evaluation of the EPR hyperfine coupling matrix and the g-matrix further extends to the hyperfine contribution to the paramagnetic NMR (pNMR) shielding tensor $\boldsymbol{\sigma}_{I}$ of nucleus $I$ according to 236

$$
\boldsymbol{\sigma}_{I}^{\mathrm{tot}}=\boldsymbol{\sigma}_{I}^{\mathrm{orb}}-\frac{\mu_{e} \tilde{S}(\tilde{S}+1)}{3 \gamma_{I} k_{B} T} \boldsymbol{A}_{I} \boldsymbol{g}^{\mathrm{T}}
$$

where $\mu_{e}$ denotes the Bohr magneton, $\gamma_{I}$ the gyromagnetic ratio, $k_{B}$ the Boltzmann constant, and $T$ the temperature. $\tilde{S}$ is the expectation value of the effective spin. Note that we use the canonical form of the shielding tensor. ${ }^{237 / 238}$ Here, we neglected zero-field splitting. Composing the HFC and the g-tensor into its isotropic and anisotropic contribution is done via

$$
\begin{aligned}
\boldsymbol{M} & =M^{\text {iso }}+\boldsymbol{M}^{\text {aniso }} \\
M^{\text {iso }} & =\frac{1}{3} \operatorname{tr}(\boldsymbol{M}) \\
\boldsymbol{M}^{\text {aniso }} & =\boldsymbol{M}-M^{\text {iso }} \mathbf{1}
\end{aligned}
$$

Note that this partitioning is necessary due to spin-orbit coupling as discussed in Ref. 236. Therefore, the two-component form of the Fermi-contact (FC) and the pseudo-contact (PC) contribution to the pNMR shielding constant reads

$$
\sigma_{I}^{\mathrm{FC}}=\frac{\mu_{e} \tilde{S}(\tilde{S}+1)}{3 \gamma_{I} k_{B} T} A^{\text {iso }} g^{\text {iso }}
$$




$$
\sigma_{I}^{\mathrm{PC}}=\frac{\mu_{e} \tilde{S}(\tilde{S}+1)}{3 \gamma_{I} k_{B} T} \operatorname{tr}\left(\boldsymbol{A}^{\text {aniso }} \boldsymbol{g}^{\text {aniso, } \mathrm{T}}\right)
$$

The $\mathrm{FC}$ and the $\mathrm{PC}$ term for $\mathrm{pNMR}$ can be readily evaluated with the presented $\mathrm{X} 2 \mathrm{C}$ implementation as demonstrated in the Supporting Information.

\section{Acknowledgement}

Y.J.F. acknowledges financial support by TURBOMOLE GmbH and was further supported by a fellowship of Fonds der Chemischen Industrie (FCI, German Chemical Industry Funds). J.M.Y. acknowledges support from the National Science Foundation under grant No. DCE1839285. A portion of the calculations reported in this work was performed using the highperformance computing (HPC) infrastructure available at the Research Cyberinfrastructure Center (RCIC) at the University of California, Irvine (UCI) and supported by the National Science Foundation under grant No. CNS-1828779. Another portion of the calculations were performed using hardware funded by Fonds der Chemischen Industrie. We are grateful to Filipp Furche and Florian Weigend for fruitful discussions.

\section{Supporting Information Available}

Supporting Information is available

- Further studies on the relativistic Hamiltonian, the basis set, density functional approximation, and the impact of the structure for the set of diatomic molecules in Sec. 5 $(\mathrm{PDF})$

- Comparison of the SNSO and mSNSO approximation to the one-electron X2C ansatz without corrections for Sec. 6.1 (PDF)

- Results and assessment of DLU error for the Sapporo basis sets featuring diffuse functions for Sec. 6.1 (PDF) 
- Individual results for the basis set study in Sec. 6.2 (PDF)

- Individual results for the DFT study in Sec. 6.3 (PDF)

- Results for the lanthanide compounds of Sec. 7 with the x2c-QZVPall-2c basis set $(\mathrm{PDF})$

- Application of the g-tensor and HFC implementation to calculate the temperaturedependent terms for pNMR shielding constants as outlined in Sec. A.3 (PDF)

- Uncontracted x2c-type basis sets for Sec.6.2, i.e. x2c-SVPall-2c-s and x2c-TZVPall-2c-s (txt)

\section{References}

(1) Kaupp, M., Bühl, M., Malkin, V. G., Eds. Calculation of NMR and EPR Parameters: Theory and Applications; Wiley-VCH: Weinheim, Germany, 2004.

(2) Neese, F. Quantum Chemistry and EPR Parameters. In eMagRes; American Cancer Society, 2017; Vol. 6; pp 1-22.

(3) McWeeny, R. On the Origin of Spin-Hamiltonian Parameters. J. Phys. Chem. 1965, 42, 1717-1725.

(4) McWeeny, R. Spins in Quantum Chemistry; Academic Press: New York, USA, 1970.

(5) Harriman, J. E. Theoretical Foundations of Electron Spin Resonance; Physical Chemistry: A Series of Monographs; Academic Press: New York, USA, 1978; Vol. 37.

(6) McWeeny, R. Methods of molecular quantum mechanics, 2nd ed.; Academic Press: London, United Kingdom, 1989.

(7) Goldfarb, D., Stoll, S., Eds. EPR Spectroscopy: Fundamentals and Methods; John Wiley \& Sons: Chichester, United Kingdom, 2018. 
(8) Kundu, K.; White, J. R. K.; Moehring, S. A.; Yu, J. M.; Ziller, J. W.; Furche, F.; Evans, W. J.; Hill, S. Clock Transition Due to a Record 1240 G Hyperfine Interaction in a Lu(II) Molecular Spin Qubit. ChemRxiv 2021, DOI: 10.26434/chemrxiv.14399333.v1.

(9) Briganti, M.; Lucaccini, E.; Chelazzi, L.; Ciattini, S.; Sorace, L.; Sessoli, R.; Totti, F.; Perfetti, M. Magnetic Ansiotropy Trends along a Full 4f-Series: The $\mathrm{f}^{n+7}$ Effect. J. Am. Chem. Soc. 2021, 143, 8108-815.

(10) Pyykkö, P. Relativistic Effects in Chemistry: More Common Than You Thought. Annu. Rev. Phys. Chem. 2012, 63, 45-64.

(11) Saue, T. Relativistic Hamiltonians for Chemistry: A Primer. ChemPhysChem 2011, 12, 3077-3094.

(12) Dyall, K. G.; Fægri Jr., K. Introduction to Relativistic Quantum Chemistry; Oxford University Press: New York, USA, 2007.

(13) Reiher, M.; Wolf., A. Relativistic Quantum Chemistry - The Fundamental Theory of Molecular Science, 2nd ed.; Wiley-VCH: Weinheim, Germany, 2015.

(14) Liu, W. Ideas of relativistic quantum chemistry. Mol. Phys. 2010, 108, 1679-1706.

(15) Liu, W., Ed. Handbook of Relativistic Quantum Chemistry; Springer: Berlin, Heidelberg, Germany, 2017.

(16) Liu, W. Essentials of relativistic quantum chemistry. J. Chem. Phys. 2020, 152, 180901.

(17) Autschbach, J. Relativistic Effects on Magnetic Resonance Parameters and Other Properties of Inorganic Molecules and Metal Complexes. In Relativistic Methods for Chemists; Barysz, M., Ishikawa, Y., Eds.; Springer Netherlands: Dordrecht, The Netherlands, 2010; pp 521-598. 
(18) Autschbach, J. Perspective: Relativistic effects. J. Chem. Phys. 2012, 136, 150902.

(19) Autschbach, J. Relativistic calculations of magnetic resonance parameters: background and some recent developments. Phil. Trans. R. Soc. A. 2014, 372, 20120489.

(20) Chang, C.; Pelissier, M.; Durand, P. Regular Two-Component Pauli-Like Effective Hamiltonians in Dirac Theory. Phys. Scr. 1986, 34, 394-404.

(21) van Lenthe, E.; Baerends, E. J.; Snijders, J. G. Relativistic regular two-component Hamiltonians. J. Phys. Chem. 1993, 99, 4597-4610.

(22) van Lenthe, E.; Baerends, E. J.; Snijders, J. G. Relativistic total energy using regular approximations. J. Chem. Phys. 1994, 101, 9783-9792.

(23) van Lenthe, E.; Wormer, P. E. S.; van der Avoird, A. Density functional calculations of molecular g-tensors in the zero-order regular approximation for relativistic effects. J. Chem. Phys. 1997, 107, 2488-2498.

(24) Autschbach, J.; Pritchard, B. Calculation of molecular g-tensors using the zeroth-order regular approximation and density functional theory: expectation value versus linear response approaches. Theor. Chem. Acc. 2011, 129, 453-466.

(25) Aquino, F.; Govind, N.; Autschbach, J. Scalar Relativistic Computations of Nuclear Magnetic Shielding and g-Shifts with the Zeroth-Order Regular Approximation and Range-Separated Hybrid Density Functionals. J. Chem. Theory Comput. 2011, 7, $3278-3292$.

(26) Verma, P.; Autschbach, J. Variational versus Perturbational Treatment of Spin-Orbit Coupling in Relativistic Density Functional Calculations of Electronic g Factors: Effects from Spin-Polarization and Exact Exchange. J. Chem. Theory Comput. 2013, 9, 1052-1067. 
(27) Malkin, I.; Malkina, O. L.; Malkin, V. G.; Kaupp, M. Relativistic two-component calculations of electronic g-tensors that include spin polarization. J. Chem. Phys. 2005, 123, 244103.

(28) Repiský, M.; Komorovský, S.; Malkin, E.; Malkina, O. L.; Malkin, V. G. Relativistic four-component calculations of electronic g-tensors in the matrix Dirac-Kohn-Sham framework. Chem. Phys. Lett. 2010, 488, 94-97.

(29) Gohr, S.; Hrobárik, P.; Repiský, M.; Komorovský, S.; Ruud, K.; Kaupp, M. FourComponent Relativistic Density Functional Theory Calculations of EPR g- and Hyperfine-Coupling Tensors Using Hybrid Functionals: Validation on TransitionMetal Complexes with Large Tensor Anisotropies and Higher-Order Spin-Orbit Effects. J. Phys. Chem. A 2015, 119, 12892-12905.

(30) Cherry, P. J.; Komorovsky, S.; Malkin, V. G.; Malkina, O. L. Calculations of the EPR g-tensor using unrestricted two- and four-component relativistic approaches within the HF and DFT frameworks. Mol. Phys. 2017, 115, 75-89.

(31) Remigio, R. D.; Repisky, M.; Komorovsky, S.; Hrobarik, P.; Frediani, L.; Ruud, K. Four-component relativistic density functional theory with the polarisable continuum model: application to EPR parameters and paramagnetic NMR shifts. Mol. Phys. 2017, 115, 214-227.

(32) Kutzelnigg, W.; Liu, W. Quasirelativistic theory equivalent to fully relativistic theory. J. Chem. Phys. 2005, 123, 241102.

(33) Liu, W.; Kutzelnigg, W. Quasirelativistic theory. II. Theory at matrix level. J. Chem. Phys. 2007, 126, 114107.

(34) Liu, W.; Peng, D. Infinite-order quasirelativistic density functional method based on the exact matrix quasirelativistic theory. J. Chem. Phys. 2006, 125, 044102. 
(35) Liu, W.; Peng, D. Erratum: "Infinite-order quasirelativistic density functional method based on the exact matrix quasirelativistic theory" [J. Chem. Phys. 125, 044102 (2006)]. J. Chem. Phys. 2006, 125, 149901.

(36) Iliaš, M.; Saue, T. An infinite-order two-component relativistic Hamiltonian by a simple one-step transformation. J. Chem. Phys. 2007, 126, 064102.

(37) Liu, W.; Peng, D. Exact two-component Hamiltonians revisited. J. Chem. Phys. 2009, $131,031104$.

(38) Peng, D.; Liu, W.; Xiao, Y.; Cheng, L. Making four- and two-component relativistic density functional methods fully equivalent based on the idea of "from atoms to molecule". J. Chem. Phys. 2007, 12\%, 104106.

(39) Repisky, M.; Komorovsky, S.; Kadek, M.; Konecny, L.; Ekström, U.; Malkin, E.; Kaupp, M.; Ruud, K.; Malkina, O. L.; Malkin, V. G. ReSpect: Relativistic spectroscopy DFT program package. J. Chem. Phys. 2020, 152, 184101.

(40) Zhang, Y.; Suo, B.; Wang, Z.; Zhang, N.; Li, Z.; Lei, Y.; Zou, W.; Gao, J.; Peng, D.; Pu, Z.; Xiao, Y.; Sun, Q.; Wang, F.; Ma, Y.; Wang, X.; Guo, Y.; Liu, W. BDF: A relativistic electronic structure program package. J. Chem. Phys. 2020, 152, 064113.

(41) Saue, T.; Bast, R.; Gomes, A. S. P.; Jensen, H. J. A.; Visscher, L.; Aucar, I. A.; Di Remigio, R.; Dyall, K. G.; Eliav, E.; Fasshauer, E.; Fleig, T.; Halbert, L.; Hedegård, E. D.; Helmich-Paris, B.; Iliaš, M.; Jacob, C. R.; Knecht, S.; Laerdahl, J. K.; Vidal, M. L.; Nayak, M. K.; Olejniczak, M.; Olsen, J. M. H.; Pernpointner, M.; Senjean, B.; Shee, A.; Sunaga, A.; van Stralen, J. N. P. The DIRAC code for relativistic molecular calculations. J. Chem. Phys. 2020, 152, 204104.

(42) Williams-Young, D. B.; Petrone, A.; Sun, S.; Stetina, T. F.; Lestrange, P.; Hoyer, C. E.; Nascimento, D. R.; Koulias, L.; Wildman, A.; Kasper, J.; Goings, J. J.; Ding, F.; 
DePrince III, A. E.; Valeev, E. F.; Li, X. The Chronus Quantum software package. Wiley Interdiscip. Rev.: Comput. Mol. Sci. 2020, 10, e1436.

(43) Werner, H.-J.; Knowles, P. J.; Manby, F. R.; Black, J. A.; Doll, K.; Heßelmann, A.; Kats, D.; Köhn, A.; Korona, T.; Kreplin, D. A.; Ma, Q.; Miller, T. F.; Mitrushchenkov, A.; Peterson, K. A.; Polyak, I.; Rauhut, G.; Sibaev, M. The Molpro quantum chemistry package. J. Chem. Phys. 2020, 152, 144107.

(44) Aquilante, F.; Autschbach, J.; Carlson, R. K.; Chibotaru, L. F.; Delcey, M. G.; De Vico, L.; Fdez. Galván, I.; Ferré, N.; Frutos, L. M.; Gagliardi, L.; Garavelli, M.; Giussani, A.; Hoyer, C. E.; Li Manni, G.; Lischka, H.; Ma, D.; Malmqvist, P. A.; Müller, T.; Nenov, A.; Olivucci, M.; Pedersen, T. B.; Peng, D.; Plasser, F.; Pritchard, B.; Reiher, M.; Rivalta, I.; Schapiro, I.; Segarra-Martí, J.; Stenrup, M.; Truhlar, D. G.; Ungur, L.; Valentini, A.; Vancoillie, S.; Veryazov, V.; Vysotskiy, V. P.; Weingart, O.; Zapata, F.; Lindh, R. Molcas 8: New capabilities for multiconfigurational quantum chemical calculations across the periodic table. J. Comput. Chem. 2016, 3\%, 506-.541.

(45) Matthews, D. A.; Cheng, L.; Harding, M. E.; Lipparini, F.; Stopkowicz, S.; Jagau, T.C.; Szalay, P. G.; Gauss, J.; Stanton, J. F. Coupled-cluster techniques for computational chemistry: The CFOUR program package. J. Chem. Phys. 2020, 152, 214108.

(46) Smith, D. G. A.; Burns, L. A.; Simmonett, A. C.; Parrish, R. M.; Schieber, M. C.; Galvelis, R.; Kraus, P.; Kruse, H.; Di Remigio, R.; Alenaizan, A.; James, A. M.; Lehtola, S.; Misiewicz, J. P.; Scheurer, M.; Shaw, R. A.; Schriber, J. B.; Xie, Y.; Glick, Z. L.; Sirianni, D. A.; O’Brien, J. S.; Waldrop, J. M.; Kumar, A.; Hohenstein, E. G.; Pritchard, B. P.; Brooks, B. R.; Schaefer, H. F.; Sokolov, A. Y.; Patkowski, K.; DePrince, A. E.; Bozkaya, U.; King, R. A.; Evangelista, F. A.; Turney, J. M.; Crawford, T. D.; Sherrill, C. D. PSI4 1.4: Open-source software for highthroughput quantum chemistry. J. Phys. Chem. 2020, 152, 184108. 
(47) Aprà, E.; Bylaska, E. J.; de Jong, W. A.; Govind, N.; Kowalski, K.; Straatsma, T. P.; Valiev, M.; van Dam, H. J. J.; Alexeev, Y.; Anchell, J.; Anisimov, V.; Aquino, F. W.; Atta-Fynn, R.; Autschbach, J.; Bauman, N. P.; Becca, J. C.; Bernholdt, D. E.; Bhaskaran-Nair, K.; Bogatko, S.; Borowski, P.; Boschen, J.; Brabec, J.; Bruner, A.; Cauët, E.; Chen, Y.; Chuev, G. N.; Cramer, C. J.; Daily, J.; Deegan, M. J. O.; Dunning, T. H.; Dupuis, M.; Dyall, K. G.; Fann, G. I.; Fischer, S. A.; Fonari, A.; Früchtl, H.; Gagliardi, L.; Garza, J.; Gawande, N.; Ghosh, S.; Glaesemann, K.; Götz, A. W.; Hammond, J.; Helms, V.; Hermes, E. D.; Hirao, K.; Hirata, S.; Jacquelin, M.; Jensen, L.; Johnson, B. G.; Jónsson, H.; Kendall, R. A.; Klemm, M.; Kobayashi, R.; Konkov, V.; Krishnamoorthy, S.; Krishnan, M.; Lin, Z.; Lins, R. D.; Littlefield, R. J.; Logsdail, A. J.; Lopata, K.; Ma, W.; Marenich, A. V.; Martin del Campo, J.; Mejia-Rodriguez, D.; Moore, J. E.; Mullin, J. M.; Nakajima, T.; Nascimento, D. R.; Nichols, J. A.; Nichols, P. J.; Nieplocha, J.; Otero-de-la Roza, A.; Palmer, B.; Panyala, A.; Pirojsirikul, T.; Peng, B.; Peverati, R.; Pittner, J.; Pollack, L.; Richard, R. M.; Sadayappan, P.; Schatz, G. C.; Shelton, W. A.; Silverstein, D. W.; Smith, D. M. A.; Soares, T. A.; Song, D.; Swart, M.; Taylor, H. L.; Thomas, G. S.; Tipparaju, V.; Truhlar, D. G.; Tsemekhman, K.; Van Voorhis, T.; Vázquez-Mayagoitia, A.; Verma, P.; Villa, O.; Vishnu, A.; Vogiatzis, K. D.; Wang, D.; Weare, J. H.; Williamson, M. J.; Windus, T. L.; Woliński, K.; Wong, A. T.; Wu, Q.; Yang, C.; Yu, Q.; Zacharias, M.; Zhang, Z.; Zhao, Y.; Harrison, R. J. NWChem: Past, present, and future. J. Chem. Phys. 2020, 152, 184102.

(48) Balasubramani, S. G.; Chen, G. P.; Coriani, S.; Diedenhofen, M.; Frank, M. S.; Franzke, Y. J.; Furche, F.; Grotjahn, R.; Harding, M. E.; Hättig, C.; Hellweg, A.; Helmich-Paris, B.; Holzer, C.; Huniar, U.; Kaupp, M.; Marefat Khah, A.; Karbalaei Khani, S.; Müller, T.; Mack, F.; Nguyen, B. D.; Parker, S. M.; Perlt, E.; Rappoport, D.; Reiter, K.; Roy, S.; Rückert, M.; Schmitz, G.; Sierka, M.; Tapavicza, E.; Tew, D. P.; van Wüllen, C.; Voora, V. K.; Weigend, F.; Wodyński, A.; Yu, J. M. TUR- 
BOMOLE: Modular program suite for ab initio quantum-chemical and condensedmatter simulations. J. Chem. Phys. 2020, 152, 184107.

(49) Cheng, L.; Stopkowicz, S.; Gauss, J. Analytic energy derivatives in relativistic quantum chemistry. Int. J. Quantum Chem. 2014, 114, 1108-1127.

(50) Cremer, D.; Zou, W.; Filatov, M. Dirac-exact relativistic methods: the normalized elimination of the small component method. Wiley Interdiscip. Rev.: Comput. Mol. Sci. 2014, 4, 436-467.

(51) Nakajima, T.; Hirao, K. The Douglas-Kroll-Hess Approach. Chem. Rev. 2012, 112, 385-402.

(52) Reiher, M. Relativistic Douglas-Kroll-Hess theory. Wiley Interdiscip. Rev.: Comput. Mol. Sci. 2012, 2, 139-149.

(53) Zou, W.; Filatov, M.; Cremer, D. Development and application of the analytical energy gradient for the normalized elimination of the small component method. J. Chem. Phys. 2011, 134, 244117.

(54) Cheng, L.; Gauss, J. Analytic energy gradients for the spin-free exact two-component theory using an exact block diagonalization for the one-electron Dirac Hamiltonian. J. Chem. Phys. 2011, 135, 084114.

(55) Cheng, L.; Gauss, J. Analytic second derivatives for the spin-free exact two-component theory. J. Chem. Phys. 2011, 135, 244104.

(56) Zou, W.; Filatov, M.; Cremer, D. Development, Implementation, and Application of an Analytic Second Derivative Formalism for the Normalized Elimination of the Small Component Method. J. Chem. Theory Comput. 2012, 8, 2617-2629.

(57) Zou, W.; Filatov, M.; Cremer, D. Analytical energy gradient for the two-component 
normalized elimination of the small component method. J. Chem. Phys. 2015, 142, 214106.

(58) Franzke, Y. J.; Middendorf, N.; Weigend, F. Efficient implementation of one- and two-component analytical energy gradients in exact two-component theory. J. Chem. Phys. 2018, 148, 104110.

(59) Zou, W.; Guo, G.; Suo, B.; Liu, W. Analytic Energy Gradients and Hessians of Exact Two-Component Relativistic Methods: Efficient Implementation and Extensive Applications. J. Chem. Theory Comput. 2020, 16, 1541-1554.

(60) Filatov, M.; Zou, W.; Cremer, D. Analytic Calculation of Contact Densities and Mössbauer Isomer Shifts Using the Normalized Elimination of the Small-Component Formalism. J. Chem. Theory Comput. 2012, 8, 875-882.

(61) Yoshizawa, T.; Zou, W.; Cremer, D. Calculations of electric dipole moments and static dipole polarizabilities based on the two-component normalized elimination of the small component method. J. Chem. Phys. 2016, 145, 184104.

(62) Yoshizawa, T.; Filatov, M.; Cremer, D.; Zou, W. Calculation of contact densities and Mössbauer isomer shifts utilising the Dirac-exact two-component normalised elimination of the small component (2c-NESC) method. Mol. Phys. 2019, 117, 1164-1171.

(63) Zhu, H.; Gao, C.; Filatov, M.; Zou, W. Mössbauer isomer shifts and effective contact densities obtained by the exact two-component (X2C) relativistic method and its local variants. Phys. Chem. Chem. Phys. 2020, 22, 26776-26786.

(64) Sun, Q.; Liu, W.; Xiao, Y.; Cheng, L. Exact two-component relativistic theory for nuclear magnetic resonance parameters. J. Chem. Phys. 2009, 131, 081101.

(65) Sun, Q.; Xiao, Y.; Liu, W. Exact two-component relativistic theory for NMR parameters: General formulation and pilot application. J. Chem. Phys. 2012, 137, 174105. 
(66) Cheng, L.; Gauss, J.; Stanton, J. F. Treatment of scalar-relativistic effects on nuclear magnetic shieldings using a spin-free exact-two-component approach. J. Chem. Phys. 2013, 139, 054105 .

(67) Yoshizawa, T.; Zou, W.; Cremer, D. Calculations of atomic magnetic nuclear shielding constants based on the two-component normalized elimination of the small component method. J. Chem. Phys. 2017, 146, 134109.

(68) Yoshizawa, T.; Hada, M. Calculations of nuclear magnetic shielding constants based on the exact two-component relativistic method. J. Chem. Phys. 2017, 147, 154104.

(69) Yoshizawa, T. On the development of the exact two-component relativistic method for calculating indirect NMR spin-spin coupling constants. Chem. Phys. 2019, 518, $112-122$.

(70) Franzke, Y. J.; Weigend, F. NMR Shielding Tensors and Chemical Shifts in ScalarRelativistic Local Exact Two-Component Theory. J. Chem. Theory Comput. 2019, 15, 1028-1043.

(71) Franzke, Y. J.; Mack, F.; Weigend, F. NMR Indirect Spin-Spin Coupling Constants in a Modern Quasirelativistic Density Functional Framework. J. Chem. Theory Comput. 2021, 17, 3974-3994.

(72) Filatov, M.; Zou, W.; Cremer, D. Analytic Calculation of Isotropic Hyperfine Structure Constants Using the Normalized Elimination of the Small Component Formalism. J. Phys. Chem. A 2012, 116, 3481-3486.

(73) Franzke, Y. J.; Yu, J. M. Hyperfine Coupling Constants in Local Exact TwoComponent Theory. ChemRxiv 2021, DOI: 10.33774/chemrxiv-2021-wnz1v-v2.

(74) Gillhuber, S.; Franzke, Y. J.; Weigend, F. Paramagnetic NMR Shielding Tensors and 
Ring Currents: Efficient Implementation and Application to Heavy Element Compounds. J. Phys. Chem. A 2021, 125, 9707-9723.

(75) Autschbach, J. Relativistic Effects on Electron-Nucleus Hyperfine Coupling Studied with an Exact 2-Component (X2C) Hamiltonian. J. Chem. Theory Comput. 2017, $13,710-718$.

(76) Feng, R.; Duignan, T. J.; Autschbach, J. Electron-Nucleus Hyperfine Coupling Calculated from Restricted Active Space Wavefunctions and an Exact Two-Component Hamiltonian. J. Chem. Theory Comput. 2021, 17, 255-268.

(77) Wodyński, A.; Kaupp, M. Density Functional Calculations of EPR g- and HyperfineCoupling Tensors Using the Exact Two-Component (X2C) Transformation and Efficient Approximations to the Two-Electron Spin-Orbit Terms. J. Phys. Chem. A 2019, 123, 5660-5672.

(78) Stanton, R. E.; Havriliak, S. Kinetic balance: A partial solution to the problem of variational safety in Dirac calculations. J. Chem. Phys. 1984, 81, 1910-1918.

(79) Komorovský, S.; Repiský, M.; Malkina, O. L.; Malkin, V. G.; Malkin Ondík, I.; Kaupp, M. A fully relativistic method for calculation of nuclear magnetic shielding tensors with a restricted magnetically balanced basis in the framework of the matrix Dirac-Kohn-Sham equation. J. Chem. Phys. 2008, 128, 104101.

(80) Sun, S.; Li, X. Relativistic Effects in Magnetic Circular Dichroism: Restricted Magnetic Balance and Temperature Dependence. J. Chem. Theory Comput. 2020, 16, $4533-4542$.

(81) Kutzelnigg, W. Ab initio calculation of molecular properties. J. Mol. Struct.: THEOCHEM 1989, 202, 11-61. 
(82) London, F. Théorie quantique des courants interatomiques dans les combinaisons aromatiques. J. Phys. Radium 1937, 8, 397-409.

(83) Ditchfield, R. Self-consistent perturbation theory of diamagnetism. Mol. Phys. 1974, $27,789-807$.

(84) Komorovský, S.; Repiský, M.; Malkina, O. L.; Malkin, V. G. Fully relativistic calculations of NMR shielding tensors using restricted magnetically balanced basis and gauge including atomic orbitals. J. Chem. Phys. 2010, 132, 154101.

(85) Olejniczak, M.; Bast, R.; Saue, T.; Pecul, M. A simple scheme for magnetic balance in four-component relativistic Kohn-Sham calculations of nuclear magnetic resonance shielding constants in a Gaussian basis. J. Chem. Phys. 2012, 136, 014108.

(86) Olejniczak, M.; Bast, R.; Saue, T.; Pecul, M. Erratum: "A simple scheme for magnetic balance in four-component relativistic Kohn-Sham calculations of nuclear magnetic resonance shielding constants in a Gaussian basis" [J. Chem. Phys. 136, 014108 (2012)]. J. Chem. Phys. 2012, 136, 239902.

(87) Fukuda, R.; Hada, M.; Nakatsuji, H. Quasirelativistic theory for magnetic shielding constants. II. Gauge-including atomic orbitals and applications to molecules. J. Phys. Chem. 2003, 118, 1027-1035.

(88) Yoshizawa, T.; Hada, M. Gauge-origin dependence of NMR shielding constants in the Douglas-Kroll-Hess method. Chem. Phys. Lett. 2015, 618, 132-141.

(89) Hayami, M.; Seino, J.; Nakai, H. Gauge-origin independent formalism of twocomponent relativistic framework based on unitary transformation in nuclear magnetic shielding constant. J. Chem. Phys. 2018, 148, 114109.

(90) Wolff, S. K.; Ziegler, T.; van Lenthe, E.; Baerends, E. J. Density functional calculations of nuclear magnetic shieldings using the zeroth-order regular approximation (ZORA) 
for relativistic effects: ZORA nuclear magnetic resonance. J. Chem. Phys. 1999, 110, 7689-7698.

(91) Autschbach, J. The role of the exchange-correlation response kernel and scaling corrections in relativistic density functional nuclear magnetic shielding calculations with the zeroth-order regular approximation. Mol. Phys. 2013, 111, 2544-2554.

(92) Neese, F.; Solomon, E. I. Calculation of Zero-Field Splittings, g-Values, and the Relativistic Nephelauxetic Effect in Transition Metal Complexes. Application to High-Spin Ferric Complexes. Inorg. Chem. 1998, 37, 6568-6582.

(93) Tran, V. A.; Neese, F. Double-hybrid density functional theory for g-tensor calculations using gauge including atomic orbitals. J. Chem. Phys. 2020, 153, 054105.

(94) Schreckenbach, G.; Ziegler, T. Calculation of the G-Tensor of Electron Paramagnetic Resonance Spectroscopy Using Gauge-Including Atomic Orbitals and Density Functional Theory. J. Phys. Chem. A 1997, 101, 3388-3399.

(95) Schreckenbach, G.; Ziegler, T. Density functional calculations of NMR chemical shifts and ESR g-tensors. Theor. Chem. Acc. 1998, 99, 71-82.

(96) Patchkovskii, S.; Ziegler, T. Prediction of electron paramagnetic resonance g-tensors of transition metal complexes using density functional theory: First applications to some axial $d^{1} \mathrm{MEX}_{4}$ systems. J. Phys. Chem. 1999, 111, 5730-5740.

(97) Patchkovskii, S.; Ziegler, T. Prediction of EPR g Tensors in Simple $d^{1}$ Metal Porphyrins with Density Functional Theory. J. Am. Chem. Soc. 2000, 122, 3506-3516.

(98) Patchkovskii, S.; Strong, R. T.; Pickard, C. J.; Un, S. Gauge invariance of the spinother-orbit contribution to the g-tensors of electron paramagnetic resonance. J. Chem. Phys. 2005, 122, 214101. 
(99) Peng, D.; Reiher, M. Local relativistic exact decoupling. J. Chem. Phys. 2012, 136, 244108.

(100) Zhang, T.; Kasper, J. M.; Li, X. Localized relativistic two-component methods for ground and excited state calculations. In Annual Reports in Computational Chemistry; Dixon, D. A., Ed.; Elsevier: Amsterdam, The Netherlands, 2020; Vol. 16; Chapter 2, pp $17-37$.

(101) Peng, D.; Middendorf, N.; Weigend, F.; Reiher, M. An efficient implementation of two-component relativistic exact-decoupling methods for large molecules. J. Chem. Phys. 2013, 138, 184105.

(102) Seino, J.; Nakai, H. Local unitary transformation method for large-scale twocomponent relativistic calculations: Case for a one-electron Dirac Hamiltonian. J. Chem. Phys. 2012, 136, 244102.

(103) Seino, J.; Nakai, H. Local unitary transformation method for large-scale twocomponent relativistic calculations. II. Extension to two-electron Coulomb interaction. J. Chem. Phys. 2012, 137, 144101.

(104) Lichtenberger, N.; Franzke, Y. J.; Massa, W.; Weigend, F.; Dehnen, S. The Identity of "Ternary" $\mathrm{A} / \mathrm{Tl} / \mathrm{Pb}$ or $\mathrm{K} / \mathrm{Tl} / \mathrm{Bi}$ Solid Mixtures and Binary Zintl Anions Isolated From Their Solutions. Chem. Eur. J. 2018, 24, 12022-12030.

(105) Balmer, M.; Franzke, Y. J.; Weigend, F.; von Hänisch, C. Low valent group 14 phosphinidenide complexes $\left[(\operatorname{SIDippP})_{2} \mathrm{M}\right]$ exhibit P-M $\mathrm{p} \pi$-p $\pi$ interaction $(\mathrm{M}=\mathrm{Ge}, \mathrm{Sn}$, Pb). Chem. Eur. J. 2020, 26, 192-197.

(106) Eulenstein, A. R.; Franzke, Y. J.; Bügel, P.; Massa, W.; Weigend, F.; Dehnen, S. Stabilizing a metalloid $\left\{\mathrm{Zn}_{12}\right\}$ unit within a polymetallide environment in $\left[\mathrm{K}_{2} \mathrm{Zn}_{20} \mathrm{Bi}_{16}\right]^{6-}$. Nat. Commun. 2020, 11, 5122. 
(107) Eulenstein, A. R.; Franzke, Y. J.; Lichtenberger, N.; Wilson, R. J.; Deubner, H. L.; Kraus, F.; Weigend, F.; Dehnen, S. Substantial $\pi$-aromaticity of the anionic heavymetal cluster $\left[\mathrm{Th} @ \mathrm{Bi}_{12}\right]^{4-}$. Nat. Chem. 2021, 13, 149-155.

(108) Van Wüllen, C. Spin densities in two-component relativistic density functional calculations: Noncollinear versus collinear approach. J. Comput. Chem. 2002, 23, 779-785.

(109) Verma, P.; Autschbach, J. Relativistic Density Functional Calculations of Hyperfine Coupling with Variational versus Perturbational Treatment of Spin-Orbit Coupling. J. Chem. Theory Comput. 2013, 9, 1932-1948.

(110) Gell-Mann, M. The interpretation of the new particles as displaced charge multiplets. Nuovo Cim. 1956, 4, 848-866.

(111) Foldy, L. L.; Wouthuysen, S. A. On the Dirac Theory of Spin 1/2 Particles and Its Non-Relativistic Limit. Phys. Rev. 1950, 78, 29-36.

(112) Heully, J.-L.; Lindgren, I.; Lindroth, E.; Lundqvist, S.; Mårtensson-Pendrill, A.-M. Diagonalisation of the Dirac Hamiltonian as a basis for a relativistic many-body procedure. J. Phys. B: At. Mol. Phys. 1986, 19, 2799-2815.

(113) Dyall, K. G. Interfacing relativistic and nonrelativistic methods. I. Normalized elimination of the small component in the modified Dirac equation. J. Chem. Phys. 1997, $106,9618-9626$.

(114) Dyall, K. G. Interfacing relativistic and nonrelativistic methods. II. Investigation of a low-order approximation. J. Chem. Phys. 1998, 109, 4201-4208.

(115) Dyall, K. G.; Enevoldsen, T. Interfacing relativistic and nonrelativistic methods. III. Atomic 4-spinor expansions and integral approximations. J. Chem. Phys. 1999, 111, 10000-10007. 
(116) Dyall, K. G. Interfacing relativistic and nonrelativistic methods. IV. One- and twoelectron scalar approximations. J. Chem. Phys. 2001, 115, 9136-9143.

(117) Boettger, J. C. Approximate two-electron spin-orbit coupling term for densityfunctional-theory DFT calculations using the Douglas-Kroll-Hess transformation. Phys. Rev. B 2000, 62, 7809-7815.

(118) Filatov, M.; Zou, W.; Cremer, D. Spin-orbit coupling calculations with the twocomponent normalized elimination of the small component method. J. Chem. Phys. 2013, 139, 014106.

(119) Pulay, P. Ab initio calculation of force constants and equilibrium geometries in polyatomic molecules. Mol. Phys. 1969, 17, 197-204.

(120) Rice, J.; Amos, R. On the efficient evaluation of analytic energy gradients. Chem. Phys. Lett. 1985, 122, 585-590.

(121) Baldes, A.; Weigend, F. Efficient two-component self-consistent field procedures and gradients: implementation in TURBOMOLE and application to $\mathrm{Au}_{20}^{-}$. Mol. Phys. 2013, 111, 2617-2624.

(122) Pausch, A.; Klopper, W. Efficient evaluation of three-centre two-electron integrals over London orbitals. Mol. Phys. 2020, 118, e1736675.

(123) Wolinski, K.; Hinton, J. F.; Pulay, P. Efficient implementation of the gaugeindependent atomic orbital method for NMR chemical shift calculations. J. Am. Chem. Soc. 1990, 112, 8251-8260.

(124) Häser, M.; Ahlrichs, R.; Baron, H. P.; Weis, P.; Horn, H. Direct computation of secondorder scf properties of large molecules on workstation computers with an application to large carbon clusters. Theor. Chim. Acta 1992, 83, 455-470. 
(125) Reiter, K.; Mack, F.; Weigend, F. Calculation of Magnetic Shielding Constants with meta-GGA Functionals Employing the Multipole-Accelerated Resolution of the Identity: Implementation and Assessment of Accuracy and Efficiency. J. Chem.Theory Comput. 2018, 14, 191-197.

(126) Armbruster, M. K.; Weigend, F.; van Wüllen, C.; Klopper, W. Self-consistent treatment of spin-orbit interactions with efficient Hartree-Fock and density functional methods. Phys. Chem. Chem. Phys. 2008, 10, 1748-1756.

(127) Krykunov, M.; Ziegler, T.; van Lenthe, E. Implementation of a Hybrid DFT Method for Calculating NMR Shieldings Using Slater-Type Orbitals with Spin-Orbital Coupling Included. Applications to ${ }^{187} \mathrm{Os},{ }^{195} \mathrm{Pt}$, and ${ }^{13} \mathrm{C}$ in Heavy-Metal Complexes. J. Phys. Chem. A 2009, 113, 11495-11500.

(128) Franzke, Y. J. Calculation of NMR Parameters in a Modern Relativistic Density Functional Framework: Theory, Implementation, and Application. Dissertation, Karlsruhe Institute of Technology (KIT), Germany, 2021.

(129) van Wüllen, C.; Michauk, C. Accurate and efficient treatment of two-electron contributions in quasirelativistic high-order Douglas-Kroll density-functional calculations. J. Chem. Phys. 2005, 123, 204113.

(130) Plessow, P.; Weigend, F. Seminumerical calculation of the Hartree-Fock exchange matrix: Application to two-component procedures and efficient evaluation of local hybrid density functionals. J. Comput. Chem. 2012, 33, 810-816.

(131) Holzer, C. An improved seminumerical Coulomb and exchange algorithm for properties and excited states in modern density functional theory. J. Chem. Phys. 2020, 153, 184115.

(132) Pollak, P.; Weigend, F. Segmented Contracted Error-Consistent Basis Sets of Double- 
and Triple- $\zeta$ Valence Quality for One- and Two-Component Relativistic All-Electron Calculations. J. Chem. Theory Comput. 2017, 13, 3696-3705.

(133) Ahlrichs, R.; Bär, M.; Häser, M.; Horn, H.; Kölmel, C. Electronic structure calculations on workstation computers: The program system turbomole. Chem. Phys. Lett. 1989, 162, 165-169.

(134) Furche, F.; Ahlrichs, R.; Hättig, C.; Klopper, W.; Sierka, M.; Weigend, F. Turbomole. Wiley Interdiscip. Rev.: Comput. Mol. Sci. 2014, 4, 91-100.

(135) Developers' version of TURBOMOLE V7.5.1 2021, a development of University of Karlsruhe and Forschungszentrum Karlsruhe GmbH, 1989-2007, TURBOMOLE GmbH, since 2007; available from https://www.turbomole.org (retrieved August $23,2021)$.

(136) Huniar, U. Berechnung der chemischen Verschiebung der NMR mit Methoden der Dichtefunktionaltheorie (DFT). Diploma Thesis, University of Karlsruhe (TH), Germany, 1999.

(137) Kollwitz, M.; Gauss, J. A direct implementation of the GIAO-MBPT(2) method for calculating NMR chemical shifts. Application to the naphthalenium and anthracenium ions. Chem. Phys. Lett. 1996, 260, 639-646.

(138) Kollwitz, M.; Häser, M.; Gauss, J. Non-Abelian point group symmetry in direct second-order many-body perturbation theory calculations of NMR chemical shifts. J. Chem. Phys. 1998, 108, 8295-8301.

(139) Dupuis, M.; Rys, J.; King, H. F. Evaluation of molecular integrals over Gaussian basis functions. J. Chem. Phys. 1976, 65, 111-116.

(140) Rys, J.; Dupuis, M.; King, H. F. Computation of electron repulsion integrals using the rys quadrature method. J. Comput. Chem. 1983, 4, 154-157. 
(141) Dupuis, M.; Marquez, A. The Rys quadrature revisited: A novel formulation for the efficient computation of electron repulsion integrals over Gaussian functions. J. Chem. Phys. 2001, 114, 2067-2078.

(142) Gautschi, W. Algorithm 726: ORTHPOL-a Package of Routines for Generating Orthogonal Polynomials and Gauss-Type Quadrature Rules. ACM Trans. Math. Softw. 1994, 20, 21-62.

(143) Kehry, M.; Franzke, Y. J.; Holzer, C.; Klopper, W. Quasirelativistic two-component core excitations and polarisabilities from a damped-response formulation of the BetheSalpeter equation. Mol. Phys. 2020, 118, e1755064.

(144) Visscher, L.; Dyall, K. G. Dirac-Fock atomic electronic structure calculations using different nuclear charge distributions. At. Data Nucl. Data Tables 1997, 67, 207-224.

(145) CODATA internationally recommened 2018 values of the fundamental physical constans, https://physics.nist.gov/cuu/Constants/index.html (retrieved December 28, 2020).

(146) Klamt, A.; Schüürmann, G. COSMO: a new approach to dielectric screening in solvents with explicit expressions for the screening energy and its gradient. J. Chem. Soc., Perkin Trans. 2 1993, 799-805.

(147) Schäfer, A.; Klamt, A.; Sattel, D.; Lohrenz, J. C. W.; Eckert, F. COSMO Implementation in TURBOMOLE: Extension of an efficient quantum chemical code towards liquid systems. Phys. Chem. Chem. Phys. 2000, 2, 2187-2193.

(148) Weigend, F. A fully direct RI-HF algorithm: Implementation, optimised auxiliary basis sets, demonstration of accuracy and efficiency. Phys. Chem. Chem. Phys. 2002, 4, 4285-4291. 
(149) Perdew, J. P.; Schmidt, K. Jacob's ladder of density functional approximations for the exchange-correlation energy. AIP Conf. Proc. 2001, 57r, 1-20.

(150) Becke, A. D. Perspective: Fifty years of density-functional theory in chemical physics. J. Chem. Phys. 2014, 140, 18A301.

(151) Mardirossian, N.; Head-Gordon, M. Thirty years of density functional theory in computational chemistry: an overview and extensive assessment of 200 density functionals. Mol. Phys. 2017, 115, 2315-2372.

(152) Maximoff, S. N.; Scuseria, G. E. Nuclear magnetic resonance shielding tensors calculated with kinetic energy density-dependent exchange-correlation functionals. Chem. Phys. Lett. 2004, 390, 408-412.

(153) Marques, M. A. L.; Oliveira, M. J. T.; Burnus, T. Libxc: A library of exchange and correlation functionals for density functional theory. Comput. Phys. Commun. 2012, 183, 2272-2281.

(154) Lehtola, S.; Steigemann, C.; Oliveira, M. J. T.; Marques, M. A. L. Recent developments in libxc - A comprehensive library of functionals for density functional theory. SoftwareX 2018, 7, 1-5.

(155) Libxc. 5.1.4, available from https://www.tddft.org/programs/libxc/ (retrieved July 30, 2021).

(156) Ekström, U.; Visscher, L.; Bast, R.; Thorvaldsen, A. J.; Ruud, K. Arbitrary-Order Density Functional Response Theory from Automatic Differentiation. J. Chem. Theory Comput. 2010, 6, 1971-1980.

(157) Reiter, K. Weiterentwicklung und Anwendung von quantenchemischen Methoden zur Berechnung von Molekülen im Magnetfeld. Dissertation, Karlsruhe Institute of Technology (KIT), Germany, 2018. 
(158) Van Wüllen, C. Relativistic all-electron density functional calculations. J. Comput. Chem. 1999, 20, 51-62.

(159) OpenMP Architecture Review Boards, OpenMP API shared-memory parallel programming. https: //www . openmp.org (retrieved December 4, 2020).

(160) Holzer, C.; Franzke, Y. J. OpenMP version of ridft, rdgrad, and egrad with contributions to mpshift, dscf, and grad; improved OpenMP version of aoforce and escf, released with TURBOMOLE V7.4 and further improved in TURBOMOLE V7.5.

(161) Belanzoni, P.; van Lenthe, E.; Baerends, E. J. An evaluation of the density functional approach in the zero order regular approximation for relativistic effects: Magnetic interactions in small metal compounds. J. Chem. Phys. 2001, 114, 4421-4433.

(162) Perdew, J. P. Density-functional approximation for the correlation energy of the inhomogeneous electron gas. Phys. Rev. B 1986, 33, 8822-8824.

(163) Becke, A. D. Density-functional exchange-energy approximation with correct asymptotic behavior. Phys. Rev. A 1988, 38, 3098-3100.

(164) Franzke, Y. J.; Spiske, L.; Pollak, P.; Weigend, F. Segmented Contracted ErrorConsistent Basis Sets of Quadruple- $\zeta$ Valence Quality for One- and Two-Component Relativistic All-Electron Calculations. J. Chem. Theory Comput. 2020, 16, 5658-5674.

(165) Dyall, K. G. Relativistic Quadruple-Zeta and Revised Triple-Zeta and Double-Zeta Basis Sets for the 4p, 5p, and 6p Elements. Theor. Chem. Acc. 2006, 115, 441-447.

(166) Dyall, K. G. Relativistic double-zeta, triple-zeta, and quadruple-zeta basis sets for the actinides Ac-Lr. Theor. Chem. Acc. 2007, 117, 491-500.

(167) Gomes, A. S. P.; Dyall, K. G.; Visscher, L. Relativistic double-zeta, triple-zeta, and quadruple-zeta basis sets for the lanthanides La-Lu. Theor. Chem. Acc. 2010, 127, $369-381$. 
(168) Basis sets available from the Dirac program web site, http://dirac.chem.sdu.dk (retrieved December 4, 2020).

(169) Jensen, F. Basis Set Convergence of Nuclear Magnetic Shielding Constants Calculated by Density Functional Methods. J. Chem. Theory Comput. 2008, 4, 719-727.

(170) Jensen, F. Segmented Contracted Basis Sets Optimized for Nuclear Magnetic Shielding. J. Chem. Theory Comput. 2015, 11, 132-138.

(171) Perdew, J. P.; Burke, K.; Ernzerhof, M. Generalized Gradient Approximation Made Simple. Phys. Rev. Lett. 1996, r7, 3865-3868.

(172) Adamo, C.; Barone, V. Toward reliable density functional methods without adjustable parameters: The PBE0 model. J. Chem. Phys. 1999, 110, 6158-6170.

(173) Chai, J.-D.; Head-Gordon, M. Long-range corrected hybrid density functionals with damped atom-atom dispersion corrections. Phys. Chem. Chem. Phys. 2008, 10, 66156620.

(174) Treutler, O.; Ahlrichs, R. Efficient molecular numerical integration schemes. J. Chem. Phys. 1995, 102, 346-354.

(175) Treutler, O. Entwicklung und Anwendung von Dichtefunktionalmethoden. Ph.D. thesis, University of Karlsruhe (TH), Germany, 1995.

(176) Franzke, Y. J.; Treß, R.; Pazdera, T. M.; Weigend, F. Error-consistent segmented contracted all-electron relativistic basis sets of double- and triple-zeta quality for NMR shielding constants. Phys. Chem. Chem. Phys. 2019, 21, 16658-16664.

(177) Kutzelnigg, W.; Fleischer, U.; Schindler, M. The IGLO-Method: Ab-initio Calculation and Interpretation of NMR Chemical Shifts and Magnetic Susceptibilities. In Deuterium and Shift Calculation; Springer: Berlin, Heidelberg, Germany, 1991; pp $165-262$. 
(178) Noro, T.; Sekiya, M.; Koga, T. Segmented contracted basis sets for atoms H through Xe: Sapporo-(DK)-nZP sets (n = D, T, Q). Theor. Chem. Acc. 2012, 131, 1124.

(179) Noro, T.; Sekiya, M.; Koga, T. Sapporo-(DKH3)-nZP (n = D, T, Q) sets for the sixth period s-, d-, and p-block atoms. Theor. Chem. Acc. 2013, 132, 1363.

(180) Dunning, T. H. Gaussian basis sets for use in correlated molecular calculations. I. The atoms boron through neon and hydrogen. J. Chem. Phys. 1989, 90, 1007-1023.

(181) Woon, D. E.; Dunning, T. H. Gaussian basis sets for use in correlated molecular calculations. III. The atoms aluminum through argon. J. Chem. Phys. 1993, 98, 13581371.

(182) Jorge, F. E.; Canal Neto, A.; Camiletti, G. G.; Machado, S. F. Contracted Gaussian basis sets for Douglas-Kroll-Hess calculations: Estimating scalar relativistic effects of some atomic and molecular properties. J. Chem. Phys. 2009, 130, 064108.

(183) Campos, C. T.; Jorge, F. E. Triple zeta quality basis sets for atoms Rb through Xe: application in CCSD(T) atomic and molecular property calculations. Mol. Phys. 2013, $111,167-173$.

(184) Martins, L.; Jorge, F.; Machado, S. All-electron segmented contraction basis sets of triple zeta valence quality for the fifth-row elements. Mol. Phys. 2015, 113, 3578-3586.

(185) Zobel, J. P.; Widmark, P.-O.; Veryazov, V. The ANO-R Basis Set. J. Chem. Theory Comput. 2020, 16, 278-294.

(186) Roos, B. O.; Lindh, R.; Malmqvist, P.-A.; Veryazov, V.; Widmark, P.-O. Main Group Atoms and Dimers Studied with a New Relativistic ANO Basis Set. J. Phys. Chem. A 2004, 108, 2851-2858. 
(187) Roos, B. O.; Lindh, R.; Malmqvist, P.-Å.; Veryazov, V.; Widmark, P.-O. New Relativistic ANO Basis Sets for Transition Metal Atoms. J. Phys. Chem. A 2005, 109, $6575-6579$.

(188) Dirac, P. A. M. Quantum Mechanics of Many-Electron Systems. Proc. Roy. Soc. Lond. A 1929, 123, 714-733.

(189) Slater, J. C. A Simplification of the Hartree-Fock Method. Phys. Rev. 1951, 81, 385390.

(190) Vosko, S. H.; Wilk, L.; Nusair, M. Accurate spin-dependent electron liquid correlation energies for local spin density calculations: a critical analysis. Can. J. Phys. 1980, $58,1200-1211$.

(191) Keal, T. W.; Tozer, D. J. A semiempirical generalized gradient approximation exchange-correlation functional. J. Chem. Phys. 2004, 121, 5654-5660.

(192) Tao, J.; Perdew, J. P.; Staroverov, V. N.; Scuseria, G. E. Climbing the Density Functional Ladder: Nonempirical Meta-Generalized Gradient Approximation Designed for Molecules and Solids. Phys. Rev. Lett. 2003, 91, 146401.

(193) Perdew, J. P.; Ruzsinszky, A.; Csonka, G. I.; Constantin, L. A.; Sun, J. Workhorse Semilocal Density Functional for Condensed Matter Physics and Quantum Chemistry. Phys. Rev. Lett. 2009, 103, 026403.

(194) Perdew, J. P.; Ruzsinszky, A.; Csonka, G. I.; Constantin, L. A.; Sun, J. Erratum: Workhorse Semilocal Density Functional for Condensed Matter Physics and Quantum Chemistry [Phys. Rev. Lett. 103, 026403 (2009)]. Phys. Rev. Lett. 2011, 106, 179902.

(195) Furness, J. W.; Kaplan, A. D.; Ning, J.; Perdew, J. P.; Sun, J. Accurate and Numerically Efficient $\mathrm{r}^{2}$ SCAN Meta-Generalized Gradient Approximation. J. Chem. Phys. Lett. 2020, 11, 8208-8215. 
(196) Furness, J. W.; Kaplan, A. D.; Ning, J.; Perdew, J. P.; Sun, J. Correction to "Accurate and Numerically Efficient $\mathrm{r}^{2} \mathrm{SCAN}$ Meta-Generalized Gradient Approximation". J. Phys. Chem. Lett. 2020, 11, 9248-9248.

(197) Lee, C.; Yang, W.; Parr, R. G. Development of the Colle-Salvetti correlation-energy formula into a functional of the electron density. Phys. Rev. B 1988, 37, 785-789.

(198) Becke, A. D. A new mixing of Hartree-Fock and local density-functional theories. J. Chem. Phys. 1993, 98, 1372-1377.

(199) Becke, A. D. Density-functional thermochemistry. III. The role of exact exchange. J. Chem. Phys. 1993, 98, 5648-5652.

(200) Becke, A. D. Density-functional thermochemistry. V. Systematic optimization of exchange-correlation functionals. J. Phys. Chem. 1997, 107, 8554-8560.

(201) Wilson, P. J.; Bradley, T. J.; Tozer, D. J. Hybrid exchange-correlation functional determined from thermochemical data and ab initio potentials. J. Chem. Phys. 2001, $115,9233-9242$.

(202) Staroverov, V. N.; Scuseria, G. E.; Tao, J.; Perdew, J. P. Comparative assessment of a new nonempirical density functional: Molecules and hydrogen-bonded complexes. $J$. Chem. Phys. 2003, 119, 12129-12137.

(203) Grimme, S. Accurate Calculation of the Heats of Formation for Large Main Group Compounds with Spin-Component Scaled MP2 Methods. J. Phys. Chem. A 2005, 109, 3067-3077.

(204) Yanai, T.; Tew, D. P.; Handy, N. C. A new hybrid exchange-correlation functional using the Coulomb-attenuating method (CAM-B3LYP). Chem. Phys. Lett. 2004, 393, 51-57. 
(205) Jin, Y.; Bartlett, R. J. The QTP family of consistent functionals and potentials in Kohn-Sham density functional theory. J. Chem. Phys. 2016, 145, 034107.

(206) Haiduke, R. L. A.; Bartlett, R. J. Communication: Can excitation energies be obtained from orbital energies in a correlated orbital theory? J. Chem. Phys. 2018, 149, 131101.

(207) Heyd, J.; Scuseria, G. E.; Ernzerhof, M. Hybrid functionals based on a screened Coulomb potential. J. Chem. Phys. 2003, 118, 8207-8215.

(208) Heyd, J.; Scuseria, G. E.; Ernzerhof, M. Erratum: "Hybrid functionals based on a screened Coulomb potential" [J. Chem. Phys. 118, 8207 (2003)]. J. Phys. Chem. 2006, $124,219906$.

(209) Krukau, A. V.; Vydrov, O. A.; Izmaylov, A. F.; Scuseria, G. E. Influence of the exchange screening parameter on the performance of screened hybrid functionals. $J$. Phys. Chem. 2006, 125, 224106.

(210) Vydrov, O. A.; Scuseria, G. E. Assessment of a long-range corrected hybrid functional. J. Chem. Phys. 2006, 125, 234109.

(211) Schmitte, J.; Friebel, C.; Weller, F.; Dehnicke, K. Synthese, IR- und EPR-Spektren sowie die Kristallstruktur von $\left(\mathrm{PPh}_{3} \mathrm{Me}\right)_{2}\left[\mathrm{MoNCl}_{4}\right]$. Z. Anorg. Allg. Chem. 1982, 495, 148-156.

(212) Sunil, K. K.; Rogers, M. T. ESR studies of some oxotetrahalo complexes of vanadium(IV) and molybdenum(V). Inorg. Chem. 1981, 20, 3283-3287.

(213) Manoharan, P. T.; Rogers, M. T. Ligand Hyperfine Interactions in Molybdenyl and Chromyl Halide Complexes. J. Chem. Phys. 1968, 49, 5510-5519.

(214) van Kemenade, J. T. C. Ligand Hyperfine Interactions in Oxyhalides of Pentavalent Chromium, Molybdenum and Tungsten. Ph.D. thesis, Technical University Delft, The Netherlands, 1970. 
(215) Baldas, J.; Boas, J. F.; Bonnyman, J. Preparation and Properties of Nitridotechnetic (VI) Acid. I. Observation of the ESR-Spectrum of the $\left[\mathrm{TcNF}_{4}\right]^{-}$Anion in Hydrofluoric Acid Solution. Aus. J. Chem. 1989, 42, 639-648.

(216) Baldas, J.; Boas, J. F.; Bonnyman, J.; Williams, G. A. Studies of technetium complexes. Part 6 . The preparation, characterisation, and electron spin resonance spectra of salts of tetrachloro- and tetrabromo-nitridotechnetate(VI): crystal structure of tetraphenylarsonium tetrachloronitridotechnetate(VI). J. Chem. Soc., Dalton Trans. 1984, 2395-2400.

(217) Kersting, M.; Friebel, C.; Dehnicke, K.; Krestel, M.; Allmann, R. Synthese, IR- und EPR-Spektren sowie die Kristallstruktur von $\mathrm{HPPh}_{3}\left[\mathrm{WOCl}_{4}\left(\mathrm{OPPh}_{3}\right)\right]$. Z. Anorg. Allg. Chem. 1988, 563, 70-78.

(218) Voigt, A.; Abram, U.; Kirmse, R. The existence of $\left[\mathrm{ReNF}_{4}\right]^{-}-$an EPR study. Inorg. Chem. Commun. 1998, 1, 141-142.

(219) Voigt, A.; Abram, U.; Böttcher, R.; Richter, U.; Reinhold, J.; Kirmse, R. Q-Band single-crystal EPR study and molecular orbital calculations of $\left[\left(\mathrm{C}_{6} \mathrm{H}_{5}\right)_{4} \mathrm{As}\right]\left[\mathrm{Re}^{\mathrm{VI}} \mathrm{NCl}_{4} / \mathrm{Re}^{\mathrm{V}} \mathrm{OCl}_{4}\right]$. Chem. Phys. 2000, 253, 171-181.

(220) Abram, U.; Braun, M.; Abram, S.; Kirmse, R.; Voigt, A. $\left[\mathrm{NBu}_{4}\right]\left[\mathrm{ReNCl}_{4}\right]$ : Facile synthesis, structure, electron paramagnetic resonance spectroscopy and reactions. $J$. Chem. Soc., Dalton Trans. 1998, 231-238.

(221) Borisova, L. V.; Ermakov, A. N.; Plastinina, Y. I.; Prasolova, O. D.; Marov, I. N. Determination of rhenium based on the formation of rhenium(VI) oxide halide complexes by spectrophotometry and electron spin resonance spectroscopy. Analyst 1982, 10\%, $500-504$.

(222) Holloway, J. H.; Hope, E. G.; Raynor, J. B.; Townson, P. T. Magnetic resonance studies on osmium pentafluoride oxide. J. Chem. Soc., Dalton Trans. 1992, 1131-1134. 
(223) Manual of TURBOMOLE V7.5.1 2021, a development of University of Karlsruhe and Forschungszentrum Karlsruhe GmbH, 1989-2007, TURBOMOLE GmbH, since 2007; available from https://www.turbomole.org/turbomole/ turbomole-documentation/(retrieved August 28, 2021).

(224) Dyall, K. G. Relativistic double-zeta, triple-zeta, and quadruple-zeta basis sets for the 6d elements Rf-Cn. Theor. Chem. Acc. 2011, 129, 603-613.

(225) Luber, S.; Malkin Ondík, I.; Reiher, M. Electromagnetic fields in relativistic oneparticle equations. Chem. Phys. 2009, 356, 205-218.

(226) Weltner Jr., W. Magnetic Atoms and Molecules; Van Nostrand Reinhold: New York, USA, 1983.

(227) Holzer, C.; Franzke, Y. J.; Kehry, M. Assessing the Accuracy of Local Hybrid Density Functional Approximations for Molecular Response Properties. J. Chem. Theory Comput. 2021, 17, 2928-2947.

(228) Lehtola, S.; Dimitrova, M.; Fliegl, H.; Sundholm, D. Benchmarking Magnetizabilities with Recent Density Functionals. J. Chem. Theory Comput. 2021, 17, 1457-1468.

(229) Lehtola, S.; Dimitrova, M.; Fliegl, H.; Sundholm, D. Correction to "Benchmarking Magnetizabilities with Recent Density Functionals". J. Chem. Theory Comput. 2021, 17, 1457-1468.

(230) Teale, A. M.; Lutnæs, O. B.; Helgaker, T.; Tozer, D. J.; Gauss, J. Benchmarking density-functional theory calculations of NMR shielding constants and spin-rotation constants using accurate coupled-cluster calculations. J. Chem. Phys. 2013, 138, 024111.

(231) Hedegård, E. D.; Kongsted, J.; Sauer, S. P. A. Validating and Analyzing EPR Hyper- 
fine Coupling Constants with Density Functional Theory. J. Chem. Theory Comput. 2013, 9, 2380-2388.

(232) Flaig, D.; Maurer, M.; Hanni, M.; Braunger, K.; Kick, L.; Thubauville, M.; Ochsenfeld, C. Benchmarking Hydrogen and Carbon NMR Chemical Shifts at HF, DFT, and MP2 Levels. J. Chem. Theory Comput. 2014, 10, 572-578.

(233) Stoychev, G. L.; Auer, A. A.; Izsák, R.; Neese, F. Self-Consistent Field Calculation of Nuclear Magnetic Resonance Chemical Shielding Constants Using Gauge-Including Atomic Orbitals and Approximate Two-Electron Integrals. J. Chem. Theory Comput. 2018, 14, 619-637.

(234) Lutnæs, O. B.; Teale, A. M.; Helgaker, T.; Tozer, D. J.; Ruud, K.; Gauss, J. Benchmarking density-functional-theory calculations of rotational $g$ tensors and magnetizabilities using accurate coupled-cluster calculations. J. Chem. Phys. 2009, 131, 144104.

(235) Alonso, P.; Alcalá, R.; Usón, R.; Forniés, J. EPR study of mononuclear Pt(III) organometallic complexes. J. Phys. Chem. Solids 1991, 52, 975-978.

(236) Komorovský, S.; Repiský, M.; Ruud, K.; Malkina, O. L.; Malkin, V. G. FourComponent Relativistic Density Functional Theory Calculations of NMR Shielding Tensors for Paramagnetic Systems. J. Phys. Chem. A 2013, 117, 14209-14219.

(237) Ramsey, N. F. Magnetic Shielding of Nuclei in Molecules. Phys. Rev. 1950, 78, 699703.

(238) Ramsey, N. F. Dependence of Magnetic Shielding of Nuclei upon Molecular Orientation. Phys. Rev. 1951, 83, 540-541. 